University of Louisville ThinkIR: The University of Louisville's Institutional Repository

Electronic Theses and Dissertations

$8-2016$

\title{
In vivo monitoring of therapeutic efficacy and virulence profile by bioluminescent Klebsiella pneumoniae.
}

Ramy Ameen Fodah

University of Louisville

Follow this and additional works at: https://ir.library.louisville.edu/etd

Part of the Medical Immunology Commons, and the Medical Microbiology Commons

\section{Recommended Citation}

Fodah, Ramy Ameen, "In vivo monitoring of therapeutic efficacy and virulence profile by bioluminescent Klebsiella pneumoniae." (2016). Electronic Theses and Dissertations. Paper 2542.

https://doi.org/10.18297/etd/2542

This Doctoral Dissertation is brought to you for free and open access by ThinkIR: The University of Louisville's Institutional Repository. It has been accepted for inclusion in Electronic Theses and Dissertations by an authorized administrator of ThinkIR: The University of Louisville's Institutional Repository. This title appears here courtesy of the author, who has retained all other copyrights. For more information, please contact thinkir@louisville.edu. 


\title{
IN VIVO MONITORING OF THERAPEUTIC EFFICACY AND VIRULENCE PROFILE BY BIOLUMINESCENT KLEBSIELLA PNEUMONIAE \\ By:

\author{
Ramy Ameen Fodah
}

B.S. King Saud University, 2005

M.S. University of Louisville, 2012

\author{
A Dissertation \\ Submitted to the Faculty of the \\ School of Medicine of the University of Louisville \\ in Partial Fulfillment of the Requirements \\ for the Degree of
}

Doctor of Philosophy in Microbiology and Immunology

\author{
Department of Microbiology and Immunology \\ University of Louisville \\ Louisville, Kentucky
}

August 2016 
Copyright 2016 by Ramy Ameen Fodah

All rights reserved 

IN VIVO MONITORING OF THERAPEUTIC EFFICACY AND VIRULENCE

PROFILE BY BIOLUMINESCENT KLEBSIELLA PNEUMONIAE

By:

Ramy Ameen Fodah

B.S. King Saud University, 2005

M.S. University of Louisville, 2012

A Dissertation Approved on:

June 13, 2016

by the following Dissertation Committee:

Dr. Jonathan Warawa (Chair)

Dr. Matthew B. Lawrenz

Dr. James E. Graham

Dr. Michele M. Kosiewicz

Dr. David A. Scott 


\section{DEDICATION}

I would like to dedicate this dissertation to my mother Fatheyah, my father Ameen, my wife Nouf, my son Ahmed, my brothers Ibraheem and Abdulhameed, and to my friends. Your tremendous and continuous support is what made my success in my graduate program possible. 


\section{ACKNOWLEDGEMENTS}

All praises belongs to Almighty Allah, the most beneficent, gracious, and merciful for giving me the opportunity, resolution, and capacity to carry out and complete this dissertation.

My deepest gratitude for Dr. Warawa for his continuous support and valuable advises both on the personal and professional levels. I believe that without his involved teaching methods and constructive instructions none of my projects would have come to see the light.

I would like to extend my appreciation to my committee members Dr.

Matthew B. Lawrenz, Dr. David A. Scott, Dr. Michele M. Kosiewicz, and Dr. James E. Graham for their priceless inputs which helped, guided, and refined my progress in my research projects. Their words of encouragement and support have nourished my growth in my graduate program.

I also would like to thank my dear colleagues, the past members of the Warawa laboratory, Jacob Scott, Maria Gutierrez, and Tia Pfeffer whom I had a great pleasure to work with. Tia Pfeffer help me greatly as a friend that I go back to for personal advises and she was a tremendous help for all lab members by preparing and maintaining the supplies needed for conducting our work. Jacob and Maria are great friends that made the time that I have spent in the Warawa laboratory more enjoyable. 
My precious wife Nouf and my dear son Ahemd were the greatest emotional support that I could have ever asked for that made my success in my graduate program possible. Being from a different country, Nouf has been the only family that I have in the United States and she has done a wonderful job supporting me during my graduate study. In addition to being a great wife and friend, Nouf is a wonderful mother to our son Ahmed. My lovely son Ahmed was a blissed addition to our family whom his pleasant laughter and sweet smiles always brings happiness that helped me and my wife survive through any hardships we came across. Thank you Nouf and Ahmed for being part of my life! I would like to send my thanks overseas to the family and friends I have in Saudi Arabia for their motivating words and for their nonstop prayers. My loving parents have always been a great support both emotionally and financially.

I would like to thank the Department of Microbiology and Immunology at University of Louisville for giving me the opportunity to be part of this prestigious and successful institution. It is a lovely place where all members of the department are like one big family. I also would like to thank University of Louisville, King Saud bin Abdulaziz University for Health Sciences (KSAU-HS), and the Government of Saudi Arabia for their sponsorship and funding that allowed me to pursue my higher education and conduct my research projects. 


\begin{abstract}
IN VIVO MONITORING OF THERAPEUTIC EFFICACY AND VIRULENCE

PROFILE BY BIOLUMINESCENT KLEBSIELLA PNEUMONIAE

Ramy Ameen Fodah

June 13, 2016
\end{abstract}

Klebsiella pneumoniae causes an acute respiratory infection in human with severe outcomes and high mortality rates even with antibiotic treatment. Even with its critical clinical importance, few virulence systems have been identified for K. pneumoniae limiting the development of new therapeutic strategies. Accordingly, we performed Next Generation sequencing for the strain ATCC 43816, a virulent strain in mouse respiratory disease models, and compared its genomic data with two previously sequenced strains NTUH-K2044 and MGH 78578 for the purpose of identifying genes required for colonizing host lungs. Furthermore, the virulence potential of the three K. pneumoniae strains were tested in a mouse model of pulmonary disease uniquely generated by our group to insure the specific delivery of an inoculum into host lungs allowing for studying diseases associated specifically with the lower respiratory tract. To monitor disease progression noninvasively, a bioluminescent $K$. pneumoniae strain was engineered which allowed for monitoring meropenem therapeutic efficacy against the bacteria in real time. A transposon mutant library was 
generated in the bioluminescent strain and introduced into mice lungs in order identify critical fitness factors required by $K$. pneumoniae to survive the selective pressure of host lung. The attenuation of known and potential virulence factors, including capsular polysaccharide (CPS) and type 6 secretion systems (T6SSs), were tested in our lung-specific murine model of respiratory disease. Similar to previous findings, manC capsule mutant was attenuated in our lung-specific disease model whereas for the vgrG T6SSs mutants, only cluster one illustrated some potential attenuation in the host, and future studies will be conducted to confirm these outcomes. K. pneumoniae is thought to be an extracellular pathogen but we have provided the first evidence suggesting that this dogma might not be entirely true by demonstrating the capability of the bacteria to proliferate within cultured macrophages in addition to the ability of a subpopulation of $K$. pneumoniae to become intracellular within mice lungs. Further studies will need to be conducted to identify the role(s) of the intracellular lifestyle for $K$. pneumoniae during the pulmonary disease. 
TABLE OF CONTENTS

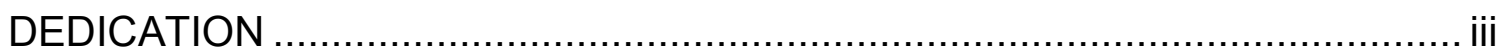

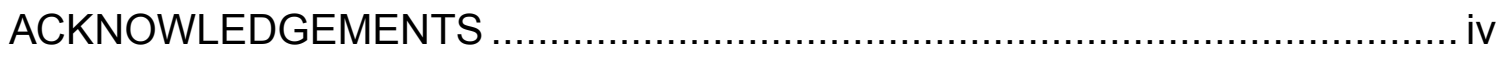

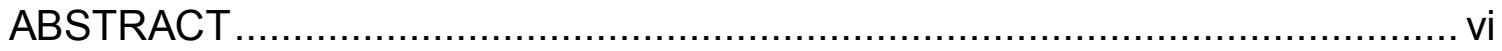

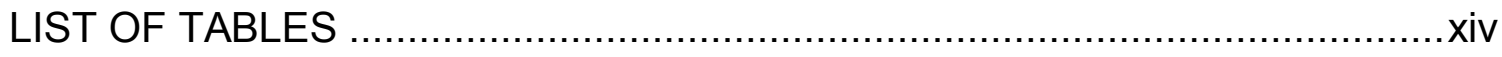

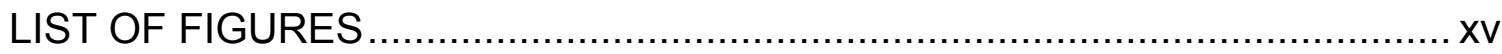

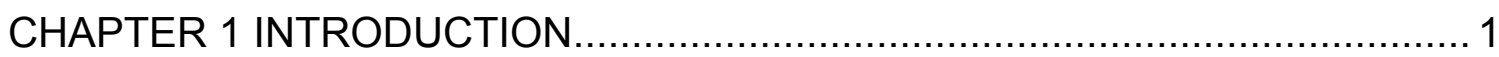

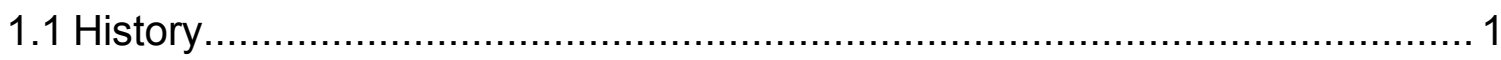

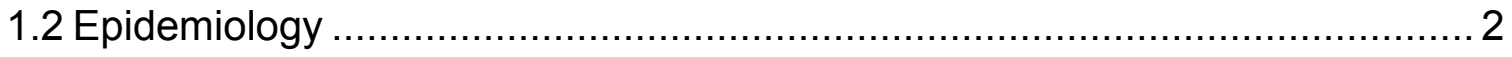

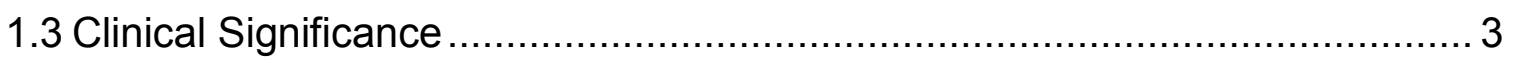

1.3.1 K. pneumoniae respiratory infections .............................................. 4

1.3.1.1 Hospital-acquired pneumonia .................................................. 4

1.3.1.2 Community-acquired pneumonia ............................................... 4

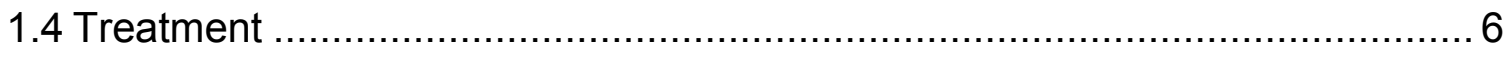

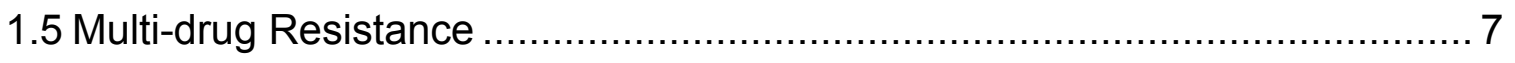

1.5.1 Extended-Spectrum Beta-Lactamases ............................................... 8

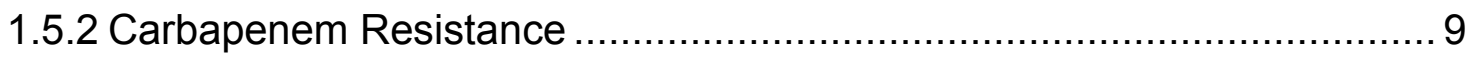

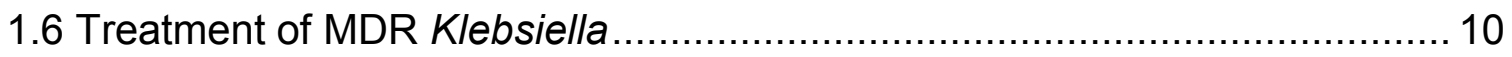


1.6.1 Treatment of ESBL-producing Klebsiella ......................................... 10

1.6.2 Treatment of carbapenem resistant Klebsiella ................................... 11

1.7 K. pneumoniae murine respiratory infection models ................................ 12

1.7.1 Host response to K. pneumoniae pneumonia ................................... 13

1.7.2 Therapeutic K. pneumoniae lung infection models ............................. 16

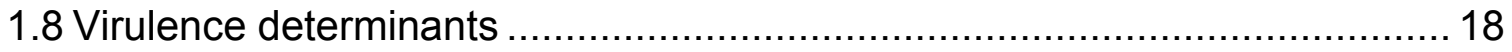

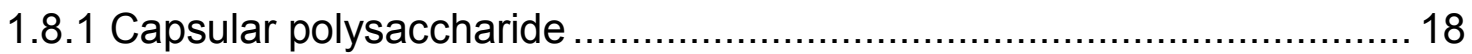

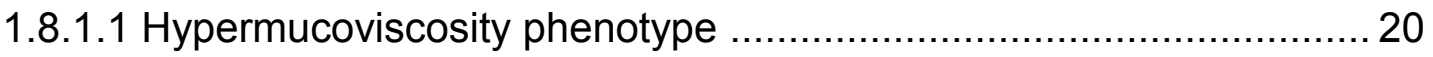

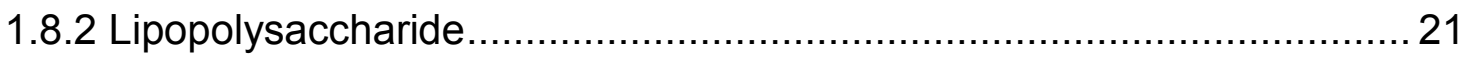

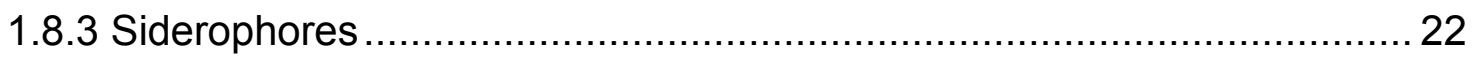

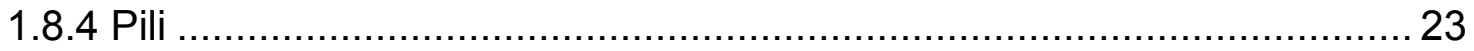

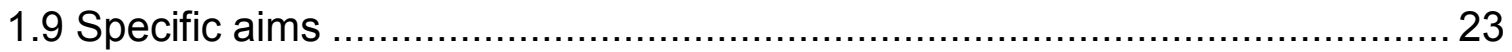

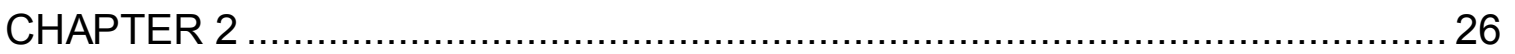

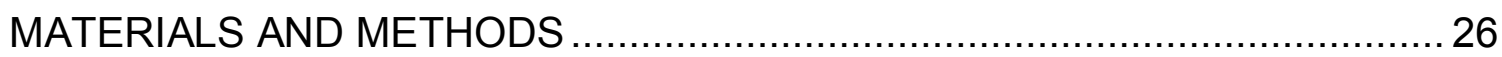

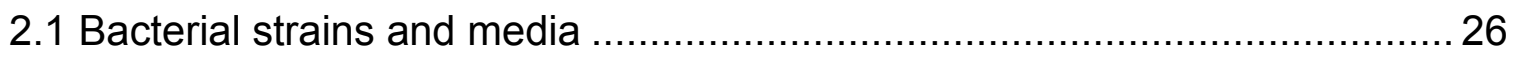

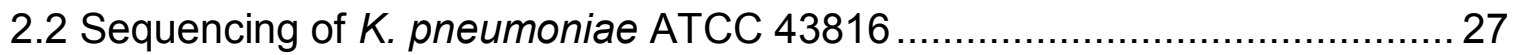

2.3 Quantification of capsular polysaccharide production............................... 28

2.4 Generation of capsular polysaccharide mutants ................................... 28

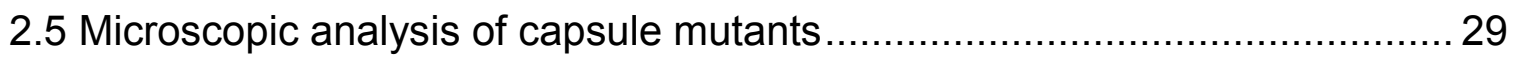

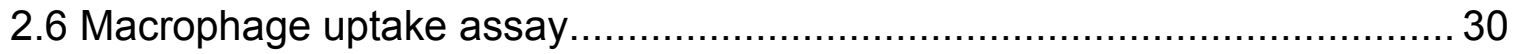


2.7 Macrophage survival assay

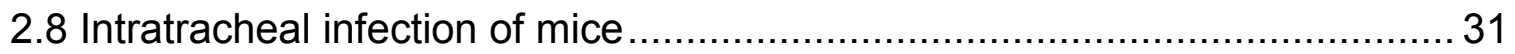

2.9 Bacterial enumeration from key sites of infection ................................... 32

2.10 Creating the bioluminescent JSKP001 strain ........................................ 32

2.11 In vitro proliferation of JSKP001 and ATCC 43816 ................................. 33

2.12 In vitro correlation of JSKP001 bioluminescence and bacterial numbers ... 34

2.13 In vivo fitness and real-time monitoring of $K$. pneumoniae in mice ............. 34

2.14 Measuring the correlation between bioluminescence and bacterial numbers

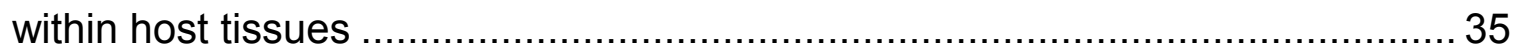

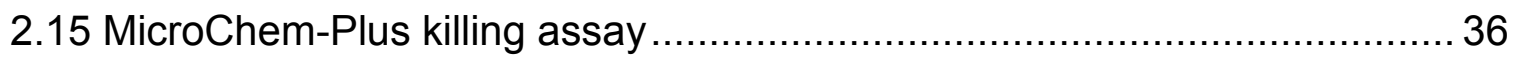

2.16 In vitro bacterial growth inhibition by meropenem.................................. 36

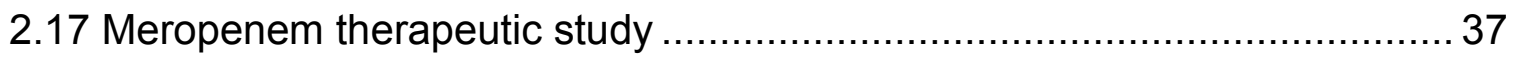

2.18 Creating the bioluminescent man $C$ and $v g r G$ mutants ......................... 37

2.19 Testing the fitness of the JSKP001 $\Delta$ manC in vivo.................................. 38

2.20 Monitoring JSKP001 $\Delta$ manC early clearance in vivo................................ 39

2.21 Tracking JSKP001 replication within cultured macrophages .................... 39

2.22 Creating bioluminescent $K$. pneumoniae strains with different promoters .. 40

2.23 Monitoring the bioluminescent $K$. pneumoniae strains replication within

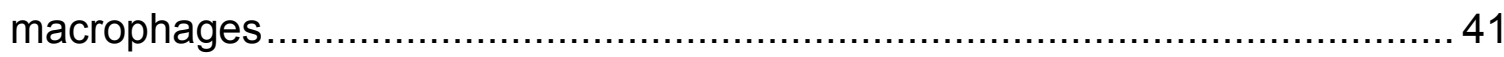

2.24 Estimation of intracellular bacteria within host lungs ............................. 41 
2.25 Tn-seq study.

2.25.1 Creating transposon library in JSKP001

2.25.2 Infecting albino C57BL/6J mice with the generated Tn-seq library....... 44

2.26 Testing the virulence of the $\operatorname{vgrG}$ mutants in single strain infection model . 45

2.27 Testing vgrG mutants fitness using in vivo competition study.................. 45

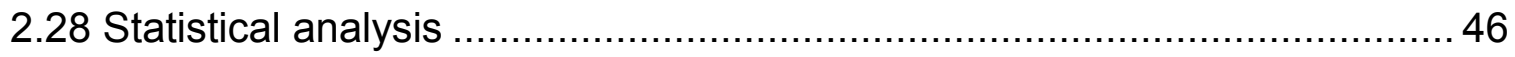

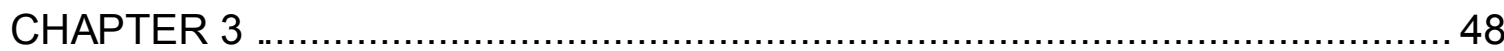

CORRELATION OF KLEBSIELLA PNEUMONIAE COMPARATIVE GENETIC ANALYSES WITH VIRULENCE PROFILES IN A MURINE RESPIRATORY

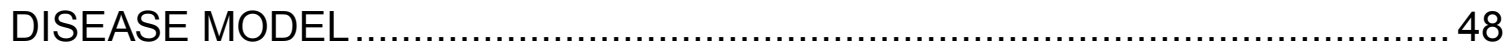

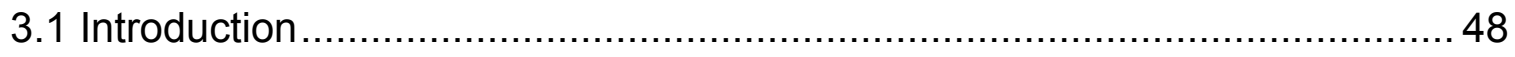

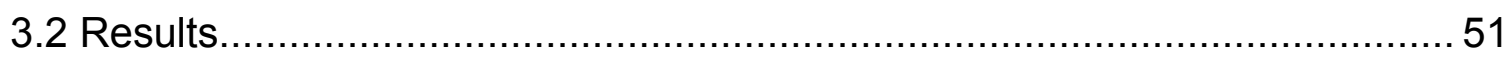

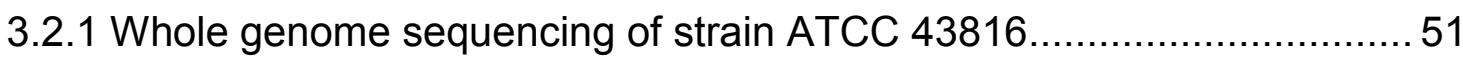

3.2.2 Characterization of capsule production ........................................... 53

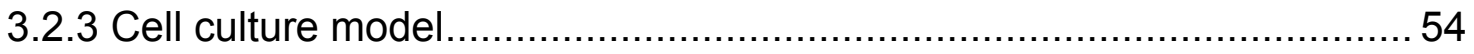

3.2.4 Respiratory murine model of $K$. pneumoniae infection ........................ 57

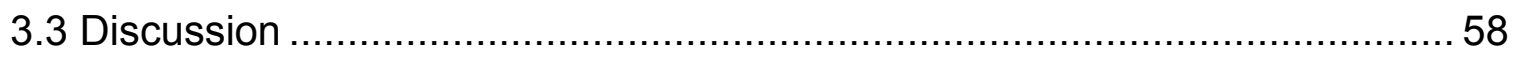

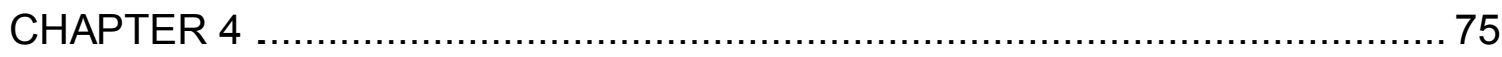

DIRECT MONITORING OF MEROPENEM THERAPEUTIC EFFICACY AGAINST KLEBSIELLA PNEUMONIAE RESPIRATORY INFECTION USING

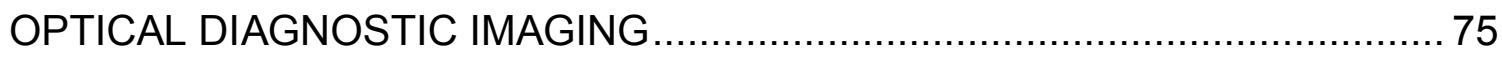




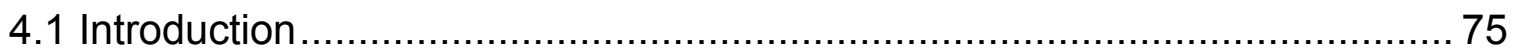

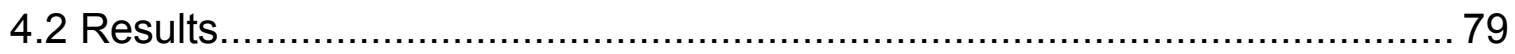

4.2.1 Generation of bioluminescent $K$. pneumoniae bioreporter strain ............79

4.2.2 Noninvasive tracking of respiratory disease .................................. 81

4.2.3 Bioluminescence correlates with bacterial burdens ........................... 84

4.2.4 In vitro monitoring of bacterial viability using bioluminescence ............. 85

4.2.5 In vitro tracking of bacterial growth inhibition by meropenem ................ 86

4.2.6 Noninvasive monitoring of meropenem therapeutic efficacy in mice ...... 87

4.2.7 Tracking the clearance of manC mutant using optical diagnostic

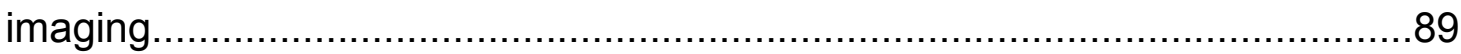

4.2.8 Monitoring intracellular growth of K. pneumoniae.............................. 92

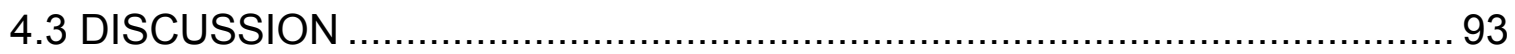

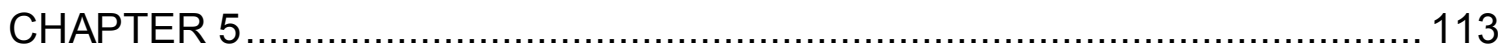

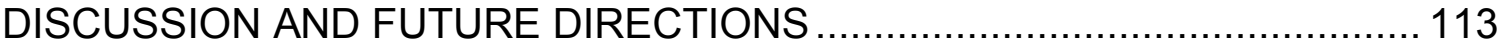

5.1 Identifying virulence factors required by $K$. pneumoniae to establish the

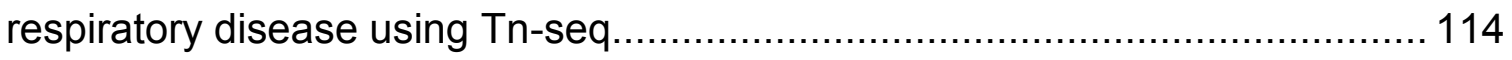

5.2 The role of T6SS for K. pneumoniae in disease .................................. 117

5.3 The presentation of pulmonary disease using IMIT ............................... 126

5.4 The importance of intracellular life style for K. pneumoniae pathogenesis . 127

5.5 Future implications for the bioluminescent K. pneumoniae 129 
REFERENCES

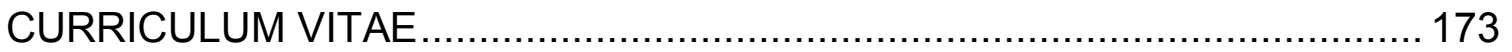




\section{LIST OF TABLES}

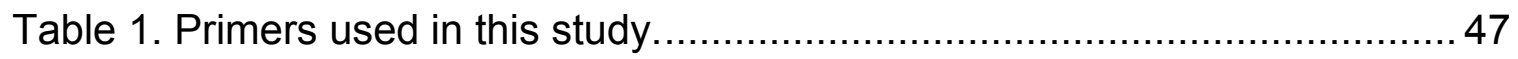

Table 2. Genetic elements common/unique to sequenced strains ...................65

Table 3. Probit analysis of IMIT-infections of BALB/c mouse using $K$.

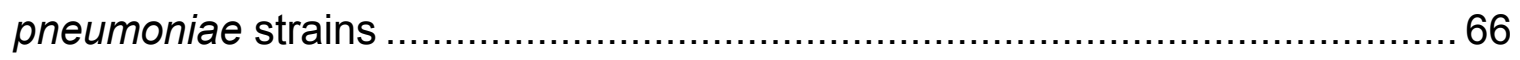




\section{LIST OF FIGURES}

Figure 1. Gentamicin killing of K. pneumoniae at room temperature

Figure 2. Alignment of the ATCC 43816 sequence to previously sequenced $K$.

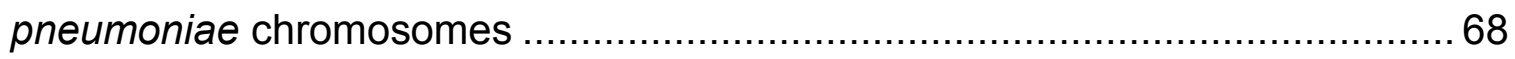

Figure 3. Genetic organization of the ATCC 43816 K2 capsule locus ................ 69 Figure 4. Negative staining of capsular polysaccharide from ATCC 43816 and

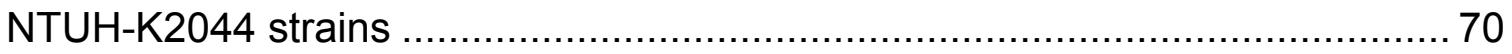
Figure 5. Uptake of K. pneumoniae wild type and capsular polysaccharide mutants strains into cultured murine macrophage ....................................... 71 Figure 6. Growth potential of K. pneumoniae strains in cultured murine

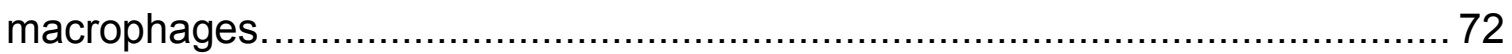

Figure 7. Survival analysis of $K$. pneumoniae respiratory challenge ................. 73

Figure 8. Bacterial burden of K. pneumoniae-infected mice ............................ 74

Figure 9. Growth curves of JSKP001 and ATCC 43816 strains ...................... 98

Figure 10. Survival analysis and bacterial systemic dissemination.................... 99

Figure 11. JSKP001 LD50 determination in albino C57BL/6J mice .................. 100

Figure 12. In vivo tracking of bacterial proliferation ..................................... 101

Figure 13. Correlation of bioluminescence and bacterial burdens in host tissues 103

Figure 14. Tracking bacterial viability upon MicroChem-Plus treatment using bioluminescence 104 
Figure 15. Monitoring the impact of meropenem treatment on bacterial growth using bioluminescence 105

Figure 16. In vivo monitoring of meropenem efficacy against $K$. pneumoniae respiratory infection in mice 107

Figure 17. Noninvasive monitoring of JSKP001 $\Delta$ manC fitness in mice 108

Figure 18. Tracking the clearance of the capsule mutant using bioluminescence imaging 109

Figure 19. Monitoring bacterial replication within cultured macrophages 110 Figure 20. Tracking K. pneumoniae proliferation within cultured macrophages using different promoter systems

Figure 21. Estimating bacterial internalization into host cells within mice lung. 112 Figure 22. Noninvasive monitoring of JSKP001 Tn-seq library in lung-specific disease model 116

Figure 23. In vivo fitness of $K$. pneumoniae T6SS mutants 119

Figure 24. Fitness of the vgrG mutants in competition to the wild type strain ... 122 Figure 25. Proposed model of the indirect contribution of T6SS in K. pneumoniae nosocomial respiratory disease 125 


\section{CHAPTER 1 \\ INTRODUCTION}

\subsection{History}

The Klebsiella genus was named after the German microbiologist Edwin Klebs whom due to his efforts in combination with other researchers we came to understand the ability of this bacteria in causing acute respiratory infections in humans [1-3]. The first isolation of bacteria from the Klebsiella species was from patients with rhinoscleroma, a chronic granulomatous disease of the upper respiratory tract [4], which was named by Trevisan at 1887 as Klebsiella rhinoscleromatis. At 1893, Abel observed capsulated rod-shaped organisms in nasal secretion of patients with ozaena, and therefore, he named that bacteria as Bacillus mucosus ozaenae which was later transferred into the Klebsiella genus as Klebsiella ozaenae [5].

Among the Klebsiella genus, Klebsiella pneumoniae is the most clinically significant species which is also known as Friedländer's bacillus due to the effort done by his group where Hans Christian Gram developed the Gram staining that enabled distinguishing Klebsiella pneumoniae from Streptococcus pneumoniae as causative agents for respiratory disease in humans $[3,6]$. Initially, Klebsiella rhinoscleromatis and Klebsiella ozaenae were recognized as distinct species, but 
due to their indistinguishable DNA relatedness to Klebsiella pneumoniae [7], they are now considered as a subspecies of Klebsiella pneumoniae according to Ørskov's Bergey's Manual of Systematic Bacteriology [8]. For the purpose of this dissertation Klebsiella pneumoniae subspecies pneumoniae will be referred to as K. pneumoniae.

\subsection{Epidemiology}

Klebsiella species are ubiquitous in the environment in which they are found in soil, sewage, surface water, and on plants [9-13]. They are also good colonizers of mammalian mucosal surfaces where they colonize human, swine, and equine [14]. K. pneumoniae is a saprophyte in human's nasopharynx and gastrointestinal $(\mathrm{Gl})$ tract where the asymptomatic carriage rate of the bacteria varies from $1-6 \%$ in the nasopharynx, with increased carrier rate with alcoholism, and from $5-38 \%$ in stool samples $[14,15]$. Klebsiella spp. is rarely found on the skin where it is considered to be a transient flora [16].

In the hospital setting, the carrier rate of Klebsiella increases significantly in patients as well as in health care personnel [17-19]. The carrier rate of the bacteria in patients is proportionally related to length of hospitalization and the excessive use of antibiotics, especially those with broad-spectrum effect [20, 21]. Patients with hospital-acquired intestinal Klebsiella have four-fold higher rate of Klebsiella nosocomial infections than non-carriers [22]. With such knowledge in mind, prescription and administration of antibiotics must be wisely controlled by 
health care providers in addition to the application of high hygienic practices to prevent the spread of the bacteria in the hospital.

The main mode of transmission of Klebsiella in the hospital is through the fecal-oral route and the contaminated hands of the health care providers [23]. The rapid spread capability of the bacteria lead to outbreaks in the hospital setting especially in the neonatal wards [24, 25]. Klebsiella have caused 13 epidemic hospital-acquired infections out of 145 epidemics between 1983 and $1991[14,26]$; bacteria from this species is responsible for $8 \%$ of endemic nosocomial infections and $3 \%$ of epidemic outbreaks according to the CDC [27]. The ability of Klebsiella to cause outbreaks in the hospital in addition to the increase in the multidrug resistant (MDR) isolates makes this bacterium a public threat to human health.

\subsection{Clinical Significance}

K. pneumoniae is mainly a nosocomial pathogen where it causes urinary tract infections, septicemia, wound infections, intestinal infections, and pneumonia in susceptible individuals [14]. People at risk of acquiring $K$. pneumoniae infection include immunocompromised individuals such as neonates and the elderly [14]. People with underlying diseases or undergoing treatments that impair their immune defenses are also predisposed to $K$. pneumoniae infections. This include patients with chronic obstructive pulmonary disease (COPD), diabetes mellitus, hepatobiliary disease, malignancies, renal failure, alcoholism, and those undergoing glucocorticoid therapy [28-38]. Among the 
other infections, the respiratory disease caused by $K$. pneumoniae is very severe and is associated with high mortality rates even with antibiotic administration [14].

\subsubsection{K. pneumoniae respiratory infections}

\subsubsection{Hospital-acquired pneumonia}

K. pneumoniae is a common causative agent for respiratory disease in the hospital in which it is responsible for $8.4 \%$ of ventilator-associated pneumonia (VAP), $7.1 \%$ of hospital-acquired pneumonia (HAP), and $7.6 \%$ of non-nosocomial healthcare-associated pneumonia (HCAP), which involves infections that take place in long-term care facilities [39]. The clinical picture of HAP or VAP by $K$. pneumoniae involves pulmonary infiltrate, fever, cough with increased sputum production, and leukocytosis $[40,41]$. Upper respiratory tract bacterial colonization in hospitalized patients is common, especially in those who require ventilator support or patients that need to be admitted into the ICU [41]. K. pneumoniae HAP often present as bronchitis or bronchopneumonia and the most frequent computed tomography (CT) scan observations include ground glass opacities, alveolar consolidation, intralobar reticular opacities, and pleural effusions [42].

\subsubsection{Community-acquired pneumonia}

K. pneumoniae is an uncommon causative agent for community-acquired pneumonia (CAP) in the USA, Europe, Argentina, and Australia where it mostly affect diabetic, alcoholic patients, or individuals with severe COPD [28, 43]. 
However, more cases of $K$. pneumoniae CAP have been reported in Taiwan and South Africa, which might be due to differences in socioeconomic factors such as primary healthcare and environmental sanitation, bacterial strains, or host defense $[28,44]$. Another possibility includes differences in characterizing the respiratory disease caused by K. pneumoniae as CAP since in some studies HCAP cases are included as part of the CAP [45]. HCAP includes hospitalization for a couple of days during the preceding three months, residence in nursing home or extended care facility, home wound care, chronic dialysis during the past month, and home infusion therapy [45]. Thus, the cases that are being reported as K. pneumoniae CAP in such studies could actually be acquired from healthcare settings rather than from the community. Although $K$. pneumoniae is not a frequent causative agent for CAP, this bacterium accounts for a higher fraction of isolates associated with more critical infections [39, 46-48].

The clinical manifestation of CAP by K. pneumoniae resemble the pneumonic disease acquired from the hospital setting, which include fever, coughing, increased sputum secretion, pleuritic chest pain, tachypnea, dyspnea, crackles during the physical examination, and leukocytosis [41]. Furthermore, $K$. pneumoniae CAP is also accompanied with significant inflammation and necrosis that progress into thick, mucoid, and blood-tinged sputum which is alluded to as "currant jelly" sputum [41]. Unlike to the radiographic observations in $K$. pneumoniae HAP, the community-acquired disease frequently generate a lobar respiratory infection similar to the radiological picture of pneumonia caused by Streptococcus pneumoniae $[42,49]$; therefore, the radiographic findings alone 
are not enough to identify Klebsiella as the causative agent for the pulmonary disease.

Mortality rates of $K$. pneumoniae CAP are quite high, even with the early administration of proper antibiotic regimen, especially if the bacteria disseminated to the bloodstream causing bacteremia $[41,50]$. In patients with lobar pneumonia, respiratory failure is the most common cause of death, especially when the bacteria disseminate rapidly, leading to multi-lobar involvement or if the pneumonic disease progressed into an acute stage [41]. Therefore, the rapid administration of the proper antibiotic therapy is of a great importance for preventing the development of a lethal incurable infection.

\subsection{Treatment}

The empiric treatment for patients with severe K. pneumoniae respiratory infection include antibiotics effective against Gram-negative bacteria (GNB), aggressive ventilation, and clinical and radiographic monitoring for entities treatable through surgical intervention such as lung abscess, pulmonary gangrene, and empyema [51-53]. Most K. pneumoniae respiratory infections are treatable by third generation cephalosporin or quinolones, while macrolides antibiotics are not effective against this bacteria [54]. Even though the advantageous activity of combination therapy is debatable [51,55-57], it is still being used for treating Klebsiella respiratory infections, and the recommendation is to use beta-lactam in addition to an aminoglycoside antibiotic to which the bacteria is susceptible instead of using two beta-lactam agents [54]. 
When treating a respiratory infection cause by GNB using aminoglycoside, it is critical to dose the antibiotic aggressively aiming to achieve a peak concentration/minimum inhibitory concentration ratio of 10 or more during the initial 48 hours so that maximum outcomes might be attainted [58]. In addition to the systemic administration of aminoglycosides to treat lung infection caused by GNB, endotracheal delivery of the antibiotics were utilized but even though this type of therapeutic was able to eliminate pathogens from sputum more frequently, it had no significant impact on clinical outcome [59].

While treating a bacterial infection, it is critical to administer the antimicrobial agents for the appropriate duration in order to prevent disease relapse and to avoid generating drug resistant isolates. The recommended minimum duration to treat $K$. pneumoniae respiratory infection is 10 days, which should be coupled with the performance of CT scan on patient chest, in order to remove entities that need to be treated through drainage or debridement such as in the case of abscess formation or empyema [53]. Last, it has been demonstrated in most cases that it is safe to switch therapy into oral administration of quinolone to which the bacteria is susceptible if a quick improvement to the intravenous treatment was observed $[60,61]$.

\subsection{Multi-drug Resistance}

The trend of the increasing resistance to a wide range of antibiotics is alarming for K. pneumoniae clinical isolates. The ability of the bacteria to generate extended-spectrum beta-lactamases or carbapenemases should be 
taken into consideration when initiating therapeutic regimen against $K$. pneumoniae infections.

\subsubsection{Extended-Spectrum Beta-Lactamases}

The Extended-Spectrum Beta-Lactamase (ESBL) producing microorganisms confer resistance to most beta-lactam antimicrobial agents, including monobactam, penicillin, and cephalosporin limiting the treatment options [41]. Outbreaks of ESBL-producing K. pneumoniae isolates have been described around the globe [30,62-66]. Among a multi-country 455 consecutive episodes of hospital-acquired K. pneumoniae bacteremia in the period from 1996 to 1997 , the ESBL-producing isolates accounted for $31 \%$ of all cases and $44 \%$ of the episodes that were acquired in the ICU [62]. The ESBL-producing strains identified within the same healthcare facility had similar genotype patterns indicating patient-to-patient transmission [62]. Infections by ESBL-producing $K$. pneumoniae strains are associated with increased mortality rates, and therefore, safety measures should be taken to avoid the spread of such isolates within the hospital [67]. Patients receiving multiple drugs or broad-spectrum antibiotics are at higher risk for developing MDR Klebsiella strains that are not limited to ESBLs, emphasizing the importance of the proper antimicrobial administration $[30,62$, $66,68]$. 


\subsubsection{Carbapenem Resistance}

Carbapenem-hydrolyzing beta-lactamases K. pneumoniae strains have been isolated worldwide [41]. K. pneumoniae is the most frequent bacteria to carry carbapenemase (KPC) and New Delhi metallo-beta-lactamase (NDM-1) enzymes that break all beta-lactams reducing the therapeutic efficacy of carbapenems and beta-lactamase inhibitors [41]. Prior administration of broadspectrum carbapenem and/or cephalosporin is a major risk for the development of KPC and NDM-1 strains but not essentially required [69-71]. The mortality rate associated with KPC infection can get as high as $50 \%$; thus, upon the identification of a positive KPC case, infection prevention control precautions should be followed more carefully (e.g. wearing proper protective clothing and hand hygiene practices) to avoid spreading the pathogen [72].

In addition to the reduced susceptibility to beta-lactam and carbapenems, the carbapenemase-producing strains often encode for genes that make them resistant to other antibiotics including fluoroquinolones and aminoglycosides [73, 74]. For KPC, the resistance rate to fluoroquinolones is $98 \%$, and $\sim 50 \%$ to amikacin and gentamicin [75]. Resistance to carbapenem can also take place by the loss of the outer membrane porins including OmpK35 and OmpK36 coupled with the presence of the extended-spectrum and/or AmpC beta-lactamases [76, 77]. Furthermore, the use of the carbapenem antibiotics might select for KPC strains generated by porin loss [78]. Collectively, the therapeutic options for KPC are limited and the choice of antibiotic should take into account the outcomes of antimicrobial susceptibility testing. 


\subsection{Treatment of MDR Klebsiella}

\subsubsection{Treatment of ESBL-producing Klebsiella}

ESBL-producing microbes are associated more frequently with nosocomial infections rather than the community acquired ones [54]. Risk factors associated with acquiring ESBL-producing Klebsiella infection include previous infection with such isolate, ICU hospitalization, or other wards accommodation that are known to contain endemic ESBL-producing organisms, and if the patient has a recent history of third generation cephalosporin administration [54]. The most effective treatment for the ESBL-producing isolates is the carbapenem drugs [79-81].

Imipenem and meropenem antimicrobial agents of the carbapenem family are the drugs of choice for treating ESBL-producing bacteria [79]. Although there is some in vitro data supporting the use of carbapenem and aminoglycoside combination therapy, such as the use of carbapenem with amikacin to treat severely ill patients, there is no clinical evidence that illustrate a clear advantage of such treatment over monotherapy [54]. Furthermore, quinolones are being used as a second-line treatment for $K$. pneumoniae infections caused by ESBLproducing isolates even though there is a higher possibility for the development of resistance to these antibiotics by ESBL-producing bacterial isolates than those that are not $[79,82]$.

The combination of beta-lactam/beta-lactamase inhibitor such as ticarcillin/clavulanate or piperacillin/tazobactam might be effective against ESBLproducing $K$. pneumoniae but they are subjected to higher minimum inhibitory 
concentrations (MICs) as the bacterial inoculum rises [83], and treatment failures have been observed with these antibiotic even with the clear in vitro susceptibility $[84,85]$. Similarly, the third and fourth generation cephalosporins MICs become higher as the inoculum of the infecting organism increases even with the apparent in vitro activity against ESBL-producing microbes [86, 87]. Clinical data demonstrated that failure rates of cephalosporins are unacceptably high when used in treating ESBL-producing organisms [88]; therefore, the Clinical and Laboratory Standards Institute (CLSI) recommends that all ESBL-producing Klebsiella isolates to be regarded as resistant to all cephalosporins [54].

\subsubsection{Treatment of carbapenem resistant Klebsiella}

Even with the critical clinical significance of the carbapenem resistant GNB, currently there are no optimal therapeutic approaches available against those microorganisms. The reason behind such limitation is the lack of large controlled clinical trials testing the effectiveness of available antimicrobial agents against diseases caused by the carbapenem resistant bacteria. The recommended therapeutic data is generated from small case reports and retrospective studies $[89,90]$. Empiric treatment against KPC involves the use of high dose of carbapenem such as meropenem or doripenem in combination with polymyxin $B$, and continuous infusion of the carbapenem drugs was found to be superior to single dose injections [91].

In addition, preferable outcomes can be achieved upon using combination therapy with a third antibiotic that can be added to the empiric treatment including 
tigecycline, aminoglycoside, fosfomycin, or rifampin [91]. If the bacteria is resistant to all aforementioned therapeutic options, then polymyxin $\mathrm{B} /$ colistin treatment can be used as long as the MIC against the invading pathogen is $\leq 2$ $\mu \mathrm{g} / \mathrm{ml}$ [91]; other antibiotic options can be utilized after relaying on the outcomes of the in vitro susceptibility testing. Due to the continuously emerging MDR bacterial isolates, new therapeutic strategies should always be investigated and model systems that allow for testing those treatment approaches should be developed.

\subsection{K. pneumoniae murine respiratory infection models}

Surrogate animal models were used in biomedical research to answer many questions that scientists could not address using in vitro model systems. They provided valuable information about the in vivo efficacy of antimicrobial agents, host immune response to invading bacteria, and virulence factors required by a pathogen to cause disease. $K$. pneumoniae is capable of colonizing multiple anatomical sites within a host; therefore, several surrogate animal models have been developed for this critical pathogen including urinary tract, GI tract, systemic, intramuscular, and respiratory infection models [92-98].

K. pneumoniae pulmonary infection can be induced in mice through different methods of bacterial inoculation including aerosolization, intranasal, surgical intratracheal, nonsurgical intratracheal, and retropharyngeal [99-104]. Although intranasal and retropharyngeal infection models are easier to perform than intratracheal instillation, these models have some caveats including the 
possibility of colonizing host upper respiratory tract and bacterial trafficking into the GI system limiting investigations directed toward studying targeted colonization of host lungs. On the other hand, surgical intratracheal inoculation expose the animals to trauma with a possibility of surgical site infection making the nonsurgical intratracheal infection model probably the best choice for studying lung-specific disease by respiratory pathogens including $K$.

pneumoniae. Furthermore, direct-lung instillation of bacteria is a close mimic of

the typical route of entry for $K$. pneumoniae into the human host on contaminated endotracheal tubes, giving rise to VAP. Mouse model of K. pneumoniae respiratory infection recapitulate human disease in which histopathological examination following K. pneumoniae inoculation whether through intranasal or intratracheal routes revealed that the respiratory disease progress into classical bronchopneumonia with a significant influx of polymorphonuclear leukocytes (mainly neutrophils), which develops into lobar pneumonia and lung consolidation $[92,102,105-109]$.

\subsubsection{Host response to K. pneumoniae pneumonia}

Host lung possesses several mechanisms of innate immunity against insulting pathogens including physical barrier, mucociliary clearance of the microbes, epithelial cells immunostimulatory signaling, and activation of resident macrophages [110]. Murine models of respiratory disease allowed for the identification for the roles of the interleukins (ILs) against K. pneumoniae respiratory infection. Mast cell IL-6, an important component of the innate 
immunity, protects the host from pneumonia and sepsis, and IL-6 knockout mice are severely susceptible to respiratory and systemic diseases [111]. IL-12 and IL23 cytokines play a role in protecting the host against $K$. pneumoniae respiratory disease in which IL-12 is required for INF- $\gamma$ production, while IL-23 is needed for IL-17 expression; absence of either of these two cytokines makes the animals more susceptible to the disease [112]. IL-17 receptor (IL-17R) is important for bacterial defense against $K$. pneumoniae pulmonary disease by stimulating the expression of lung macrophage inflammatory protein (MIP)-2 and via granulocyte colony-stimulating factor (G-CSF) [113]. IL-17R knockout mice experienced a delayed recruitment of neutrophil into the lung and increased bacterial systemic dissemination [113]. Conversely, IL-10 has an adverse effect during the pulmonary infection in which it leads to worse disease outcomes and neutralizing IL-10 using antibodies improved bacterial clearance, enhanced proinflammatory cytokines expression, and increased host survival [108]. This might be due to the anti-inflammatory function of IL-10 in which high inflammatory response might actually be beneficial for the host in fighting the insulting bacteria.

Murine models of $K$. pneumoniae respiratory disease have improved our understanding of several host factors involved in innate and adaptive immunity. The innate immunity protein lipocalin 2 (Lcn2) protects the host against $K$. pneumoniae pulmonary infection by binding enterobactin disrupting the bacterial iron acquisition mechanism and Lcn2 $2^{-/-}$mice are more susceptible to the respiratory disease [114]. Furthermore, differential and combined roles of Tolllike receptors (TLRs) in K. pneumoniae pulmonary disease were identified in 
which TLR4 was found to stimulate the host immune response against low bacterial burden while TLR2 might be required later in the disease and/or in response to higher bacterial dose [115]. INF- $\gamma$ is critical for host defense against K. pneumoniae respiratory infection while the opposite is true when the microbes get into the bloodstream $[103,107]$. Murine $K$. pneumoniae respiratory infection model revealed the unexpected finding that G-CSF worsen the outcomes of the respiratory disease due to increase capsular polysaccharide production instead of fighting off the infection through the recruitment and activation of leukocytes [94]. Moreover, reduced platelet count correlated with increased mice mortality to K. pneumoniae respiratory infection which was associated with increased lung injury, bacterial loads in the mice lung, blood, liver, and spleen [109]. Overexpression of keratinocyte-derived chemokine $(\mathrm{KC})$ in vivo heightened polymorphonuclear leukocyte recruitment into the lung, improved pathogen clearance, and increased host survival against K. pneumoniae pulmonary infection [116]. Furthermore, irradiated immunocompromised B6D2F1/J mice were more susceptible to $K$. pneumoniae respiratory disease which might serve as a useful model in understanding the opportunistic nature of this pathogen in susceptible human host [102].

Murine model of lung disease have also provided some insights into host immune response against $K$. pneumoniae strains that differ in their virulence potential. Infecting C57BL/6J mice with a virulent K. pneumoniae ATCC 43816 strain induced the production of MIP-2, KC, and monocyte chemoattractant protein-1 (MCP-1) chemokines while IA565, a K. pneumoniae strain incapable of 
causing disease in mice, had a basal chemokines levels [95]. In addition, $K$. pneumoniae capsular polysaccharide (CPS) mutant had different inflammatory response than its parental wild type strain in which animals infected with the capsule mutant had higher levels of INF- $\gamma$, TNF- $\alpha$, IL-6, and MCP-1 at different time points during the course of the disease [101]. A better understanding of host-pathogen interactions during $K$. pneumoniae respiratory disease could facilitate the development of improved therapeutic strategies for treating the infection by manipulating the host immune system.

\subsubsection{Therapeutic $K$. pneumoniae lung infection models}

Murine models of $K$. pneumoniae respiratory infection have given valuable insights about the pharmacokinetics of different antibiotics enabling testing their efficacy and toxicity in animals before their application in humans. Kanamycin aerosol therapy against $K$. pneumoniae pulmonary infection, inoculated into mice lungs by aerosolization, were more effective than intramuscular injection of the antibiotic [104]. Ceftazidime treatment was more effective than cefotiam, amoxicillin-clavulanic acid, or kanamycin against $K$. pneumoniae respiratory disease caused by intranasal inoculation of the bacteria [117].

Surrogate animal models have also facilitated therapeutic studies against MDR K. pneumoniae strains. Doripenem was more effective in treating lethal lower respiratory tract infection, caused by ESBL-producing K. pneumoniae strain, than imipenem, and meropenem in which doripenem reduced inflammation, tissue damage, LPS endotoxin release, and bacterial burdens in 
mice lungs while improving cytokine production [118]. Furthermore, combinational therapy was found to be more effective than monotherapy for treating pulmonary infection caused by KPC, induced in neutropenic mouse model, where the most effective treatment was amikacin plus doripenem [119]. Murine models of $K$. pneumoniae respiratory disease were also used to monitor the possibility of using alternative therapeutic approaches such as dietary supplementation, modulation of host immune response, agents targeting bacterial surface structures, and bacterial killing by bacteriophages. Long-term feeding of mice with amla reduced bacterial colonization of mice lungs, which was coupled with a decrease in malondialdehyde levels, and an increase in phagocytic activity of immune cells and nitrite levels [120]. In addition, dietary supplementation of omega-3 polyunsaturated fatty acids reduced the severity of K. pneumoniae respiratory disease [121]. Peritoneal administration of the chemosynthetic peptide IK8L decreased inflammatory cytokines production, reduced lung injury, and decreased mortality rate in comparison to sham-treated mice [122]. Capsule lytic depolymerase enzyme improved the efficacy of gentamicin treatment of $K$. pneumoniae pulmonary infection in BALB/c mice [123]. Treating animals with bacteriophages isolated from MDR K. pneumoniae strain lowered bacterial burdens in mice lungs, reduced weight loss, and alleviated inflammatory cytokine levels [124]. The development of more innovative treatment approaches require the identification of therapeutic targets, and thus, it is of a great importance to conduct investigations directed toward 
identifying virulence factors required by the bacteria to cause the respiratory disease.

\subsection{Virulence determinants}

\subsubsection{Capsular polysaccharide}

K. pneumoniae capsular polysaccharide (CPS) is a surface structure that enclose and protect the bacteria from opsonophagocytosis and subsequent killing by host immune cells [125]. Klebsiella encode for a thick capsule composed of repeating subunits of four to six sugars (glucose, galactose, rhamnose, mannose and fucose), which is frequently coupled with uronic acids (as negatively charged components) $[14,126,127]$. Among the other virulence factors identified for K. pneumoniae, CPS is the best studied. CPS is a critical virulence factor for K. pneumoniae in which deleting that structure increases bacterial uptake by cultured macrophages and attenuates their ability to cause a respiratory disease in mice $[92,99]$.

The CPS antigens are classified into 77 serotypes in which their prevalence varies greatly in different countries [128-135]. The K2 capsular serotype is the most common worldwide [128]. K21, K2, and K55 capsular serotypes are the most common in North America and Europe, while K1 is the major serotype in liver abscess, bacteremia, and septic endophthalmitis in Taiwan and in liver abscess isolates in Korea [128-133]. Furthermore, K54 is the main serotype isolated from sputum, urine, and blood samples in Australia [134]. 
In general, K1 and K2 capsular serotypes are more associated with virulence than non-K1/K2 isolates [136]. The variation in the level of virulence noted among K. pneumoniae strains with different capsular serotypes can probably be explained by one of two main mechanisms. First, the capsule of the non-K1/K2 K. pneumoniae strains contains sugar sequences of L-rhamnosealpha-2/3-L-rhamnose or mannose-alpha-2/3-mannose that can be recognized by macrophages through either lectin or mannose receptors, which subsequently lead to the ingestion and killing of the microorganisms via lectinophagocytosis mechanism $[137,138]$. On the other hand, these mannose or rhamnose sequences are absent from $\mathrm{K} 1$ and $\mathrm{K} 2$ capsular serotypes making $K$. pneumoniae strains that possess such capsular serotypes resist uptake by macrophages, which make those strains probably more virulent to the host [139, 140].

The second possible explanation for the variation in the virulence of $K$. pneumoniae strains with different capsular serotypes involves the hypermucoviscous phenotype which is associated more frequently with the $\mathrm{K} 1 / \mathrm{K} 2$ strains than the other serotypes (see section 1.8.1.1) [131]. In addition to resisting phagocytosis by macrophages, some capsular serotypes such as $\mathrm{K} 1$, $\mathrm{K} 2$, and $\mathrm{K} 25$ have been proposed to play a role in disease by making the bacteria more antiphagocytic to neutrophils $[140,141]$. 


\subsubsection{Hypermucoviscosity phenotype}

Some K. pneumoniae strains produce a mucoviscous exopolysaccharide structure which is responsible for the hypermucoviscosity phenotype. These microbes form sticky colonies on microbiology agar media which can be tested by string formation test [142]. A string of more than 5 millimeters in length is indicative of a positive test and have been correlated with K. pneumoniae invasive infections [143]. The expression of hypermucoviscosity phenotype is mediated by rmpA and rmpA2 genes [144]. Proteins encoded by these genes are proposed to function as positive regulators that bind the 5' end for the CPS genes [144]. Increased mucoviscosity is predicted to be associated with ironlimited state, which is the case within a mammalian host [144].

Independent from the capsular polysaccharide serotypes, isolates that illustrate the hypermucoviscosity phenotype or increased capsule production are less susceptible to complement-mediated killing than those that do not produce excessive capsule $[143,145]$. Increased bacterial mucoviscosity is associated with high serum resistant and it was found to be present in $98 \%$ of isolates causing invasive infections in comparison to $17 \%$ only in non-invasive strains [143]. There is a major correlation between increased mucoviscosity and destructive tissue abscess syndrome, including abscess formation in the liver and in other anatomical sites [131, 146-150]. Furthermore, hypermucoviscosity phenotype was observed in a significantly higher numbers of isolates from abscess sites than nonabscess sites again emphasizing the fact that this phenotype is associated more with bacterial virulence and invasiveness [146]. 


\subsubsection{Lipopolysaccharide}

Lipopolysaccharide (LPS) O side chain plays a critical role in $K$. pneumoniae resistance to complement-mediated killing by preventing $\mathrm{C} 1 \mathrm{q}$ or $\mathrm{C} 3 \mathrm{~b}$ from attaching to the bacterial cell surface, protecting bacterial membrane from damage and subsequent cell death mediated by the complement deposition [151-154]. K. pneumoniae LPS mutants were susceptible to complementmediated killing, while the same was not true for the CPS which was found to play no role in serum resistance [151, 152]. Conversely, some of the $K$. pneumoniae isolates missing the LPS O side chain can still retain resistance to complement-mediated killing if the microbes were heavily encapsulated [145]. There are other mechanisms in which Klebsiella LPS may contribute in bacterial virulence that include intensifying the severity of the respiratory disease by increasing the tendency for bacteremia, and by triggering an inflammatory response, as an endotoxin, leading to sepsis syndrome and septic shock [155]. However, the role of LPS in $K$. pneumoniae murine respiratory infection models is controversial in which two studies demonstrated that the bacteria were attenuated upon LPS deletion $[155,156]$, while a third analysis showed no requirement of LPS in bacterial virulence [157]. Such inconsistent outcomes might be explained by the differences in the mutants created, where in those studies the LPS mutants were created by deleting specific genes rather than the whole LPS operon, which might results in variations in the structure and/or function of LPS. 


\subsubsection{Siderophores}

As one of the Enterobacteriaceae family, Klebsiella require iron for growth and due to the limited availability of iron in the microenvironment within the host, bacteria have developed mechanisms to compete for and scavenge this essential factor [14]. Such mechanisms involve the production of iron chelators known as siderophores that compete with host proteins such as transferrin and hemoglobin over iron by developing higher affinity for binding ferric iron $\left(\mathrm{Fe}^{3+}\right)$ [158]. K. pneumoniae encodes for four types of siderophores including enterobactin, aerobactin, yersiniabactin, and salmochelin which sequester iron for the bacteria $[114,159-166]$. However, only the production of aerobactin and yersiniabactin could be linked with bacterial virulence in murine infection models [162, 167-169]. Yersiniabactin is an important siderophore for $K$. pneumoniae that contribute in bacterial virulence during the respiratory disease by enabling the bacteria to evade the action of the innate immunity protein lipocalin 2 (Lcn2) $[114,159,170]$. On the other hand, salmochelin is a glycosylated form of enterobactin which enable the bacteria to evade Lcn2 attachment to the siderophore via steric hindrance preventing Lcn2 from neutralizing the iron acquisition function of the siderophore $[114,170]$. Even though the contribution of salmochelin in K. pneumoniae evasion of Lcn2 was demonstrated in ex vivo growth assays, the role of this siderophore for bacterial pathology is still unclear in which infecting mice with salmochelin isogenic mutant led to variable disease outcomes $[114,170]$. 


\subsubsection{Pili}

K. pneumoniae strains express two morphologically and functionally different types of pili including type 1 and type 3 [171]. Type 1 pilus is consists of heteropolymeric mannose-binding fibers present in all members of the Enterobacteriaceae family, which facilitate bacterial attachment to multiple host target cells [172]. The type 3 pili adhesion protein plays an essential role in the virulence of $K$. pneumoniae by allowing the bacteria to adhere to epithelial cells of the host including those of the respiratory, urogenital, and intestinal tracts [173], which might lead to bacterial colonization of those anatomical sites with subsequent proliferation on the host mucosal surfaces leading to clinical infections such as pneumonia or pyelonephritis. However, investigations have demonstrated that both type 1 and type 3 fimbriae are dispensable for gut and lung pathology while only type 1 fimbriae was found to play an important role in K. pneumoniae urinary tract infection in mice $[174,175]$. In addition to adherence to host cells, type 3 pili are essential for biofilm development by K. pneumoniae on inanimate surfaces and on human extracellular matrix which leads to the establishment of therapeutic-resistant biofilm on indwelling plastic devices such as urinary and intravenous catheters $[176,177]$.

\subsection{Specific aims}

K. pneumoniae is an important pathogen capable of causing severe lung disease in human. Our overarching goal is to understand the contribution of $K$. pneumoniae virulence factors in the ability of the bacteria to cause the respiratory 
disease in the host. To conduct our studies we wanted to work with a $K$. pneumoniae strain capable of causing acute lung disease in surrogate animal model resembling that of the human, and more importantly a strain that is amenable to genomic manipulation. K. pneumoniae ATCC 43816 strain has been utilized by different groups to study host response to lung infection but with no genomic data available restricting the ability to create mutants, which would limit investigations directed toward understanding the contribution of bacterial virulence factors in disease. Thus, we have decided to address this scientific gap by sequencing the ATCC 43816 strain, perform a genomic comparison with previously fully sequenced K. pneumoniae strains including NTUH-K2044, and MGH 75878, and compare the relationship between the sequencing data with the ability of these strains to cause a respiratory disease in mice. Furthermore, to limit the involvement of the upper respiratory tract during the infection, we decided to conduct our studies using a lung-specific mouse disease model established by our group. We predicted that ATCC 43816 and NTUH-K2044 will be more virulent in the mouse model than MGH 78578 since previous findings suggest that $K$. pneumoniae strains with $\mathrm{K} 1$ and $\mathrm{K} 2$ capsular serotypes are more virulent than strains with other capsular serotypes, which might be related to the ability of $\mathrm{K} 1 / \mathrm{K} 2$ strains to resist phagocytosis by host immune cells. However, $K$. pneumoniae encode for a large genome and capsule is probably not the only factor that play a role in virulence; therefore, we propose that comparing the sequence of ATCC 43816, NTUH-K2044, and MGH 78578 strains will reveal 
other factors that might play a role in the ability of the bacteria to cause the respiratory disease.

Due to the increase of MDR K. pneumoniae strains, there is an urgent need for the development of new antimicrobial agents, and to create the capability of noninvasive monitoring of therapeutic efficacy of clinically important drugs against $K$. pneumoniae lung infection, we decided to engineer a bioluminescent bioreporter strain of the bacteria. We propose that such strain will enable real-time monitoring of direct effect of antimicrobial agents on the organisms themselves instead of inferring the therapeutic efficacy from host physiology according to the current practice.

Lastly, K. pneumoniae causes an acute pneumonia in human but the bacteria have only few identified virulence factors as discussed above. In contrast, other pathogens capable of causing similar disease to the one caused by $K$. pneumoniae encode for a wide range of virulence determinants. Thus, this suggest that there are possibly more virulence determinants that might contribute in the ability of $K$. pneumoniae to cause the pulmonary disease, and the identification of these factors will reveal new vaccine and/or therapeutic targets. We propose that the identification of virulence determinants required by $K$. pneumoniae to cause the respiratory disease can be achieved using transposon sequencing (Tn-seq). 


\section{CHAPTER 2}

\section{MATERIALS AND METHODS}

\subsection{Bacterial strains and media}

K. pneumoniae strains were cultured routinely in Lennox Broth (LB) or LB agar plates at $37^{\circ} \mathrm{C}$. K. pneumoniae was preconditioned for cell culture and animal studies by subculturing overnight broth cultures 1:25 into TSBDC [178] for an additional $3 \mathrm{hr}$ of growth at $37^{\circ} \mathrm{C}$. Briefly, TSBDC is formulated as a concentrate of a $30 \mathrm{~g} / \mathrm{L}$ trypticase soy broth mixed with $5 \mathrm{~g} / \mathrm{L}$ of Chelex 100 in a $1 / 10^{\text {th }}$ volume, which is dialyzed from a $6-8 \mathrm{kDa}$ dialysis tubing into a $1 \mathrm{x}$ volume of $1 \%$ glycerol, where the media consists of the small organic compounds which leave the dialysis tubing into the $1 \%$ glycerol solution. TSBDC is supplemented with $50 \mathrm{mM}$ monosodium glutamate immediately prior to use. The bacterial cultures were washed into PBS and their concentration was estimated using $\mathrm{OD}_{600}$ measurements. The K. pneumoniae strains used in this study included ATCC 43816, MGH 78578 (kindly provided by Virginia Miller, UNC), NTUH-K2044 (kindly provided by Jin-Town Wang, NTUCM and Valley Stewart, UC Davis), and CIP 52.145 (Collection of Institut Pasteur). Where appropriate, antibiotics were used at the following concentrations unless otherwise stated: carbenicillin (100 $\mu \mathrm{g} / \mathrm{ml})$, kanamycin $(25 \mu \mathrm{g} / \mathrm{ml})$, zeocin $(100 \mu \mathrm{g} / \mathrm{ml})$, and gentamicin $(20 \mu \mathrm{g} / \mathrm{ml})$. 


\subsection{Sequencing of K. pneumoniae ATCC 43816}

ATCC 43816 genomic DNA was isolated from $\sim 5 \times 10^{9}$ bacteria grown in LB broth overnight. The DNA was isolated in TE buffer with $0.5 \%$ SDS extraction in the presence of proteinase $\mathrm{K}$ and $\mathrm{RNase}$, followed by phenol:chloroform:isoamyl alcohol $(25: 24: 1 \mathrm{v} / \mathrm{v})$ purification, and alcohol precipitation. A $1.5 \mu \mathrm{g}$ aliquot of chromosomal DNA was processed for Illumina Next Generation Sequencing based on the manufacturer's instructions. Briefly, fragmentase (NEB) was used to generate 100-300 bp DNA fragments which were end repaired, A-tailed, adaptor ligated, and PCR amplified using Phusion. Two lanes of 51 base reads were run to generate $22,422,915$ reads of sequencing data, filtered to eliminate low quality reads, and assembled using Velvet [179]. We assembled 1763 contigs, of which 1550 contigs were $>200$ bp and were deposited at DDBJ/EMBL/GenBank under the accession APWN00000000. The version described in this paper is the first version, APWN01000000. The contigs were aligned against the non-redundant nucleotide database using BLASTN [180] and hits to the full genomes of the NTUH-K2044 and MGH 78578 strains were retained separately. Manual sorting was conducted to identify contigs common to or unique from the NTUH-K2044 and MGH 78578 genomes, and unique sequence was aligned by BLASTN to identify homology to other bacterial species.

The capsular polysaccharide genetic cluster was manually sequenced to close contig gaps between five contigs, as described elsewhere [181], and the complete sequence for the ATCC 43816 capsular polysaccharide locus have 
been deposited with DDBJ/EMBL/GenBank with the accession number KJ541664.

\subsection{Quantification of capsular polysaccharide production}

Capsule production was quantified for K. pneumoniae from LB overnight cultures, as described elsewhere [182]. Briefly, PBS-washed bacteria were enumerated and subjected to hot phenol extraction before precipitating the chloroform-treated aqueous phase with $0.5 \mathrm{M}$ sodium acetate and then 10 volumes of $95 \%$ ethanol. Polysaccharide was pelleted at $7200 \mathrm{~g}$ for $5 \mathrm{~min}$ after an overnight storage at $20^{\circ} \mathrm{C}$. The pellet was resuspended in water, and uronic acid was measured from capsular polysaccharide preparations using a modified carbazole assay [183], with measurement calculated relative to a glucuronolactone standard.

\subsection{Generation of capsular polysaccharide mutants}

Capsular polysaccharide mutants were generated for $K$. pneumoniae strains ATCC 43816 and NTUH-K2044 by allelic exchange mutagenesis by initially PCR-amplifying upstream (5') and downstream (3') 1 kb fragments from a gene targeted for knock out (Table 1). The $1 \mathrm{~kb}$ homologous fragments were assembled in pSK (Stratagene) using a HindlII restriction site common to both the upstream and downstream fragments. A HindIII floxed zeocin cassette was inserted between the upstream and downstream fragments before the assembled construct was moved into an allelic exchange vector, pJMW106, which is a $\mathrm{Km}^{\mathrm{R}}$ variant of pCVD442 [184]. Thus, an Xbal-Kpnl fragment 
containing an in-frame $89.3 \%$ coding region deletion of the NTUH-K2044 wZC gene was cloned into pJMW106, and electroporated into E. coli strain S17-1 [185] to yield strain S17-1/pJMW106-NTUH $\Delta$ wzc::flox-zeo. Similarly, a XholSpel fragment containing an in-frame $90.4 \%$ coding region deletion of the ATCC 43816 manC gene was used to generate the strain S17-1/pJMW106-ATCC $\Delta \operatorname{manC::flox-zeo.}$

Allelic exchange was conducted over two stages, first by bacterial conjugation of the allelic exchange vectors from S17-1 to K. pneumoniae and selection of $\mathrm{Cb}^{R} \mathrm{Km}^{\mathrm{R}}$ merodiploid intermediates, and secondly by counter-selection of the suicide vector with $5 \%$ sucrose and zeocin. Confirmation of genome knock-out mutagenesis was confirmed on $\mathrm{Km}^{\mathrm{S}}$ clones using PCR analysis with 'mut' primers (Table 1) which flank the deletion site. The resulting strains were named ATCC $\triangle \operatorname{man} C$ and NTUH $\triangle W Z C$.

\subsection{Microscopic analysis of capsule mutants}

Negative staining of $K$. pneumoniae capsule was conducted using nigrosin stain, as described elsewhere [186]. Briefly, LB overnight broth cultures of wild type and capsule mutant strains of ATCC 43816 and NTUH-K2044 were mixed 1:1 with $10 \%$ nigrosin and smeared onto $18 \times 18 \mathrm{~mm}$ coverslips. The smear was air dried before mounting onto a glass slide. Samples were visualized with a $63 x$ objective on a Zeiss Axio microscope, and images were analyzed with Zeiss Axiovision Vs40x64 and Imaris x64 (Bitplane). 


\subsection{Macrophage uptake assay}

Both J774A.1 and RAW264.7 cell lines (ATCC) were cultured in DMEM (Invitrogen) supplemented with heat-inactivated 10\% fetal bovine serum (FBS, HyClone) and seeded into 96 well microtiter plates at a density of $7.5 \times 10^{4}$ or $2 \times 10^{5}$ cells per well, respectively. Cells were challenged at an $\mathrm{MOI}$ of 10 with $\mathrm{K}$. pneumoniae ATCC 43816, NTUH-K2044 or MGH 78578, or with capsule mutants ATCC $\triangle m a n C$ or NTUH $\Delta w z c$. At one hour post infection, gentamicin was added to eliminate extracellular bacteria $(20 \mu \mathrm{g} / \mathrm{ml}$ final, or $1000 \mu \mathrm{g} / \mathrm{ml}$ for the $\mathrm{Gm}^{\mathrm{R}} \mathrm{MGH} 78578$ strain). Gentamicin concentrations were empirically determined to kill extracellular K. pneumoniae in DMEM/FBS at $>99.99 \%$ efficiency within a $1 \mathrm{hr}$ window. At three hours post infection, monolayers were washed with PBS, lysed with $0.1 \%$ Triton X-100/PBS for 5 min, and serially diluted for bacterial enumeration on LB plates

\subsection{Macrophage survival assay}

J774A.1 and RAW264.7 macrophages were cultured in 96 well microtiter plates as described above. Triplicate wells of macrophages were infected with $K$. pneumoniae in five replicate plates, and infections were conducted for $1 \mathrm{hr}$ before the addition of gentamicin to kill extracellular bacteria, and antibiotic was maintained throughout the assay duration. At time points corresponding to 3 , $4.5,6,9$, and $12 \mathrm{hr}$ post infection, a microtiter plate of samples was washed in PBS before releasing intracellular bacteria from macrophages using a $5 \mathrm{~min}$ 
treatment of $0.1 \%$ Triton X-100/PBS. Samples were serially diluted in PBS and plated onto LB plates to enumerate intracellular bacteria.

\subsection{Intratracheal infection of mice}

Murine infection studies were approved by the University of Louisville Institutional Animal Care and Use Committee in accordance with National Institutes of Health guidelines (Protocol \# 10069). Groups of five 8 wk old female BALB/c mice (Charles River) were challenged using a non-surgical intratracheal infection procedure was developed to minimize trauma during pathogen delivery. Intubation-mediated intratracheal (IMIT) inoculations were conducted as demonstrated in detail elsewhere [187]. Briefly, isoflurane-anesthetized animals received $10 \mu \mathrm{l}$ of $2 \%$ lidocaine anesthetic to the rear of the throat and were supported supine on a tilting platform raised to a $45^{\circ}$ angle. Using a fine tipped cotton applicator, the tongue was retracted while an otoscope fitted with a cutaway specula was inserted into the oral cavity to visualize the glottis. An $18 \mathrm{G}$ catheter, cast with a silicone rubber sleeve (10 $\mathrm{mm}$ of catheter exposed) was used to intubate mice, using a 20 mil guide wire to assist catheter placement. A 20 G blunt needle was used to instill a $50 \mu \mathrm{I}$ PBS bacterial suspension directly into the lung via the catheter, followed by a $150 \mu \mathrm{l}$ volume of air to aid distribution of the inoculum. Infected animals were monitored twice daily for indications of moribund disease, at which point they were humanely euthanized by isoflurane. Studies were concluded at 14 days. 


\subsection{Bacterial enumeration from key sites of infection}

Groups of five BALB/c mice were infected using the IMIT model with $10^{2.2} \mathrm{CFU}$ of either NTUH-K2044 or ATCC 43816. Moribund mice were euthanized at the presentation of lethargy, hunching, and labored breathing. Mice were euthanized by overdose of isoflurane, immediately followed by exsanguination by cardiac puncture with a $23 \mathrm{G}$ needle, and the blood was collected to a Microtainer ( $\mathrm{K}_{2}$ EDTA, BD Biosciences). Lung, liver and spleen were each collected into a sterile Whirl-Pak bag (Nasco) and homogenized in $1 \mathrm{ml}$ of sterile PBS, by rolling the tissue with a $25 \mathrm{ml}$ serological pipette. Blood and tissue homogenate were subjected to detergent lysis with $1 \%$ Triton $\mathrm{X}-100$ for 5 min and subsequently serially diluted in a 96 well plate. LB plates were spot-plated with $10 \mu \mathrm{l}$ aliquots of diluted bacterial suspensions, grown for $8 \mathrm{hr}$ at $37^{\circ} \mathrm{C}$, and bacterial burdens were calculated based on dilution factor, tissue weight, and estimated tissue density. Neutral buoyancy testing in glycerol solutions revealed that the estimated tissue densities for lung, liver, and spleen were 1.03, 1.08, and 1.06 $\mathrm{g} / \mathrm{ml}$, respectively.

\subsection{Creating the bioluminescent JSKP001 strain}

The bioluminescent JSKP001 strain was created by the insertion of a lux operon in-between fus $A$ and yee $F$ genes using two-stage allelic exchange mutagenesis as described previously [99]. Briefly, 1 kb upstream (5') and downstream (3') fragments flanking the insertion site was PCR amplified from ATCC 43816 genome using primers listed in table 1 and cloned into pSK. A DNA fragment of 
407-bp containing a stem loop terminator and $r p / U$ promoter was also generated from K. pneumoniae genome and combined with the 5' fragment using BsiWI cut site engineered in both fragments. Both 5' and 3' handles were assembled in pGSVS suicide vector on either ends of the lux operon generating the pGSVSKpin-Prp/U-Iux plasmid. A loxP-flanked (floxed) zeocin antibiotic marker (Sh ble) was PCR amplified and cloned into pSK creating pSK-floxZeo. The floxZeo fragment was subcloned between the 3 ' handle and lux $C$ open reading frame of the lux operon in the plasmid pGSVS-Kpin-Prp/U-lux creating the construct pGSVS2-Kp ATCC-PrpIU-lux-floxZeo. The constructed pGSVS2-Kp ATCCPrp/U-lux-floxZeo vector was electroporated into S17-1, conjugated into $K$. pneumoniae ATCC 43816, and using two-stage allelic replacement, the ATCC 43816::Kp ATCC-Prp/U-lux-floxZeo strain was created and named WKP001. The zeocin marker was removed from the WKP001 strain using cre recombinase system. Briefly, a 1-Kb fragment containing galF gene was PCR amplified, cloned into pSK giving the plasmid pSK-galF, and then the same fragment was subcloned into pKSVS-Pto/C-cre using Spel/Notl restriction sites creating the vector pKSVS-Pto/C-cre-galF. S17-1::pKSVS-Pto/C-cre-galF was conjugated into WKP001 and with the trans expression of cre, the zeocin cassette was removed generating the JSK001 strain.

\subsection{In vitro proliferation of JSKP001 and ATCC 43816}

Overnight cultures of the JSKP001 and ATCC 43816 strains were diluted 1000 fold in LB broth and $100 \mu \mathrm{l}$ aliquots (in triplicate for each strain) were transferred 
into Greiner 96-well black plate with clear bottom. A kinetic read was performed in Synergy ${ }^{\mathrm{TM}} \mathrm{H} 1$ monochromator-based multi-mode microplate reader (BioTek) where the machine was setup to take $\mathrm{OD}_{600}$ and bioluminescence measurements at 10 minute intervals for 4 hours at $37^{\circ} \mathrm{C}$ with agitation.

\subsection{In vitro correlation of JSKP001 bioluminescence and bacterial numbers}

Overnight culture of JSKP001 strain was diluted 1000 fold into $4 \mathrm{ml} \mathrm{LB}$ broth media and grown for $3 \mathrm{hr}$ at $37^{\circ} \mathrm{C}$ with agitation in an orbital shaker (200 rpm). $100 \mu \mathrm{l}$ aliquots (in triplicate) were taken at 30 minute intervals for bioluminescence estimation using Synergy ${ }^{\mathrm{TM}}$ HT Multi-Detection Microplate Reader (BioTek) and for bacterial numbers enumeration.

\subsection{In vivo fitness and real-time monitoring of $K$. pneumoniae in mice}

All animal studies were approved by the University of Louisville Institutional Animal Care and Use Committee (IACUC no. 10069 and 13059). Two groups of five female albino C57BL/6J mice 8-10 weeks of age (Jackson Laboratories) was infected intratracheally with ATCC $43816\left(10^{4.95} \mathrm{CFU}\right)$, and JSKP001 (10 4.91 $\mathrm{CFU}$ ) as described elsewhere [187]. Bioluminescence imaging (BLI) was conducted using PhotonIMAGER Optima system (BIOSPACE LAB) and health checks were performed twice daily. The animals were humanely euthanized upon meeting heart rate of 400 beat per minute (BPM) or below, monitored using MouseOx Plus (STARR Life Sciences Corp.), in addition to illustration of clear 
signs of disease including slow to no movement, minimum resistance upon handling, hunching, and shortness of breath (SOB). The euthanasia was conducted with isoflurane followed by exsanguination using cardiac puncture. Upon reaching moribund disease, blood, BAL, lung, liver, spleen, and kidneys were collected and processed for bacterial enumeration as described previously [99]. In vivo monitoring of bacterial colonization of host lungs for each animal was

performed by $\mathrm{BLI}$, where region of interests (ROls, $5 \mathrm{~cm}^{2}$ ) were drawn on mice thoracic cavity (imaged for 1 minute on dorsal position) with technical background subtraction. Two standard deviation limit of detection (LOD) was established from uninfected mice (biological background).

\subsection{Measuring the correlation between bioluminescence and bacterial numbers within host tissues}

Female albino C57BL/6J mice were infected with $10^{3}-10^{5.2}$ CFU with JSKP001 strain and lung, liver, spleen, kidneys, $100 \mu \mathrm{BAL}$, and $100 \mu \mathrm{l}$ blood samples were collected from mice that developed moribund disease $(n=36)$. Bacterial burdens were estimated by measuring bioluminescence in Greiner 24-well black plates using PhotonIMAGER Optima system followed by processing of samples for bacterial enumeration as described previously [99]. Correlation between in vivo bioluminescence in life and bacterial burdens were performed by drawing 5 $\mathrm{cm}^{2}$ ROls on animals' thoracic cavity right before euthanasia to estimate bioluminescence levels and bacterial loads were enumerated from lung 
homogenates following euthanizing the moribund mice $(n=29)$ and processing the tissues as described above.

\subsection{MicroChem-Plus killing assay}

Eighteen hours overnight bacterial culture was diluted 25 fold in LB and the microbes were grown to exponential phase for $1 \mathrm{hr}$ at $37^{\circ} \mathrm{C}$ with agitation. The bacteria were centrifuged at $15000 \mathrm{~g}$ for 1 minute, the supernatant was decanted, the pellets were resuspended in sterile PBS, and mixed in 1:1 ratio with serially diluted 2x MicroChem-Plus disinfectant in Greiner 96-well black plate with clear flat bottom. 10 min kinetic read was immediately performed in Syngery ${ }^{\top \mathrm{M}} \mathrm{H} 1$ plate reader at room temperature $\left(\sim 24^{\circ} \mathrm{C}\right)$ where the machine was setup to measure bioluminescence every minute and following 11 min of incubation, the bacteria were rapidly diluted and spot plated on LB agar plates for enumeration.

\subsection{In vitro bacterial growth inhibition by meropenem}

Overnight bacterial suspension was diluted 25 fold in LB broth and the microbes were grown further to exponential phase at $37^{\circ} \mathrm{C}$ for 1 hour with agitation. The exponentially growing bacteria $\left(10^{8.9} \mathrm{CFU} / \mathrm{ml}\right)$ were diluted 50 fold in $\mathrm{LB}$ and mixed in 1:1 ratio with $2 x$ serially diluted meropenem in 96 -well black Greiner plate with clear bottom. A kinetic read in Synergy ${ }^{\top \mathrm{M}} \mathrm{H} 1$ plate reader was performed at $37^{\circ} \mathrm{C}$ where $\mathrm{OD}_{600}$ and bioluminescence measurements were taken every $5 \mathrm{~min}$ for $8 \mathrm{hr}$. The $50 \%$ effective dose $\left(E_{50}\right)$ of the drug was estimated at the $8 \mathrm{hr}$ time point using GraphPad Prism 6 software. 


\subsection{Meropenem therapeutic study}

Four groups of female albino C57BL/6J mice (8-10 weeks of age) were infected intratracheally with a lethal dose $\left(10^{4.9} \mathrm{CFU}\right)$ of $K$. pneumoniae JSKP001 strain and three hours post infection, three groups was subcutaneously introduced with either 96, 200, or $400 \mathrm{mg} / \mathrm{kg} /$ day meropenem prepared in PBS (HyClone) while the fourth group was injected with vehicle only (PBS). Injecting the therapeutic treatments, monitoring animals' health status (including heart rate measurement), and optical diagnostic imaging was performed $\mathrm{q} 12 \mathrm{~h}$. The animals were monitored for one week post infection and humanely euthanized upon meeting the endpoint criteria explained earlier. The $96 \mathrm{mg} / \mathrm{kg} / \mathrm{day}$ group was conducted in a separate study from the 200 , and $400 \mathrm{mg} / \mathrm{kg} /$ day treatment groups.

\subsection{Creating the bioluminescent man $C$ and vgrG mutants}

The bioluminescent $\operatorname{man} C$ and $v g r G$ mutants were created using allelic exchange mutagenesis as described previously [99]. The JSKP001 $\Delta$ manC strain was created from the previously made strain ATCC $43816 \Delta$ manC [99], where a lux operon was inserted into this strain using the plasmid pGSVS2-Kp ATCC-Prp/Ulux-floxZeo transformed into ATCC through conjugation from S17-1 followed by two step allelic replacement generating the strain WKP001 $\Delta$ manC. The insertion of the lux operon into bacterial genome was confirmed by testing bioluminescence production using Synergy ${ }^{\mathrm{TM}} \mathrm{HT}$ plate reader and/or PhotonIMAGER Optima system. The zeocin marker was removed from the 
WKP001 $\triangle$ manC using cre recombinase system as described above creating the strain JSKP001 $\Delta$ manC. On the other hand, the bioluminescent $\operatorname{vgrG}$ mutants were made in the JSKP001 strain. $1 \mathrm{~kb}$ upstream (5') and downstream (3') fragments from vgrG1, and vgrG2 genes were PCR amplified and combined in pSK (table 1). Zeocin cassette was inserted in-between the handles using HindIII cut site, shared between the two fragments, creating the plasmids pSK-vgrG1flox-zeo and pSK-vgrG2-flox-zeo followed by subcloning the assembled vgrGflox-zeo fragments into pJMW106. The resulted constructs were transformed into S17-1 E. coli competent cells creating the strains S17-1/pJMW106-vgrG1-floxzeo and S17-1/pJMW106-vgrG2-flox-zeo. These vectors were transformed into JSKP001 by conjugation and following two step allelic replacement the

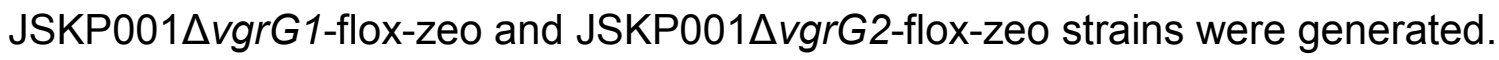
The zeocin markers were removed from those strains using the cre recombinase system described previously creating JSKP001 $\Delta$ vgrG1 and JSKP001 $\Delta$ vgrG2 strains. The bioluminescent vgrG1/2 double knockout was generated in the JSKP001 $\Delta v g r G 2$ strain using the same steps. The deletion of the $v g r G$ genes were confirmed with PCR using vgrG 'mut' primers (table 1).

\subsection{Testing the fitness of the JSKP001 $\Delta$ manC in vivo}

Female albino C57BL/6J mice (8-10 weeks of age) were infected with either JSKP001 (104.1 CFU) and JSKP001 $\Delta$ manC (10 $\left.{ }^{8.0} \mathrm{CFU}\right)$ using IMIT and monitored for two weeks for the development of moribund disease which consist of a heart rate of $400 \mathrm{bpm}$ or below in addition to illustration of severe illness 
including slow movement, reduced resistance upon handling, hunching, and SOB. Moreover, bacterial proliferation within mice lungs were monitored using PhotonIMAGER Optima system every $12 \mathrm{hr}$. Animals were euthanized upon meeting moribund disease or two weeks post infection and blood, BAL, lungs, liver, spleen, and kidneys were collected for bacterial burden estimation.

\subsection{Monitoring JSKP001 $\Delta$ manC early clearance in vivo}

Eight to ten weeks of age female albino C57BL/6J mice were infected with $10^{7.9}$ CFU of the JSKP001 1 manC strain using IMIT. Bioluminescence imaging (BLI) monitoring bacterial lung colonization was initiated right after the infection (0.5 hour post infection) followed by imaging the mice at $3 \mathrm{hr}$ intervals. The animals were euthanized 6.5 hours post infection, lungs were collected, and processed for bacterial burden estimation as previously described.

\subsection{Tracking JSKP001 replication within cultured macrophages}

Time course gentamicin protection assay was performed as described above. Briefly, RAW264.7 murine macrophages were infected with $\mathrm{MOI}$ of 10 with the JSKP001 and ATCC 43816 (in triplicate) using four replicate Greiner 96-well flat bottom black plates. Gentamicin treatment was performed $1 \mathrm{hr}$ post infection to kill all extracellular bacteria. Bacterial loads within the cells were estimated at 3 , 4.5, 6, and 9 hr time points by measuring bioluminescence using Synergy ${ }^{\top \mathrm{M}} \mathrm{HT}$ plate reader followed by lysing the cells with $0.1 \%$ Triton X-100 (in PBS) for 5 min for bacterial enumeration. 


\subsection{Creating bioluminescent $K$. pneumoniae strains with different}

\section{promoters}

pSK-Kp5'-Pto/C plasmid was reverse PCR amplified adding the sequence for the synthetic EM7 promoter and ATCC 43816 frr, ompC, and rpoD promoters together with Nhel restriction sites (table 1), which then got ligated and transformed into DH10B creating the strains DH10B/pSK-Kp5'-PEM7,

DH10B/pSK-Kp5'-PrpoD, DH10B/pSK-Kp5'-Pfrr, and DH10B/pSK-Kp5'-PompC. The Kp5' handle with the different promoters were subcloned into pGSVS using EcoRI/PspOMI cut sites, and then larger fragments including the Kp5' handles together with the different promoters and part of the lux operon were subcloned into pJMW106-Kp-nif-PtolC-lux-floxZeo replacing the Pto/C promoter with either EM7, frr, rpoD, or ompC promoters creating the vectors pJMW106-Kp-nif-PEM7lux-floxZeo, pJMW106-Kp-nif-Pfrr-lux-floxZeo, pJMW106-Kp-nif-PrpoD-luxfloxZeo, and pJMW106-Kp-nif-PompC-lux-floxZeo. Those constructs were transferred from S17-1 into ATCC 43816 by conjugation and a two-step allelic replacement took place as described above creating the strains ATCC::Kp ATCC-PEM7-Iux-floxZeo, ATCC::Kp ATCC-Pfrr-lux-floxZeo, ATCC::Kp ATCCPrpoD-lux-floxZeo, and ATCC::Kp ATCC-PompC-Iux-floxZeo. Cloning of the different promoters into the aforementioned plasmids were confirmed by sequencing, and the creation of the different bioluminescent strains were validated by testing light emission using Synergy ${ }^{\mathrm{TM}} \mathrm{HT}$ plate reader and/or PhotonIMAGER Optima. 


\subsection{Monitoring the bioluminescent $K$. pneumoniae strains replication within macrophages}

J774A.1 cells (seeded in Greiner 96-well black plate with flat bottom) were infected with MOI of 10 with the strains ATCC::Kp ATCC-PEM7-lux-floxZeo, ATCC::Kp ATCC-Pfrr-lux-floxZeo, ATCC::Kp ATCC-PrpoD-lux-floxZeo, and ATCC::Kp ATCC-PompC-lux-floxZeo and the plate was incubated at $37^{\circ} \mathrm{C}$. Extracellular bacteria were killed $1 \mathrm{hr}$ post infection using gentamicin treatment as described above. Intracellular bacteria were monitored by measuring bioluminescence at $2,3,4,5,6,9$, and $12 \mathrm{hr}$ using Synergy ${ }^{\mathrm{TM}} \mathrm{HT}$ plate reader.

\subsection{Estimation of intracellular bacteria within host lungs}

Albino C57BL/6J mice were infected with JSKP001 (105.1 CFU) using IMIT and their health status was monitored every $12 \mathrm{hr}$. Animals were euthanized $43 \mathrm{hr}$ post infection and lungs were collected for total and intracellular bacterial enumeration. The tissues were dissociated into single cell suspension using $0.3 \%$ collagenase treatment (in PBS) for $2.5 \mathrm{hr}$ at room temperature followed by running the homogenates through cell strainer to separate the tissue clumps into single cells. A portion of the cell suspension was treated with $1 \%$ Triton X-100 to release intracellular microbes for total bacterial estimation. Intracellular bacteria were estimated using gentamicin protection assay and a newly developed filtration technique. The amount of viable cells in the suspension was estimated using hemocytometer following trypan blue staining. Gentamicin was used on $10^{6}$ cells suspended in $1 \mathrm{ml}$ PBS to eliminate extracellular bacteria using $1 \mathrm{mg} / \mathrm{ml}$ 
treatment which we determined that it would kill $99.98 \%$ of the bacteria at room temperature following the $1 \mathrm{hr}$ treatment (Figure 1). The cells were spun down at $100 \mathrm{~g}$ for $5 \mathrm{~min}$ and a couple of PBS washes were preformed to get rid of the antibiotic. The cell pellet were resuspended in PBS, and lysed for bacterial enumeration. For estimating the intracellular bacteria using the filter technique, $10^{6}$ cells in $1 \mathrm{ml}$ PBS were passed through 5 micron filter assembled in swinnex filter holder and two PBS washes were performed to get rid of the extracellular bacteria. To release the intracellular microbes trapped in the membrane, $1 \%$ Triton X-100 (in PBS) treatment was performed, the eluted bacteria was collected, and enumerated. Following preforming the gentamicin killing and the filter techniques, the percentages of intracellular bacteria to the total microbial numbers were estimated. 


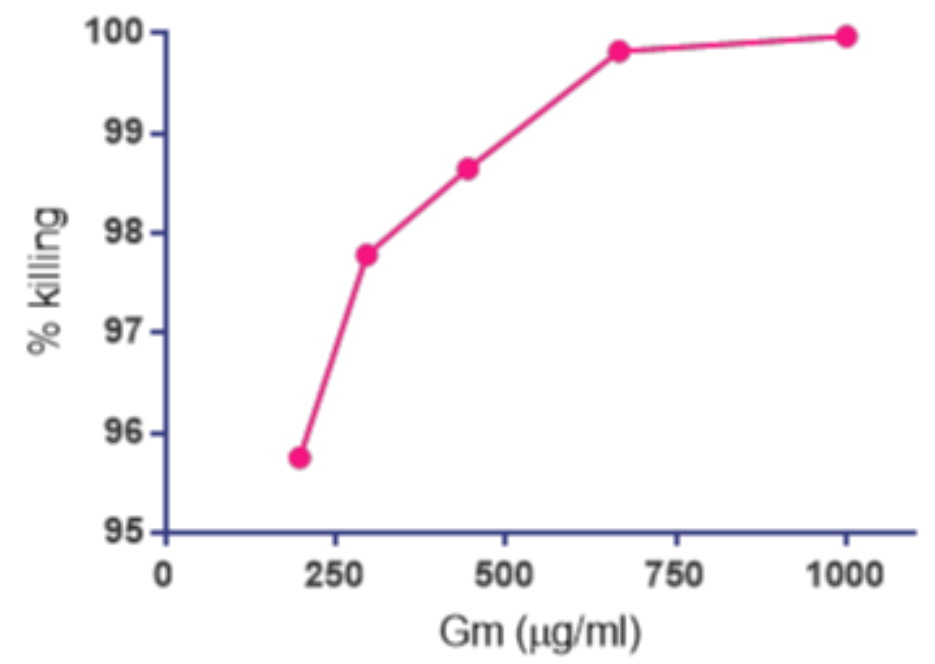

Figure 1. Gentamicin killing of $K$. pneumoniae at room temperature.

$2 \times 10^{6} \mathrm{CFU} / \mathrm{ml}$ bacterial suspension in PBS was prepared using overnight culture (18 hr) of ATCC 43816 and treated with a serially diluted gentamicin (from 17.34 to $1000 \mu \mathrm{g} / \mathrm{ml}$ ). The bacterial suspensions were incubated with the different antibiotic concentrations for $1 \mathrm{~h}$ at room temperature $\left(22^{\circ} \mathrm{C}\right)$ and following the $1 \mathrm{~h}$ treatment, the bacteria were centrifuged at $15000 \mathrm{~g}$, and two PBS washes were performed to get rid of the antibiotic. The bacteria were spot plated on LB plates for bacterial enumeration. 


\subsection{Tn-seq study}

\subsubsection{Creating transposon library in JSKP001}

pSAM-RF-Km plasmid was created from the vector pSAM-DKm (kindly provided by Dr. Yoder-himes) by deleting the bla gene to eliminate the carbenicillin resistance. pSAM-DKm was digested using Pvul/ApaLI restriction enzymes, the ends were blunted using DNA Polymerase I, Large (Klenow) Fragment, ligated for overnight using T4 DNA ligase, and transformed into E. coli S17-1 creating the strain S17-1/pSAM-RF-Km. pSAM-RF-Km plasmid was transferred into JSKP001 using conjugation (4:1 ratio) at the $37^{\circ} \mathrm{C}$ for 1 hour followed by spreading $100 \mu \mathrm{l}$ aliquots of the conjugation mixture on 42 LB plates with $\mathrm{Cb} 100 \mathrm{Km} 25$ selection and growing the microbes at $37^{\circ} \mathrm{C}$ for 14 hours. A total of 20000 (or $10^{4.2} \mathrm{CFU}$ ) transposon mutants were collected (4x genome coverage of JSKP001), grown in LB broth for overnight, and stored in the $-80^{\circ} \mathrm{C}$ in $25 \%$ glycerol.

\subsubsection{Infecting albino C57BL/6J mice with the generated Tn-seq library} Female albino C57BL/6J mice (8-10 weeks) were infected with $10^{4.8} \mathrm{CFU}$ of the JSKP001 transposon mutants using IMIT. Bacterial lung colonization was monitored using PhotonIMAGER Optima system every $12 \mathrm{hr}$ and then we switched into $3 \mathrm{hr}$ monitoring upon meeting in vivo lung bioluminescence $\geq 10^{6.5}$ $\mathrm{ph} / \mathrm{s}$. Mice were euthanized after reaching or exceeding in vivo lung bioluminescence of $10^{8} \mathrm{ph} / \mathrm{s}$. Lungs were collected and processed as described above for bacterial enumeration. Bacterial inoculum used to infect the animals 
(input library) and bacteria extracted from tissue homogenates (output libraries) were inoculated into LB broth and cultured for overnight at $37^{\circ} \mathrm{C}$ with agitation (200 rpm) to expand the bacterial libraries. On the following day, the cultures were centrifuged and the bacterial pellets were frozen and stored at $-80^{\circ} \mathrm{C}$ to be used for genomic DNA extraction and the subsequent steps for Tn-seq analysis including Mmel digest, agarose gel electrophoresis, DNA purification, adaptor ligation, PCR amplification, and massive parallel sequencing (MPS).

\subsection{Testing the virulence of the vgrG mutants in single strain infection model}

Female albino C57BL/6J mice were infected with $10^{4.8}-10^{4.9} \mathrm{CFU}$ of the

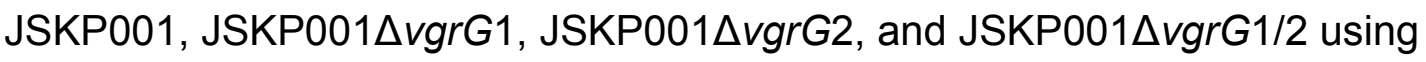
IMIT. Animals were monitored for the development of moribund disease and euthanized upon meeting heart rate of $400 \mathrm{bpm}$ or below in addition to the illustration of clear signs of illness including reduced mobility, minimum resistance upon handling, SOB, and hunching. Upon euthanasia, blood, BAL, lung, liver, spleen, and kidneys were collected for bacterial burden estimation as described above.

\subsection{Testing vgrG mutants fitness using in vivo competition study} ATCC 43816 was inoculated into female albino C57BL/6J mice in 1:1 ratio with

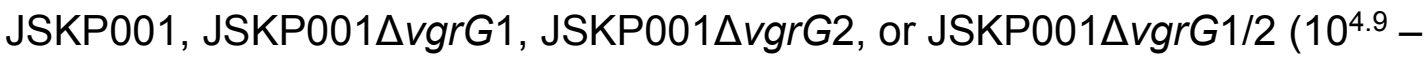
$\left.10^{5.4} \mathrm{CFU}\right)$. In vivo bacterial colonization of mice lungs were monitored using 
PhotonIMAGER Optima system every $12 \mathrm{hr}$, animals were euthanized upon meeting heart rate of $\leq 500$, and blood, lung, liver and spleen tissues were collected for bacterial burden estimation. The JSKP001 strains were distinguished from the non-luminescent ATCC 43816 bacteria by taking a picture of the LB plates using PhotonIMAGER Optima system. The competitive indexes were calculated by dividing ratios of the JSKP001 strains to ATCC 43816 in the tissues by the ratios of the JSKP001 strains to ATCC 43816 in the inoculums.

\subsection{Statistical analysis}

Statistical analysis of data sets was conducted by One-way ANOVA with Tukey post-test of log-transformed data, survival data was conducted using Log-rank (Mantel-Cox) and Gehan-Breslow-Wilcoxon Tests (GraphPad Prism 5). Probit analysis (StatPlus 2009 Professional) was used to calculate the $\mathrm{LD}_{50}(50 \%$ Lethal Dose) and both the upper (UCL) and lower (LCL) reliable interval values in addition to meropenem $\mathrm{ED}_{50}$ (50\% effective dose). In vitro growth curves were analyzed using unpaired student T-test, using log transformed OD600 reads. Bacterial burden in tissues of animals infected with either JSKP001 or ATCC 43816 strains were compared by Two-way ANOVA with Bonferroni post-test of log transformed data. Unpaired student T-test was used to analyze the difference in meropenem $\mathrm{EC}_{50}$ ( $50 \%$ effective concentration) values estimated by $\mathrm{OD}_{600}$ and bioluminescence against JSKP001 (GraphPad Prism 6). 
Table 1. Primers used in this study.

\begin{tabular}{|c|c|}
\hline 5' ATCC manc Xbal (+) & $\begin{array}{l}\text { CTGCTCGAGATTACCAAAGATATCTTCACCAAGAAGGATGA } \\
\text { AG }\end{array}$ \\
\hline ATCC manC HindIII (-) & GTAAAGCTTGCGAGACATCGGCCAGAGACGAC \\
\hline ATCC manC HindIII (+) & TGAGATCCAGTCGGGGTCGTACCTC \\
\hline ATCC manC Kpnl (-) & STTTTCGCTCCCGGCTGCTTCTGC \\
\hline ATCC manC mut (+) & $\begin{array}{l}\text { GTTATTCTACAATAAACTGACCAAGTCATCTTGTTTCCTCTC } \\
\text { CTTCG }\end{array}$ \\
\hline ATCC & CTATCTTCCCGGGTTTCAGAAATTCGCCGTAGGC \\
\hline 5' NTUH wzc Xbal (+) & $\begin{array}{l}\text { CGTTCTAGAGCATAACGGTAAAGATACTAAGATCTCCTTATA } \\
\text { TGC }\end{array}$ \\
\hline 5' NTUH wzc HindIII (-) & $\begin{array}{l}\text { CAAAGCTTTATGATCAATAACTTCACCAATTAAACGACCTAG } \\
\text { ATCGATCC }\end{array}$ \\
\hline 3' NTUH wzc HindllI (+) & $\begin{array}{l}\text { CAAAGCTTTCGATGTTGCTAAAAATAGATTGGAACATAGCG } \\
\text { GTGTTATAG }\end{array}$ \\
\hline H wzc Konl (-) & $\begin{array}{l}\text { CTGGTACCTAATAATGAGGAGAACATTACCATAAAACGAGA } \\
\text { TGTATTTCG }\end{array}$ \\
\hline & GGCAAAACTATGTTATTCGGACATTGGATAGGGCAACGAG \\
\hline NTUH wze mut (-) & $\begin{array}{l}\text { CATTAATCGCAAGGCCAAATCCTTGTGATAATAC } \\
\text { AGTATTC }\end{array}$ \\
\hline 5' ATCC vgrG1 Xbal (+) & GTCTAGAAACCGCGCTTA \\
\hline 5' ATCC vgrG1 HindIII (-) & CCAAGCTTCAC \\
\hline 3' ATCC vgrG1 Hindlll (+) & CAAAGCTTGTC \\
\hline 3' ATCC vgrG1 Kpnl (-) & CCGGTACCCATTTTGGAGGC \\
\hline ATCC vgrG1 mut (+) & CCGTAACGTCC \\
\hline G1 mut (-) & CTCAGGTAGCC \\
\hline rG2 Xbal (+) & CGTCTAGATGATCGTGGTGGCAGGCCATACC \\
\hline 5' ATCC vgrG2 HindIII (-) & GCAAGCTTGTAACGGTTAAGGGTGGTGCCGGTAATTATTG \\
\hline 3' ATCC vgrG2 Hindlll (+) & CGAAGCTTCGTAATACTTACGCGGATTTGCTCGATGAC \\
\hline 3' ATCC vgrG2 Kpnl (-) & GCGGTACCCCAGTACTCATCGAGCATATTGTGTC \\
\hline ATCC vgrG2 mut (+) & GGGTTACCTTTTAATTACGAAACTCCTGGTGGGGTATC \\
\hline ATCC vgrG2 mut (-) & CGATTTTTGCGCCTTTTCGATGGCTCGATATACCGC \\
\hline pSK-Kp5'-Ptc & CCGGCTAGCAAAAACGGGAGCCCATCGGCTC \\
\hline (EM7) & $\begin{array}{l}\text { CATGCTAGCGTTGACAATTAATCATCGGCATAGTATATCGG } \\
\text { CATAGTATAATACGACTCAGGGCCGAT GGGCCCGGTACCC } \\
\text { AATTCGCCCTATAG }\end{array}$ \\
\hline $\begin{array}{ll}0 \\
(+)\end{array}$ & $\begin{array}{l}\text { GAAGCTAGCGCCGGTGCTTTACAAAGCAGCAGTAGTTGCA } \\
\text { GTAAAATTCCGCACCATTTTGAAATGATGGGCCCGGTACCC } \\
\text { AATTCGCCCTATAG }\end{array}$ \\
\hline $\begin{array}{l}\text { pSK-Kp5'- PtolC (frr) } \\
\text { Nhel/PspOMI (+) }\end{array}$ & $\begin{array}{l}\text { CACGCTAGCTCAACAAGAGTTGGTGTCTGGATGGATTTTGT } \\
\text { GGTATAAAGCGCGCCGGACTTCCGGATGGGCCCGGTACC } \\
\text { CAATTCGCCCTATAG }\end{array}$ \\
\hline $\begin{array}{l}\text { pSK-Kp5'- PtolC ( } \\
\text { Nhel/PspOMI (+) }\end{array}$ & $\begin{array}{l}\text { GAAGCTAGCGTATCATATTCTTGTTGGATTATTCTGCATTTT } \\
\text { GCAGCACAATGAAATAGCCGACTGAGATGGGCCCGGTACC } \\
\text { CAATTCGCCCTATAG }\end{array}$ \\
\hline
\end{tabular}




\title{
CHAPTER 3
}

\section{CORRELATION OF KLEBSIELLA PNEUMONIAE COMPARATIVE GENETIC}

\author{
ANALYSES WITH VIRULENCE PROFILES IN A MURINE RESPIRATORY
}

\author{
DISEASE MODEL ${ }^{1}$
}

\subsection{Introduction}

Klebsiella pneumoniae ssp. pneumoniae (K. pneumoniae) is responsible for emerging infectious disease and is a causative agent of both nosocomial and community acquired pneumonia (CAP) worldwide. The epidemiology of $K$. pneumoniae is complex, involving ecological persistence as well as carriage in both animal and human populations [188]. Carriage of $K$. pneumoniae is frequently associated with colonization of the upper respiratory tract or gastrointestinal (GI) tract, with the potential for GI tract amplification of antibiotic resistant strains of $K$. pneumoniae following antibiotic therapies [189]. $K$. pneumoniae opportunistically infects a variety of mucosal surfaces with the primary sites of infection including the urinary tract and the lower respiratory tract (LRT) [190].

\footnotetext{
${ }^{1}$ Fodah, R. A., Scott, J. B., Tam, H. H., Yan, P., Pfeffer, T. L., Bundschuh, R., \& Warawa, J. M. (2014). Correlation of Klebsiella pneumoniae comparative genetic analyses with virulence profiles in a murine respiratory disease model. PLoS One, 9(9), e107394. doi:10.1371/journal.pone.0107394
} 
K. pneumoniae pneumonia is a fatal disease with mortality rates of up to $22.7 \%[48,191]$. The incidence of $K$. pneumoniae pneumonia in the United States is more commonly associated with nosocomial acquisition of disease rather than environmental sources [14, 192], as Klebsiella is thought to contribute to only $1 \%$ of CAP in North America [193-195]. K. pneumoniae is the fifth most prevalent nosocomial bacterial pathogen in the United States for infections associated with UTIs, VAP and central line-associated bacteremia, and accounts for $6 \%$ of all nosocomial bacterial disease [196]. The emergence of $K$.

pneumoniae as a nosocomial pathogen in the US and Europe may be due in part to the acquisition of antibiotic resistance markers providing a selective advantage in hospital settings, with particular concerns growing over an increasing prevalence of carbapenemase-expressing K. pneumoniae (KPC) strains [197]. Well characterized outbreaks of KPC dating back to 1988 have still not led to effective clinical diagnosis or control of these emerging pathogens in the US over 20 years later $[80,198]$. Recently instated surveillance programs have begun to characterize the increasing threat of $K$. pneumoniae in the US health care system, where it is understood that the threat of $K$. pneumoniae, and in particular KPC, may be underestimated, particularly in long-term acute care facilities [199]. Several virulence determinants are important in mediating the virulence of K. pneumoniae in the lung, including capsular polysaccharide, lipopolysaccharide, enterobacterial common antigen, OmpA, OmpK36, the AcrAB efflux pump, the regulator RamA, the biofilm related factor Ycil, and yersiniabactin [92, 156, 159, 200-204]. However, additional undescribed systems 
may participate in K. pneumoniae virulence. To this end, numerous sequencing efforts have begun to characterize the genomes of $K$. pneumoniae strains including the first whole genome sequences of the clinical isolates MGH 78578 and NTUH-K2044 [205], and the more recent release of whole genome sequences of the strains HS11286 [206] and KCTC 2242 [207]. Comparative genetic analyses have successfully led to the identification and characterization of a novel allantoin metabolism locus required for $\mathrm{Gl}$ tract disease which is present in the genome of NTUH-K2044, but absent from MGH 78578 [93].

The ATCC 43816 strain has been the focus of several studies characterizing the respiratory disease caused by $K$. pneumoniae [92, 200, 208], however this strain has not been sequenced, thus limiting investigations directed at identification of novel virulence determinants. To address this scientific gap, we have performed whole genome sequencing of ATCC 43816 to begin the characterization potential genomic differences relative to the sequenced $K$. pneumoniae strains.

We also decided to investigate host-pathogen interaction for the sequenced K. pneumoniae strains NTUH-K2044 and MGH 78578, for which little is known about their ability to cause respiratory disease or interact with professional phagocytes. We developed a novel pulmonary-specific delivery respiratory disease model in which to examine sequenced $K$. pneumoniae strains. Finally, we have characterized the ability of $K$. pneumoniae to modulate uptake and persist within multiple cultured murine macrophage cell lines. 


\subsection{Results}

\subsubsection{Whole genome sequencing of strain ATCC 43816}

K. pneumoniae ATCC 43816 is a well-studied strain, capable of causing a moribund respiratory disease in mouse models. However, limited genomic data is available to support future investigations of $K$. pneumoniae pathogenesis, thus we decided to perform whole genome sequencing. Next Generation Sequencing was used to sequence the ATCC 43816 genome, which was subsequently assembled into 1763 contigs consisting of $4.207 \mathrm{MB}$ of sequence data (accession number APWN00000000). To investigate the genetic relatedness of sequenced K. pneumoniae strains, the ATCC 43816 genome was aligned to the complete genome sequences of the NTUH-K2044 and MGH 78578 strains (Figure $2 \mathrm{~A}$ and $\mathrm{B}$, respectively). A total of 1676 contigs (4.128 MB) aligned with the NTUH-K2044 chromosome representing $78.7 \%$ genome coverage. Similarly, 1668 contigs (4.098 MB) mapped to $77.1 \%$ of the MGH 78578 genome, which includes two contigs mapping to the toxin-antitoxin system of the pKPN4 plasmid. We identified no additional sequence homology to K. pneumoniae plasmids, and the homologous toxin-antitoxin system is maintained on the chromosome for the NTUH-K2044 strain, suggesting that K. pneumoniae strain ATCC 43816 does not possess plasmids.

A high degree of genetic conservation was observed between the three $K$. pneumoniae strains with $96.3 \%$ of ATCC 43816 sequence mapping to both MGH 78578 and NTUH-K2044. However, $69.71 \mathrm{~kb}$ of ATCC 43816 contigs uniquely map with the NTUH-K2044 genome and $44.67 \mathrm{~kb}$ with the MGH 78578 genome 
( $1.66 \%$ and $1.06 \%$ total sequence data, respectively). While MGH 78578 shares primarily metabolic and hypothetical proteins with ATCC 43816, NTUH-K2044 shares the virulence-associated yersiniabactin biosynthetic operon, a Type IV secretion system, an iron transport system, a CRISPR locus, and an acetonin catabolism locus (Table 2). Given the conservation of known virulence determinants between ATCC 43816 and NTUH-K2044, the data suggests that NTUH-K2044 may share the same virulence potential as ATCC 43816 in disease models. Conversely, MGH 78578 lacks several of the virulence determinants previously identified as critical to the disease potential of ATCC 43816 in murine respiratory disease models, suggesting that $\mathrm{MGH} 78578$ may have a reduced virulence in these models, though MGH 78578 is notably a clinical lung isolate from a presentation of pneumonia.

We also identified novel genetic sequences unique to ATCC 43816, and not present in either NTUH-K2044 or MGH 78578, notably including two bacteriophages, one of which is homologous to a bacteriophage found in the enteric Escherichia coli strain UMN026, and the other to the upper respiratory tract (URT) pathogen Klebsiella rhinoscleromatis strain ATCC 13884 (Table 2). Little has been reported regarding the clinical history of ATCC 43816, however the presence of both gastrointestinal (GI) and URT-related bacteriophages suggests that this $K$. pneumoniae strain may have previously been resident of both host niches.

Next Generation Sequencing provided an estimated $78 \%$ coverage of the ATCC 43816 genome, however sequencing of the capsular polysaccharide 
biosynthetic locus was under-represented at $22 \%$ coverage (determined retrospectively). Given the importance of capsule as a virulence determinant, we completed sequencing of a $34.6 \mathrm{~kb}$ region which includes the capsule locus as well as an adjacent region ( $w z m$ to $w b b O$ ) reported to be required for LPS biosynthesis (Figure 3) [209]. The K2 capsular polysaccharide locus shares broad homology to sequenced capsule loci from the galF to $w z c$ and $r f b P$ to uge genes, but shares specific homology over the central orf7 to orf13 genes to a subset of sequenced K. pneumoniae strains of K2 serotype. Homology over the entire capsule locus is therefore highest ( $99 \%$ identity) to that of the recently fully sequenced strains CG43 (Accession CP006648), KCTC 2242 (CP002910), and Kp52.145 (FO834906), and also to the partially sequenced capsule biosynthetic loci of VGH525 (Accession AB371296) and Chedid (D21242). The central region of the capsule loci encodes for the antigenic diversity of capsules, which has previously supported the use of PCR as a methodology to identify capsule serotype $[210,211]$.

\subsubsection{Characterization of capsule production}

K. pneumoniae capsular serotypes influence the virulence potential of strains, as could the regulated production of capsule. Thus, we investigated whether differences exist in the amount of capsule produced by the three clinical strains examined in this study. K. pneumoniae were examined for their ability to produce capsule from LB overnight cultures. We found that ATCC 43816, NTUH-K2044 and MGH 78578 strains produced 9.7, 13.5, and $6.1 \mathrm{fg} / \mathrm{CFU}$ of 
capsule, respectively. As a control, we also measured capsule production from previously characterized strain 52.145 which produced $21.7 \mathrm{fg} / \mathrm{CFU}$ of capsule in our studies, consistent with 52.145 being a significant producer of capsule [182]. Our measurement of NTUH-K2044 capsule production is consistent with previously reported levels of $17.3 \mathrm{fg} / \mathrm{CFU}$ of capsule from overnight LB cultures [212]. Thus, of the sequenced strains studied in this work, NTUH-K2044 produced the greatest amount of capsule at levels 1.4 and 2.2 fold greater than ATCC 43816 and MGH 78578. Similar amounts of capsule were measured from strains grown to mid-exponential phase in TSBDC, suggesting that media and growth phase do not significantly impact capsule production.

\subsubsection{Cell culture model}

K. pneumoniae is internalized by a variety of host cell types both in vivo and in cell culture models, thus we investigated whether the three study strains exhibit differences in uptake rates in cultured murine macrophages. Given that capsular polysaccharide has been reported to mediate an antiphagocytic phenotype, we also generated capsular polysaccharide mutants of ATCC 43816 $(\Delta \operatorname{man} C)$ and NTUH-K2044 $(\Delta w Z C)$, and confirmed that the capsule mutants exhibited reduced exclusion of nigrosin staining (Figure 4). Both J774A.1 and RAW264.7 monolayers were challenged with $K$. pneumoniae strains at an MOI of 10 , and internalized bacteria were detected using a gentamicin protection assay. In both J774A.1 and RAW264.7 cells, the MGH 78578 strain was phagocytosed more efficiently than the ATCC 43816 and NTUH-K2044 strains $(P<0.001)$ 
(Figure 4). This suggests that the K52 serotype capsule of the MGH 78578 strain does not resist uptake by murine macrophages to the same degree as representative $\mathrm{K} 1$ and $\mathrm{K} 2$ serotype strains. In addition, ATCC 43816 was phagocytosed more efficiently than NTUH-K2044 in both cell lines $(P<0.001)$, suggesting that there may be differences in the antiphagocytic properties of K1 and K2 capsular polysaccharides or other surface exposed factors.

We therefore also investigated whether capsule alone mediates the antiphagocytic phenotype by comparing ATCC 43816 and NTUH-K2044 capsule mutants to relatively highly phagocytosed MGH 78578. Both ATCC $\triangle \operatorname{manC}$ and NTUH $\triangle W Z C$ capsule mutants were phagocytosed at significantly higher rates than their isogenic wild type parent strains in both cell lines (Figure 5), and furthermore, the NTUH $\triangle W Z C$ was significantly less phagocytosed than the MGH 78578 strain. These data demonstrate that the NTUH $\Delta w z C$ capsule mutant retains antiphagocytic properties which are distinct from capsular polysaccharide, suggesting that additional factors additionally mediate the antiphagocytic phenotype of $K$. pneumoniae. The ATCC $\triangle \operatorname{manC}$ was phagocytosed at levels similar to the MGH $78578 \mathrm{~K} 52$ strain, and it is therefore not possible to conclude whether ATCC 43816 possesses non-capsule antiphagocytic determinants using the K52 MGH 78578 strain which may itself be antiphagocytic. Due to the intrinsic antibiotic resistance of the MGH 78578 strain to common antibiotic markers used for molecular biology, we were unable to generate an acapsular MGH 78578 strain for these studies. 
We decided to investigate whether K. pneumoniae is replicationcompetent within cultured macrophages after internalization. Both RAW264.7 and J774A.1 murine macrophages were infected at an MOI of 10 with subsequent evaluation of bacterial colonization at time points $3,4.5,6,9$ and 12 hr post infection. As observed previously, MGH 78578 was internalized at the highest levels in both cell lines, while NTUH-K2044 had the lowest level of internalization at the $3 \mathrm{hr}$ time point (Figure 6). Proliferation of $K$. pneumoniae was observed for all bacterial strains, however during $9 \mathrm{hr}$ of observation between the 3 and $12 \mathrm{hr}$ time points the average fold increase in bacterial number was just 3.0-4.6 fold in J774A.1 cells. Higher rates of proliferation were observed for ATCC 43816 and MGH 78578 in RAW264.7 cells, however NTUHK2044 saw only a 2.9 fold increase in bacterial numbers from $3 \mathrm{hr}$ to $12 \mathrm{hr}$.

These data indicate that the K1 (NTUH-K2044) and K2 (ATCC 43816) strains of K. pneumoniae are internalized at relatively low rates but are replication competent in cultured macrophages. However, the K52 strain, MGH 78578, was phagocytosed at relatively high levels in both cell lines, and proliferated significantly in RAW264.7 cells. Importantly, intracellular ATCC 43816 and MGH 78578 exhibited significant outgrowth in cultured macrophages between the 3 and $12 \mathrm{hr}$ time points (both J774A. 1 and RAW264.7, P<0.001), indicating that $K$. pneumoniae possesses replicative viability within macrophages, in spite of its classification as an extracellular pathogen. These data suggest that differences between K. pneumoniae serotype, and potentially other genetic determinants, 
could impact the preferred host niche during disease, including the propensity to persist within macrophages.

\subsubsection{Respiratory murine model of $K$. pneumoniae infection}

We decided to investigate the virulence potential of the three sequenced K. pneumoniae strains in a murine respiratory disease model. Because $K$. pneumoniae is known to colonize the upper respiratory tract (URT) in mammals, we developed a novel infection model to deliver $K$. pneumoniae non-surgically into the lung of mice using intubation-mediated intratracheal (IMIT) instillation, specifically modeling lower respiratory tract (LRT) disease. Female BALB/c mice

were challenged with one of the three $K$. pneumoniae study strains using the IMIT infection method using multiple challenge doses to estimate the $50 \%$ lethal dose (LD50). Both ATCC 43816 and NTUH-K2044 were found to be highly virulent strains of $K$. pneumoniae $\left(\mathrm{LD}_{50}<100\right)$ while the MGH 78578 strain is significantly less virulent, with an $\mathrm{LD}_{50}>10^{5.4}$ fold higher than the virulent strains (Table 3). K. pneumoniae respiratory disease in the IMIT mouse model is associated with an acute course of disease with minimally lethal doses resulting in moribund disease within 3-4 days (Figure 7).

Groups of mice infected with minimally lethal doses of ATCC 43816 and NTUH-K2044 (102.2 CFU) were necropsied to investigate bacterial burdens in host tissues. Bacteria were enumerated by plate count from blood, and homogenates of lung, liver, and spleen. Moribund mice were found to have the highest levels of host colonization in both blood and lung, followed by liver and 
spleen (Figure 8). One-way ANOVA with Tukey Post Test revealed no significant difference between ATCC 43816 and NTUH-K2044 bacterial burdens in any of the host samples examined. These data reveal that both ATCC 43816 and NTUH-K2044 K. pneumoniae pneumonia is associated with development of a significant bacteremia and systemic spread to multiple organs.

\subsection{Discussion}

K. pneumoniae respiratory infections may result from several mechanisms of pathogen introduction into susceptible hosts including inhalation of environmental sources of bacteria, as it relates to community acquired pneumonia (CAP), or nosocomial foreign body introduction of $K$. pneumoniae into the respiratory system, as in ventilator associated pneumonia (VAP). Thus, respiratory infections with K. pneumoniae are clinically important both to nosocomial pneumonia in hospital settings as well as CAP in developing areas of the world. The primary focus of this work was to gain insight into the molecular mechanisms which contribute to the respiratory disease caused by virulent $K$. pneumoniae. We therefore sequenced one of the commonly researched strains, capable of causing pneumonia in surrogate animal models. The ATCC 43816 strain was successfully sequenced by Next Generation approaches at approximately $80 \%$ coverage, based on estimated total sequence data relative to the NTUH-K2044 genome size. The majority of ATCC 43816 sequence (>96\%) was well conserved to both the highly virulent NTUH-K2044 and the minimally virulent MGH78578 strains suggesting that $K$. pneumoniae strains may have 
large core genomes, and that key differences in virulence potential may be related to a small number of pathogenicity islands.

Based on the data from our murine respiratory disease model, we identified NTUH-K2044 and ATCC 43816 as highly virulent K. pneumoniae strains, and MGH 78578 as a low virulence strain. These findings are consistent with clinical evidence that $\mathrm{K} 1$ and $\mathrm{K} 2$ serotypes of $K$. pneumoniae are most commonly associated with severe disease presentations, including CAP, invasive presentations, as well as lethality in a mouse intravenous challenge model [192, 213]. To the best of our knowledge, this current study provides the first experimental evidence demonstrating that a representative $\mathrm{K} 52$ clinical isolate is relatively avirulent in a murine respiratory disease model. Thus, phenotypic evidence links the newly sequenced ATCC 43816 strain to the fully sequenced NTUH-K2044 rather than MGH 78578 strain. The genetic systems found to be shared between NTUH-K2044 and ATCC 43816, but absent from MGH 78578 included iron acquisition genes (yersiniabactin biosynthesis and iron transport), a Type 4 secretion system (T4SS), a CRISPR locus, and an acetonin catabolism locus. Yersiniabactin biosynthetic operon has been previously demonstrated to be important to the function of ATCC 43816 in a murine intranasal respiratory disease model $[92,159]$, where yersiniabactin is thought to contribute to evasion of the activity of lipocalin2 in the lung - a host factor which neutralizes enterobactin-based iron acquisition [114]. The T4SS present in the virulent ATCC 43816 and NTUH-K2044 strains has been proposed to potentially represent a DNA conjugation system, and is also present in the related $K$. 
variicola environmental isolate strain $342[214,215]$, thus future studies will be required to investigate the virulence potential of this secretion system in $K$. pneumoniae. This study supports previous findings that the capsular serotype and the presence of the yersiniabactin iron acquisition systems contribute significantly to the disease potential of highly virulent $K$. pneumoniae strains, with the additional possibility that T4SS or acetonin catabolism may be important for K. pneumoniae disease.

In this study, we investigated whether there were any significant differences in the ability of $K$. pneumoniae strains to persist within cultured macrophages. K. pneumoniae is considered to be primarily an extracellular pathogen, although there is evidence that this organism may be internalized in human epithelial cell lines $[216,217]$. Furthermore, $K$. pneumoniae can be taken up into cultured murine peritoneal macrophages, and are also internalized in vivo in alveolar macrophages [95]. However, capsular polysaccharide may mediate blocking the initial attachment of bacteria to cells, reducing internalization [218]. Given the importance of capsule in mediating the uptake of $K$. pneumoniae into phagocytic cells, we anticipated that the representative K2 strain in our study, ATCC 43816, may resist uptake into cultured macrophages given the previous discovery that the K2 serotype is associated with an absence of mannose residues in the capsular polysaccharide [139]. Similarly, the gmd and wcaG genes, present in $\mathrm{K} 1$ serotype capsular loci, are required for the modification of mannose to fucose, and a corresponding low level of mannose/high level fucose in K. pneumoniae isolates which possess these genes [219]. We had observed 
that the K1 strain NTUH-K2044 exhibited particularly low uptake into cultured murine macrophages in our studies, and that the K2 strain ATCC 43816 also had a lower level of uptake than the K52 strain MGH78578. Similarly, capsule has been demonstrated to mediate anti-phagocytosis in both amoeba and alveolar macrophages, suggesting that this role for capsule is ubiquitously important across a range of host-pathogen interactions with professional phagocytes [203, 220]. Given the potential role of the mannose receptor in facilitating phagocytosis, and the reduced level of mannose residues in $\mathrm{K} 1$ and $\mathrm{K} 2$ serotype capsules, it is possible that modulation of the polysaccharide surface of $K$. pneumoniae may represent an important strategy for evading host defense.

Consistent with prior studies, we found that acapsular K. pneumoniae mutants also exhibited increased uptake into macrophages, however, we also observed that the increase in uptake of the NTUH-K2044 capsule mutant did not achieve the level of uptake of the representative K52 serotype strain. This finding suggests that the NTUH-K2044 strain possesses additional non-capsule antiphagocytic factors, or that the MGH78578 strain actively promotes its uptake into macrophages. Given that K. pneumoniae capsule does possess antiphagocytic properties as a strategy to act primarily as an extracellular pathogen, we hypothesize that the K52 MGH 78578 strain does not promote its own uptake, and instead, we hypothesize that the function of K52 capsules would be as an antiphagocytic determinant consistent with other serotype capsules. Thus, we interpret that the NTUH-K2044 strain possesses multiple mechanisms to resist phagocytosis, which is consistent with previous findings that capsule, 
LPS, carnitine metabolism, and the ClpX protease are all required to resist entry into amoeba and human neutrophils [220]. We conclude that $K$. pneumoniae antiphagocytosis is mediated by a multifactorial process and that capsule alone is insufficient to account for this phenotype. This conclusion is consistent with prior published findings that additional genetic loci participate in mediating $K$. pneumoniae antiphagocytosis, including genes for LPS, the ClpX protease, and carnitine metabolism [220].

In our respiratory challenge studies, the $\mathrm{K} 1$ and $\mathrm{K} 2$ serotype strains possessed a significantly higher virulence in mice than the K52 serotype strain, supporting the possibility that evasion of phagocytosis is a strategy employed by K. pneumoniae to enhance virulence in mammalian hosts. Thus, these finding support the characterization of $K$. pneumoniae as an extracellular pathogen, whereby the most virulent strains are also the most antiphagocytic. These results are consistent with previous reports which have demonstrated that antiphagocytic properties of $K$. pneumoniae capsule are associated with highly virulent K1 and K2 serotypes in panels of Asian strain isolates [131, 140]. It is however noteworthy that several additional virulence-associated genetic determinants are common to the $\mathrm{K} 1$ and $\mathrm{K} 2$ representative strains investigated in this study, including mechanisms of iron acquisition, highlighting that $K$. pneumoniae serotype is not the sole distinguishing factor for predicting disease potential in mammalian hosts.

Our data supports a link between evasion of phagocytosis and virulence potential as an interpretation that $K$. pneumoniae is primarily an extracellular 
pathogen. However, we interestingly demonstrate that $K$. pneumoniae strains are fit to replicate within macrophages once internalized. This is an intriguing finding that may suggest that a subpopulation of $K$. pneumoniae may use an intracellular lifestyle as part of the disease process. Future studies will be required to characterize to what extent $K$. pneumoniae has adapted to intracellular host-pathogen interaction, and what role the intracellular lifestyle might play in vivo.

We developed a novel respiratory disease model to study K. pneumoniae pneumonia which facilitated an investigation of the lower respiratory tract (LRT) colonization in the absence of the involvement of other primary sites of infection. Our motivation to develop this model system was in part shaped by our observations that bacterial pathogens may opportunistically infect the upper respiratory tract (URT) in a process which is unique from that observed during human disease [221]. Direct non-surgical instillation directly into the LRT also directly mimics a normal route of nosocomial acquisition of K. pneumoniae associated with VAP. Our novel intubation-mediated intratracheal (IMIT) delivery of bacteria facilitates: i) the use of a flow meter to validate the placement of a catheter into the trachea, ii) the ability to instill bacteria directly into the lung via a blunt needle inserted through the catheter lumen, iii) delivery of bacteria into the lower lobes of the lung under positive pressure, and iv) minimal trauma to the host owing to the non-surgical nature of the procedure.

We found that our novel IMIT infection model lowered previously reported LD50 values for both i.n. and surgical i.t. infection models, suggesting that 
targeted delivery of a reduced number of bacteria to the lungs enhances the disease potential of $K$. pneumoniae. The $\mathrm{LD}_{50}$ for the ATCC 43816 strain in the IMIT model is $10^{1.8}-10^{2.8}$ fold less than previously published LD50 values using intranasal models [92, 222], suggesting that the IMIT model provides a lower $\mathrm{LD}_{50}$ due to the ability of the intubation tubing to briefly occlude the air space, allowing for positive pressure delivery of organisms deep into the lung, rather than passive delivery into the lung in shallowly breathing anesthetized animals. Thus, our IMIT model improves the disease potential of $K$. pneumoniae in lungspecific disease, and may be an excellent instillation method for therapeutic and diagnostic reagents in studies requiring LRT delivery.

In summary, our study has provided a first draft sequence of the ATCC 43816 genome which will support future investigations of $K$. pneumoniae function. We also have provided the first description of the growth potential of $K$. pneumoniae in cultured murine macrophages, supporting a growing body of evidence that K. pneumoniae may not be exclusively an extracellular pathogen. Finally, we have developed and employed a novel non-surgical, lung-specific infection model which allows for targeted low dose inoculation of $K$. pneumoniae giving rise to a lethal pneumonia in mice, and revealing a significant difference in the disease potential of clinical isolates. 
Table 2. Genetic elements common/unique to sequenced strains

\begin{tabular}{|c|c|}
\hline $\begin{array}{l}\text { Genetic loci common to NTUH- } \\
\text { K2044 }\end{array}$ & Genes mapped (KP1_) \\
\hline Yersiniabactin biosynthesis & $\begin{array}{l}\text { 3583-3586, 3588-3593, 3605-3609, } \\
3611-3613\end{array}$ \\
\hline Type IV Secretion & $3634,3638-3641,3643$ \\
\hline Iron transport & 1980-1989 \\
\hline CRISPR locus & 3164-3166, 3171 \\
\hline Acetonin catabolism & $1112-1121$ \\
\hline Conserved and hypothetical genes & $\begin{array}{l}2362-2364,2378-2385,3239-3240, \\
3773\end{array}$ \\
\hline $\begin{array}{l}\text { Genetic loci common to MGH } \\
78578\end{array}$ & Genes mapped (KPN_) \\
\hline Metabolic & $\begin{array}{l}00033-00034,00594-00598,03359- \\
03370,03372,04612-04613\end{array}$ \\
\hline Conserved and hypothetical genes & $\begin{array}{l}\text { 01146-01148, 01151-01164, 01432- } \\
01433,01316-01317,04518\end{array}$ \\
\hline $\begin{array}{l}\text { ATCC } 43816 \text { genetic loci absent } \\
\text { from NTUH-K2044 and MGH } 78578\end{array}$ & Genes \\
\hline $\begin{array}{l}\text { Escherichia coli UMN026-like } \\
\text { bacteriophage }\end{array}$ & $\begin{array}{l}\text { ECUMN_0964-0965, 0975, 0977-0986, } \\
\text { 0994-0996 }\end{array}$ \\
\hline $\begin{array}{l}\text { Klebsiella pneumoniae subsp. } \\
\text { rhinoscleromatis ATCC 13884-like } \\
\text { bacteriophage }\end{array}$ & HMPREF0484_4775, 4777-4779, 1182 \\
\hline $\begin{array}{l}\text { Enterobacter radicincitans DSM } \\
16656 \text { acriflavine resistance }\end{array}$ & Y71_5381-5385 \\
\hline $\begin{array}{l}\text { Klebsiella oxytoca 10-5243 fimbrial } \\
\text { biosynthesis }\end{array}$ & HMPREF9687_02419-02420 \\
\hline $\begin{array}{l}\text { Hypothetical protein Salmonella } \\
\text { enterica subsp. enterica serovar } \\
\text { Saintpaul str. SARA29 }\end{array}$ & SeSPB_A4698 \\
\hline $\begin{array}{l}\text { Hypothetical protein Enterobacter } \\
\text { hormaechei ATCC } 49162\end{array}$ & HMPREF9086_3347 \\
\hline $\begin{array}{l}\text { Hypothetical protein Klebsiella sp. } \\
\text { 4_1_44FAA }\end{array}$ & HMPREF1024_04074 \\
\hline $\begin{array}{l}\text { Hypothetical protein Escherichia } \\
\text { hermannii NBRC } 105704\end{array}$ & EH105704_01_06400 \\
\hline
\end{tabular}


Table 3. Probit analysis of IMIT-infections of BALB/c mouse using $K$. pneumoniae strains

\begin{tabular}{|c|c|}
\hline Strain & LD $_{50}\left(95 \% \mathrm{Cl}^{\star}\right)$ \\
\hline ATCC 43816 & $4.71 \times 10^{1}\left(1.36 \times 10^{1}-1.63 \times 10^{2}\right)$ \\
\hline NTUH-K2044 & $2.33 \times 10^{1}\left(5.4 \times 10^{0}-1.01 \times 10^{2}\right)$ \\
\hline MGH 78578 & $1.37 \times 10^{7}\left(9.50 \times 10^{6}-1.97 \times 10^{7}\right)$ \\
\hline
\end{tabular}

* $95 \%$ confidence interval. 

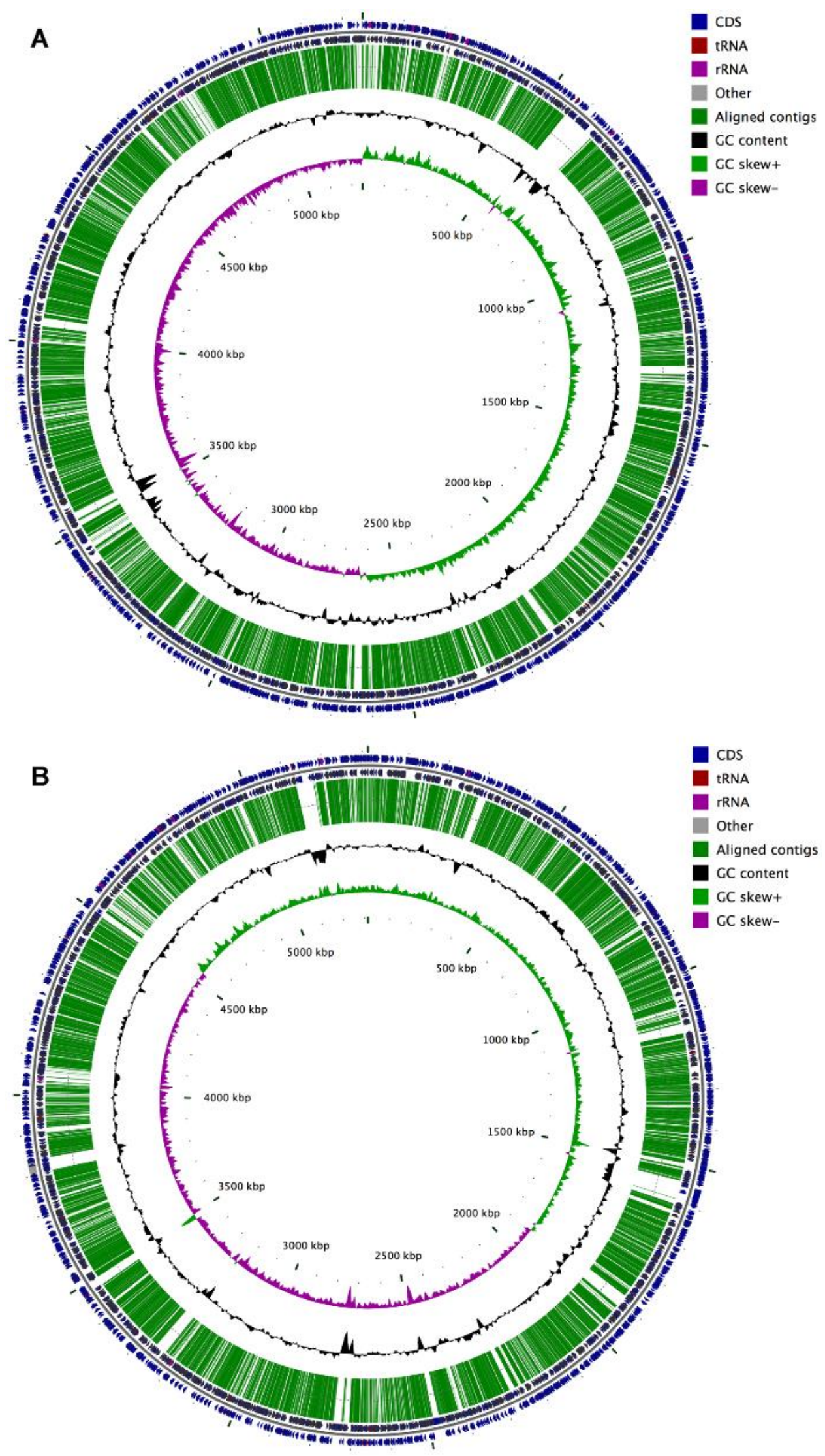
Figure 2. Alignment of the ATCC 43816 sequence to previously sequenced K. pneumoniae chromosomes. Next generation sequencing of ATCC 43816 produced 1763 contigs which were aligned to published NTUH-K2044 (Panel A) and MGH 78578 (Panel B) chromosomes. The locations of the contigs are shown as long green lines. The two outer tracks depict the annotated genes in the reference genomes (on both strands) while the two inner tracks show GC content and GC skew, respectively which strongly effects sequencing as can be seen from the coincidence of low GC content regions and gap in the contig alignments. 


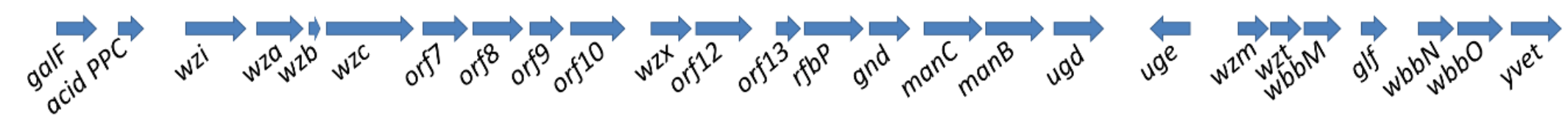

Figure 3. Genetic organization of the ATCC 43816 K2 capsule locus. Scale representation of the capsular polysaccharide biosynthetic locus (galF-uge) and LPS locus (wzm-yvet). The central domain of the capsule locus (orf7-orf13) represents the antigenic diversity region unique to K2 serotype capsules, while the remainder of the locus is well conserved with other $K$. pneumoniae capsule loci. 

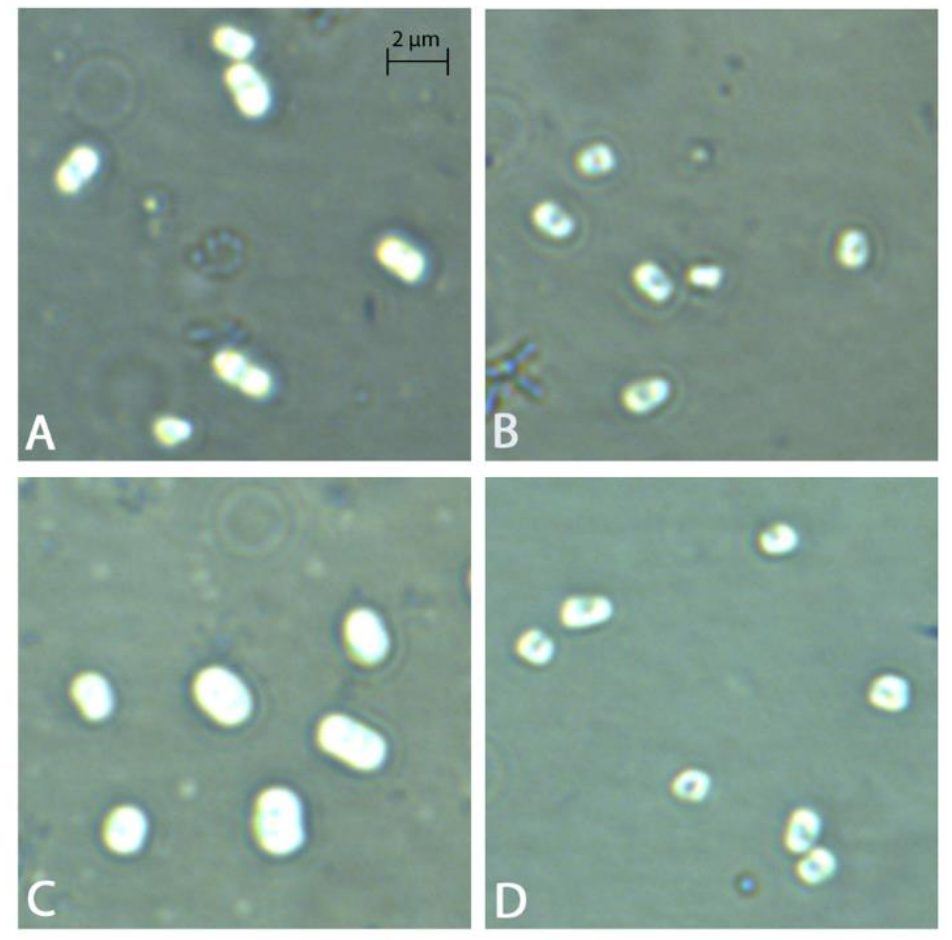

Figure 4. Negative staining of capsular polysaccharide from ATCC 43816 and NTUH-K2044 strains. Overnight bacterial suspensions of ATCC 43816 (A), ATCC $\Delta \operatorname{manC}(\mathrm{B}), \mathrm{NTUH}-\mathrm{K} 2044$ (C) and NTUH $\Delta w z c(\mathrm{D})$ were mixed in 1:1 ratio with $10 \%$ nigrosin. The loss of the capsular polysaccharide from the mutant strains was illustrated by the decrease exclusion of the nigrosin dye which was visualized with Zeiss Axio microscope (63x magnification). Imaris analysis identified that the cross-sectional areas of ATCC $\Delta \operatorname{manC}$, and NTUH $\Delta$ wzC mutants were reduced by $36.21 \%$, and $28.59 \%$, relative to their isogenic parents. 

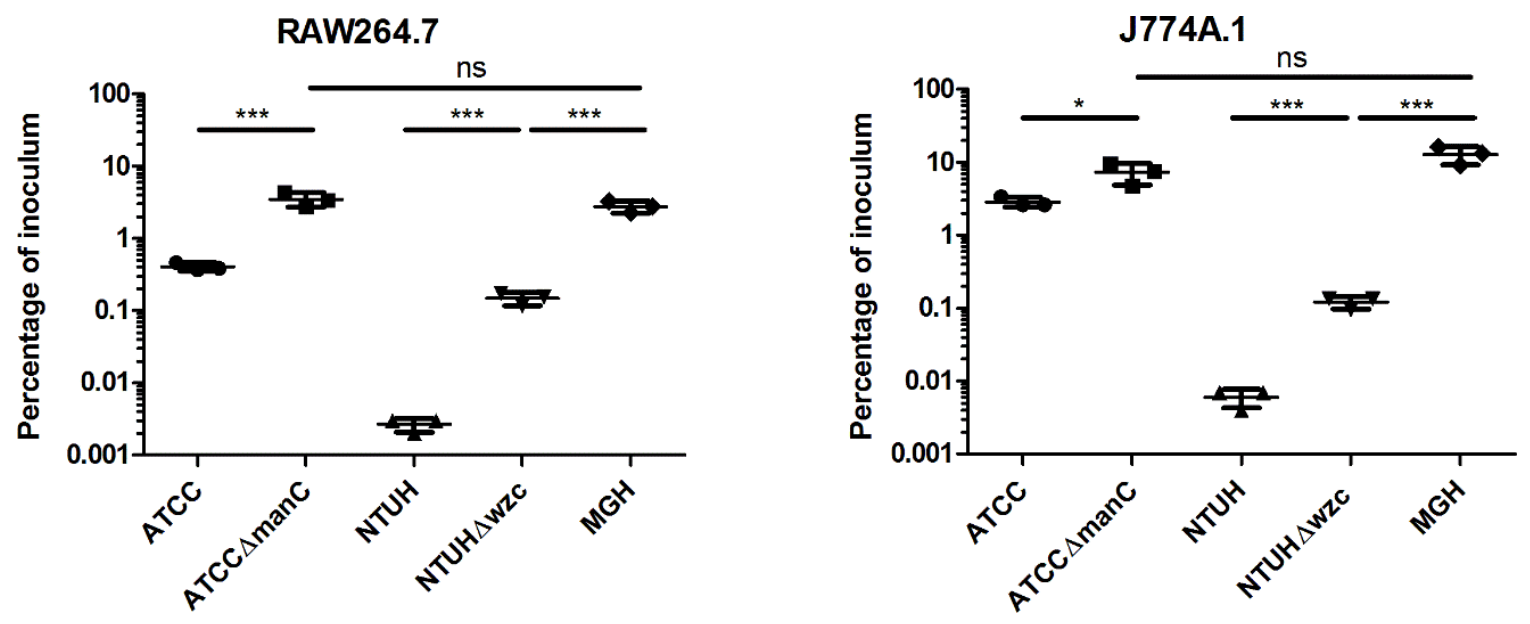

Figure 5. Uptake of K. pneumoniae wild type and capsular polysaccharide mutants strains into cultured murine macrophages. $K$. pneumoniae strains ATCC 43816 (K2), NTUH-K2044 (K1) and MGH 78578 (K52) or capsule mutants ATCC $\triangle m a n C$, and NTUH $\triangle W Z C$ were incubated in the presence of cultured murine macrophage cell lines J774A.1 or RAW264.7 at an MOI of 10 in 96 well plates. At one hour post-infection, gentamicin was introduced to eradicate extracellular bacteria, and uptake of $K$. pneumoniae strains into macrophages was assessed at $3 \mathrm{hr}$ post-infection by plate counting. Triplicate samples were enumerated and data analyzed as a percentage of the inoculum, with the results representative of at least two independent trials. 

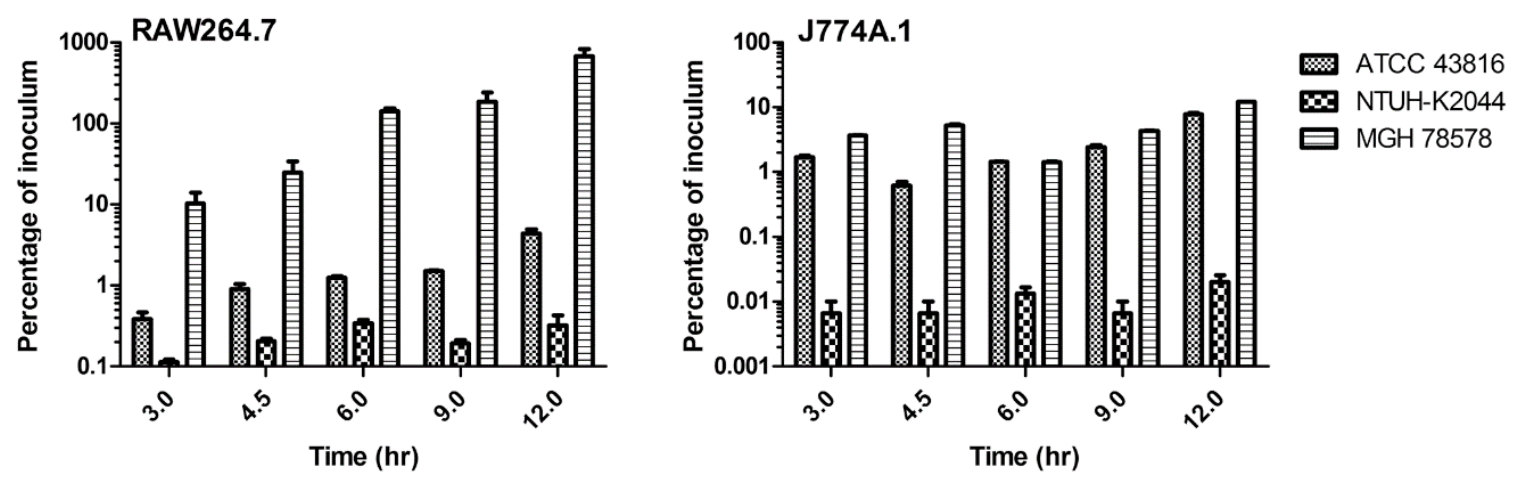

Figure 6. Growth potential of $K$. pneumoniae strains in cultured murine

macrophages. K. pneumoniae strains ATCC 43816 (K2), NTUH-K2044 (K1) and MGH 78578 (K52) were incubated in the presence of cultured murine macrophage cell lines J774A.1 or RAW264.7 at an MOI of 10 in 96 well plates. At one hour post-infection, gentamicin was introduced to eradicate extracellular bacteria. At $3,4.5,6,9$, and $12 \mathrm{hr}$ post-infection, a triplicate set of samples was processed for enumeration of intracellular bacteria. The data is representative of at least three independent trials. 

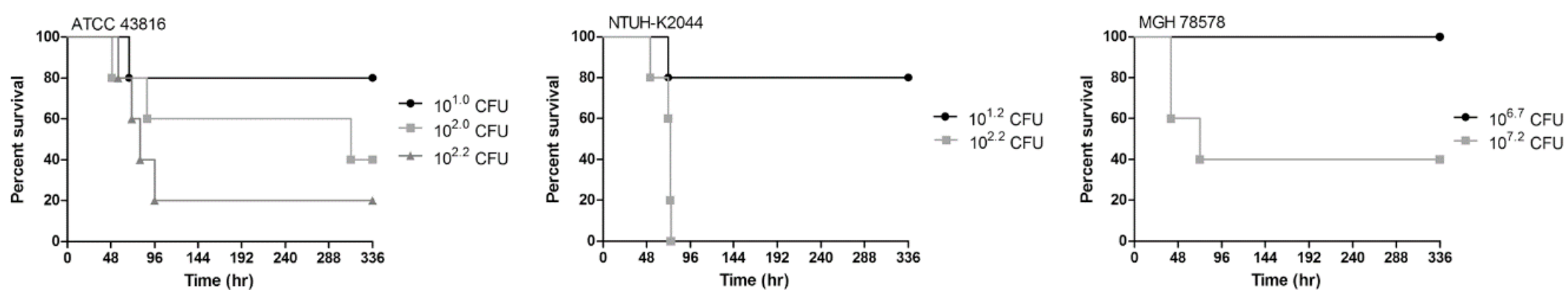

Figure 7. Survival analysis of $K$. pneumoniae respiratory challenge. Groups of five female BALB/c mice were challenged with K. pneumoniae strains ATCC 43816 (K2), NTUH-K2044 (K1) or MGH 78578 (K52) by IMIT respiratory infection. Mice were monitored for 14 days, and moribund mice were euthanized. The challenge dose for each survival curve group is indicated. 


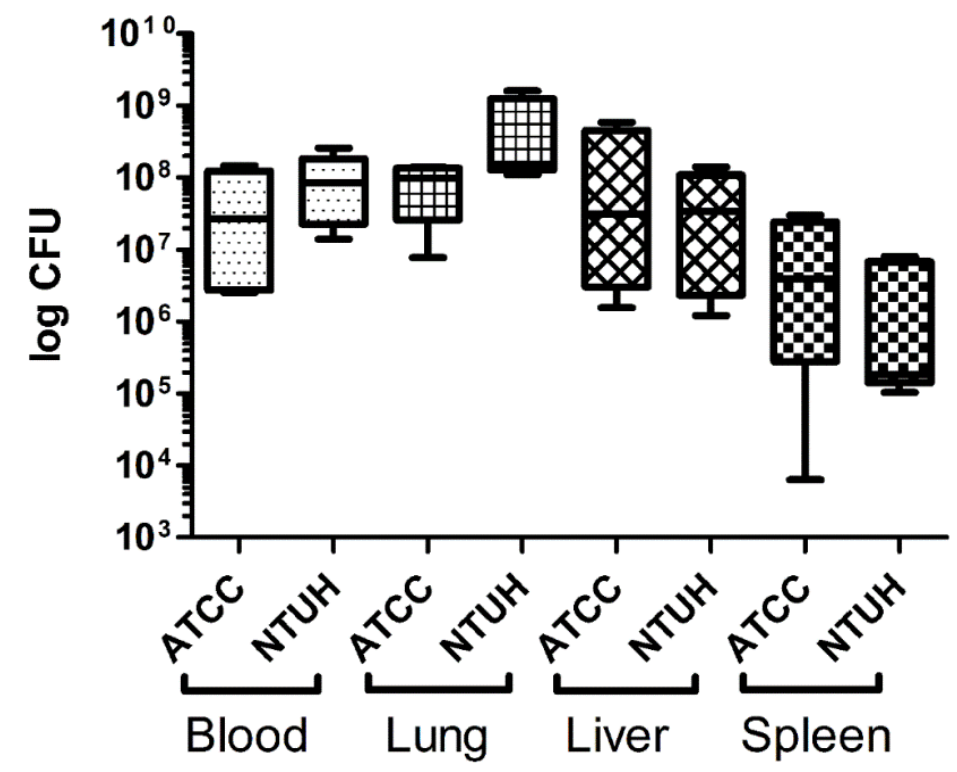

Figure 8. Bacterial burden of K. pneumoniae-infected mice. Groups of five female BALB/c mice were infected with K. pneumoniae strains ATCC 43816 or NTUH-K2044 and tissues were harvested at the onset of moribund disease, and homogenized in $1 \mathrm{ml}$ of PBS. Bacteria were enumerated from blood and from homogenates of lung, liver, and spleen. The results present a min/max box and whisker plot for each infected tissue $(n=5)$. Statistical analysis was carried out by one way ANOVA and Tukey post test. 
CHAPTER 4

\author{
DIRECT MONITORING OF MEROPENEM THERAPEUTIC EFFICACY \\ AGAINST KLEBSIELLA PNEUMONIAE RESPIRATORY INFECTION USING \\ OPTICAL DIAGNOSTIC IMAGING
}

\title{
4.1 Introduction
}

Klebsiella pneumoniae ssp. pneumoniae (K. pneumoniae) is a Gramnegative bacteria (GNB) ubiquitously present in the environment, and can also be cultured from host mucosal surfaces, and medical devices, where it excels as a nosocomial pathogen $[14,188,223]$. As an opportunistic pathogen, $K$. pneumoniae causes community and hospital acquired infections with more cases being isolated from the latter. K. pneumoniae is a causative agent for multiple nosocomial diseases including urinary tract infection, septicemia, pneumonia, and premature infant infections in the intensive care unit [224-228]. $\mathrm{K}$. pneumoniae is a significant contributor to hospital acquired pneumonia accounting for $25-43 \%$ of Gram-negative nosocomial respiratory infections [229]. The pneumonic disease is characterized by a rapid progressive clinical course leading to multilobar involvement, abscess formation, and systemic spread of the bacteria with severe outcomes leaving little time for therapeutic intervention [95, 230-233]. Populations at higher risk of acquiring the respiratory disease include 
neonates, immunocompromised individuals, and people with underlying diseases $[35,234,235]$. The mortality rates associated with the pneumonic disease are quite high approaching or exceeding $50 \%$ even with antibiotic treatment $[80,236$, 237].

There is a growing concern about the emergence of multidrug resistant (MDR) K. pneumoniae strains such as the extended-spectrum beta-lactamases (ESBLs) and more recently the carbapenem resistant Klebsiella (KPC) which limit the treatment options with the current available therapeutics [238-242]. Therefore, there is an urgent need for the development of new antimicrobial agents to treat infections caused by this critical pathogen. In developing novel drugs, many therapeutic studies focus on monitoring host biometric endpoint/disease resolution criteria such as weight, temperature, and symptoms of disease to indirectly monitor therapeutic efficacy, while neglecting the direct effect of the drug on the pathogen itself. Therefore, to address this scientific gap we decided to generate a bioluminescent $K$. pneumoniae bioreporter strain in order to monitor noninvasively therapeutic efficacy in vivo while focusing our attention on microbial viability in real-time instead of inferring the drug efficacy from host physiology.

Bioluminescence imaging (BLI), an approach developed over the past decade, has been used to detect bioluminescent live cells within small animals [243]. The BLI involves the use of luminescent microorganisms to infect a host and monitor disease development using optical diagnostic imaging, and bacteria that do not naturally produce bioluminescence can be engineered to do so 
through inserting a luxCDABE operon into bacterial genome or by transforming the bacteria with a plasmid carrying the lux genes [244]. The lux operon encodes for the elements needed to generate a luciferase enzyme together with its substrate. In the presence of oxygen, luciferase catalyzes the breakdown of the substrate producing blue-green light as a byproduct [245]. BLI has been employed by different groups to study altered disease outcomes upon infecting animals with bacterial mutants [221, 246-248], to examine the change in dissemination patterns upon introducing the microbes through different routes of infection [246, 247], and to test antimicrobial agents efficacy in vivo [249-253]. There are multiple advantages for the use of BLI in biomedical research which include: 1) minimizing the number of animals needed to perform time course studies by eliminating the need to sacrifice animals at specific time points to enumerate tissue burdens, 2) eradicating the requirement for additional steps to estimate bacterial numbers in broth media or within host tissues reducing processing time and cost, 3) reducing individual-to-individual variations since bacterial viability is monitored within the same group of animals over the entire course of disease reducing error and increasing resolution, and 4) identifying microbial dissemination to unexpected host niches.

As one of the broad spectrum carbapenem antibiotics, meropenem has been demonstrated to be effective in treating patients with nosocomial pneumonia [254-256]. Meropenem is active against most clinically important Gram-negative pathogens of the lower respiratory tract including MDR Enterobacteriaceae [257-261]. Unlike imipenem, another drug from the 
carbapenem family, meropenem is more resistant to hydrolysis by the renal dehydropeptidase I (DHP-I), and therefore, meropenem does not require the additional administration of the DHP-I inhibitor cilastatin [262, 263]. Meropenem is effective in penetrating respiratory tract tissues [264], making it a good drug for treating infections at that anatomical site. It is the recommended drug for treating pulmonary infections caused by K. pneumoniae especially for the MDR isolates $[43,265]$.

Meropenem was demonstrated to be effective against $K$. pneumoniae infections in mice including respiratory, blood, and thigh infections but with no calculation of the drug $50 \%$ effective dose (ED50) $[87,118,266,267]$. In those studies meropenem was used to treat MDR K. pneumoniae strains which typically require high bacterial inoculums in order to cause a respiratory infection in animals $[87,118]$. On the other hand, we have demonstrated that $K$. pneumoniae ATCC 43816 strain is capable of causing lethal pulmonary disease with a very low dose of the bacteria [99]. ATCC 43816 strain has also been used frequently in biomedical science investigations directed toward characterizing host response to the pulmonary disease $[111,113,115]$. In addition, we demonstrated that this strain is amenable to genetic manipulation [99], which makes it an excellent model pathogen suitable for conducing therapeutic studies against $K$. pneumoniae respiratory disease.

We decided to generate a bioluminescent reporter strain from ATCC 43816 to enable noninvasive monitoring of therapeutic efficacy of current clinically important drugs and future novel treatment approaches against $K$. 
pneumoniae respiratory disease. Furthermore, our knowledge about $K$. pneumoniae pathogenesis, virulence factors required during the respiratory disease and host-pathogen interaction is limited, and therefore, we decided to use the bioluminescent strain to facilitate such studies. The bioluminescent strain was utilized for direct monitoring of the viability of a manC capsule mutant, known to be attenuated during the pulmonary disease [92], to investigate whether BLI would enable monitoring the initiation of bacterial clearance from the lung. Finally, we have demonstrated previously that $K$. pneumoniae is capable of intracellular proliferation within host cells [99], and thus, the bioluminescent $K$. pneumoniae strain was employed to noninvasively monitor this intriguing phenotype to facilitate future investigations directed toward characterizing the importance of the intracellular lifestyle for K. pneumoniae during the respiratory disease.

\subsection{Results}

\subsubsection{Generation of bioluminescent $K$. pneumoniae bioreporter strain}

To begin to address the development of biodiagnostic imaging capability to monitor the therapeutic efficacy in vivo, we started by generating a bioluminescent strain. The bioluminescent K. pneumoniae strain (named JSKP001) was generated through the insertion of the luxCDABE operon into the chromosome of the strain ATCC 43816, commonly used to study host response to lung infection $[111,113,115]$. The lux operon was engineered to be under the control of the 50 s ribosomal subunit $r p / U$ promoter expected to be constitutively 
expressed in viable bacteria due to the requirement for protein synthesis in metabolically active microbes. The insertion of the lux operon took place between the convergently transcribed fus $A$ and yeeF genes which represent an insertional target for the nitrogen fixation operon of related Klebsiella spp., and therefore, the insertion in this site is predicted not to impact other genetic systems. However, the lux system requires energy from the cell which might impact bacterial growth potential.

To confirm that the insertion of the lux operon did not alter bacterial fitness, we tested the ability of the JSKP001 strain to proliferate in broth culture media relative to its parental strain. Overnight bacterial cultures in LB broth were diluted 1,000 fold and bacterial growth was monitored for $4 \mathrm{~h}$ by $\mathrm{OD}_{600}$ and bioluminescence. The JSKP001 had a doubling time similar to the ATCC parental strain, (estimated between $60-200 \mathrm{~min}$ ) of $20.83 \pm 0.49$ and $21.73 \pm 0.48$ min, respectively, which demonstrates that the knock-in mutagenesis did not affect the bacterial fitness in vitro (Figure 9A).

Further, to investigate the correlation between bacterial numbers and bioluminescence, overnight bacterial cultures were diluted 1,000 fold and grown in LB broth at $37^{\circ} \mathrm{C}$ with agitation for $3 \mathrm{hr}$, where bioluminescence and bacterial numbers were estimated at 30 min intervals. We had excellent correlation $\left(R^{2}=\right.$ 0.971) between bioluminescence and bacterial numbers (Figure 9B). Together, these data demonstrate that bioluminescence can be used to estimate bacterial numbers in broth culture media; the insertion of the lux operon into ATCC 43816 genome did not impact bacterial fitness. 


\subsubsection{Noninvasive tracking of respiratory disease}

We demonstrated that the lux operon acquisition of energy does not impact the ability of $K$. pneumoniae to grow in broth culture. However, this is an ideal and artificial situation where the bacteria is being provided with a rich source of nutrients; therefore, we decided to investigate if the insertion of the lux operon affected bacterial fitness in vivo where nutrients/energy availability is more limited. The ability of JSKP001 and ATCC 43816 strains to cause the pulmonary infection were compared where albino C57BL/6J mice were infected with lethal dose $\left(\sim 10^{4.9} \mathrm{CFU}\right)$ with either of the two bacterial strains using intubation-mediated intratracheal (IMIT) installation to enhance lung-specific delivery of pathogen $[99,187]$. Animals were euthanized upon meeting endpoint criteria consist of heart rate of 400 beat per minute $(\mathrm{bpm})$ or below in addition to demonstration of clear signs of illness including reduced mobility, decreased resistance upon handling, hunching, and shortness of breath (SOB). Infection with both ATCC 43816 and JSKP001 resulted in the development of the acute respiratory infection with no significant difference $(p=0.26)$ in the ability to cause a lethal moribund disease with median time to death (MTTD) of $\sim 3$ days (Figure 10A). This means that in addition to the demonstration that in vitro fitness of JSKP001 was not impacted by the insertion of the lux operon; the ability of the bacteria to cause lethal pulmonary infection was not altered as well.

We next decided to investigate the ability of the JSPK001 for systemic dissemination in comparison to its parental strain as a higher resolution approach 
of characterizing the impact of the insertion of the lux operon on the fitness of the bioluminescent strain. We looked at bacterial burdens in blood, BAL, lung, liver, spleen, and kidneys collected from animals at moribund disease. We observed similar bacterial burdens in the tissues collected from animals infected with JSKP001 and ATCC 43816 strains (Figure 10B). Collectively, we demonstrated that the insertion of the lux operon did not alter the virulence of the bioluminescent strain in our mouse lung-specific disease model and that JSKP001 is capable of disseminating and colonizing other tissues to wild type levels.

To have a better understanding of the virulence of JSKP001 and to facilitate future studies, we investigated the $50 \%$ lethal dose $\left(L D_{50}\right)$ for bioluminescent strain in the mice. Animals were infected with $10^{2}-10^{4.9} \mathrm{CFU}$ using IMIT, monitored for the development of the respiratory disease, and euthanized when moribund. The highest bacterial inoculum (104.9 CFU) caused $100 \%$ mortality while all animals survived the $10^{2} \mathrm{CFU}$ challenge (Figure 11 ). Using probit analysis, the JSKP001 LD 50 was calculated to be $10^{2.8} \mathrm{CFU}$ making the albino $\mathrm{C} 57 \mathrm{BL} / 6 \mathrm{~J}$ mouse model more resistant to the respiratory disease than BALB/c mice used in previous work [99].

We next investigated whether diagnostic imaging could be used to noninvasively monitor K. pneumoniae colonization of host lungs. Mice were infected with $125 x$ the LD 50 of the JSKP001 strain $\left(10^{4.9} \mathrm{CFU}\right)$ and bacterial colonization of host lungs as a function of bioluminescence were monitored twice daily using optical diagnostic imaging. Bioluminescence imaging (BLI) enabled 
real-time monitoring of bacterial colonization of mice lungs as well as bacterial dissemination to other anatomical sites (Figure 12A). Bioluminescence was detectable within mice thoracic cavity as early as $18 \mathrm{hr}$ post-infection and kept increasing throughout the time course of the disease (Figure 12B). Interestingly, even though the animals were infected with almost the exact amount of bacterial inoculum, knowing how effective IMIT is as a lung-specific delivery system [187], we observed $\sim 1.3$ log variation in thoracic cavity bioluminescence from the different animals (Figure 12B). Moreover, higher bacterial burdens at the $18 \mathrm{hr}$ time point correlated with earlier development of moribund disease with $R^{2}$ of 0.68 (Figure 12C). The variation in bioluminescence could be due to differences in light expression, and therefore, it is important to investigate whether bioluminescence correlate with bacterial numbers in vivo as we have demonstrated in broth media. Other explanations of such phenomena is that in some animals the bacteria might be going under early dissemination as observed in figure $12 \mathrm{~A}$, and thus, those animals end up with less bacteria in the lung. Another possibility might involve early eradication of bacteria by host immune system which is taking place in some animals but not others.

Together, we demonstrated that the genetic manipulation performed on the JSKP001 strain did not impact bacterial fitness in broth media and in vivo. We determined the $\mathrm{LD}_{50}$ for the JSKP001 strain and demonstrated that albino C57BL/6J mice are less susceptible to the respiratory disease than the previously used BALB/c model [99]. BLI allowed for noninvasive monitoring of the development of the respiratory disease in mice and revealed some possible 
interesting host-pathogen interactions. Finally, we have provided evidence that optical diagnostic imaging can facilitate earlier prediction of disease outcomes.

\subsubsection{Bioluminescence correlates with bacterial burdens}

To investigate the differences observed in thoracic cavity bioluminescence at early time point, we decided to test whether bioluminescence represent bacterial numbers within host tissues. Albino C57BL/6J mice were infected with JSKP001 $\left(10^{3}-10^{5.2} \mathrm{CFU}\right)$, monitored for the development of the respiratory disease, euthanized when moribund, and blood, BAL, lung, liver, spleen, and kidneys were collected for ex vivo bioluminescence measurement, and bacterial enumeration. Similar to the previous in vitro data, we had good correlations between bacterial numbers and bioluminescence where the $R^{2}$ values for blood, BAL, lung, liver, spleen, and kidneys were $0.69,0.47,0.57,0.81,0.53$, and 0.8 , respectively (Figure 13A-F).

Furthermore, we examined if bioluminescence can estimate the level of bacterial colonization within host thoracic cavity in life. Bioluminescence estimated at moribund disease right before euthanasia was compared with bacterial burdens in lung homogenates. Similarly, we had good correlation between the two measurements with an $R^{2}$ value of 0.55 (Figure 13G). These outcomes demonstrate that bioluminescence can be utilized to noninvasively estimate bacterial loads within host tissues. Together with the in vitro data, we demonstrated that the lux operon, under the control of the $r p / U$ promoter, is being constitutively expressed in broth media as well as within host tissues. 
Furthermore, we have demonstrated that the 1.3 log variation in bioluminescence observed from mice lungs at the $18 \mathrm{hr}$ time point (Figure 12B) was not due to differences in light expression but rather it might be due to differences in early bacterial trafficking or early clearance by host immune response.

\subsubsection{In vitro monitoring of bacterial viability using bioluminescence}

One benefit of having a bioluminescent $K$. pneumoniae strain is to enable the performance of high throughput in vitro assays for testing the efficacy of antimicrobial agents against this important pathogen. MicroChem-Plus is a quaternary multipurpose common detergent disinfectant designed for controlling the hazard of cross contamination of microbial pathogens, and it is broadly used in many facilities including hospitals and nursing homes. It is one of many antimicrobial agents and we decided to use this strong disinfectant to investigate whether bioluminescence can facilitate tracking bacterial viability over a short time course upon initiating the detergent treatment.

LB broth culture of exponentially growing JSKP001 strain were centrifuged, resuspended in PBS, and treated with serially diluted MicroChemPlus disinfectant where bacterial viability were monitored at room temperature by measuring bioluminescence at 1 min intervals and bacterial numbers were estimated following 11 min of incubation. Rapid drop in bioluminescence was observed within minutes after treatment initiation and CFU numbers estimated at 11 min were consistent with light emitted at that time point suggesting that the decrease in bioluminescence observed at earlier time points represent loss of 
bacterial viability (Figure 14A, and B). Slight decrease in bioluminescence was observed in the control group ( $0 \%$ MicroChem-Plus) which is probably due alteration in lux transcription levels caused by transferring the bacteria from rich broth (LB) into PBS. The use of MicroChem-Plus at the $2 \%$ manufacturer recommended concentration was able to kill the bacteria in less than a minute which was illustrated by the diminished bioluminescence consistent with the fact that the emission of bioluminescence requires continuous supply of energy provided by living microorganisms.

Furthermore, we have measured the correlation between bioluminescence and bacterial numbers at the 11 min time point, in sub-lethal concentrations of the disinfectant, and found out that the relationship between the two measurements are direct with $R^{2}$ value of 0.99 (Figure 14C), demonstrating that the loss of bioluminescence represent loss of viability and the transition from loss of bacterial viability to loss of light seems to be immediate. These outcomes demonstrate the power of the bioluminescent bioreporter tool to allow noninvasive viability assessments which are typically not possible using standard bacteriological approaches.

\subsubsection{In vitro tracking of bacterial growth inhibition by meropenem}

Having demonstrated that bioluminescence is a direct measurement of bacterial viability, we next decided to investigate whether we can use bioluminescence to monitor the impact of clinically important antibiotics such as meropenem on bacterial growth in vitro. Similar to other beta-lactam antibiotics, 
meropenem is bactericidal to many Gram-negative bacteria in which it inhibits bacterial cell wall synthesis. We utilized meropenem in a time course assay where an exponentially growing bacteria were mixed with serially diluted meropenem and bacterial growth was monitored by $\mathrm{OD}_{600}$ (Figure 15A) and bioluminescence (Figure 15B). Similar dose response of bacterial growth inhibition by the antibiotic was observed by both measurements (Figure $15 \mathrm{~A}$ and 15B).

Furthermore, meropenem $50 \%$ effective concentration $\left(E_{50}\right)$ calculated 8 hr post treatment using bioluminescence $(0.015 \mu \mathrm{g} / \mathrm{ml})$ was not significantly different $(p=0.059)$ from the EC 50 estimated by $\mathrm{OD}_{600}(0.023 \mu \mathrm{g} / \mathrm{ml})($ Figure 15C and 15D). Meropenem minimum inhibitory concentration (MIC) against JSK001 is estimated to be $0.125 \mu \mathrm{g} / \mathrm{ml}$, rendering this strain sensitive to the antibiotic. Together, these data demonstrate that bioluminescence can be used to monitor bacterial viability upon treating the microbes with an antibiotic and can also facilitate estimating antibiotics $\mathrm{EC}_{50}$ alternative to the traditionally used $\mathrm{OD}_{600}$ measurement, potentially with improved limit of detection.

\subsubsection{Noninvasive monitoring of meropenem therapeutic efficacy in mice}

Having demonstrated that using BLI we could monitor bacterial viability in vitro upon treating the microbes with antimicrobial agents, we decided to investigate if the same concept is applicable in vivo. We wanted to test whether we can use optical diagnostic imaging to monitor the efficacy of current and novel therapeutics noninvasively, and since we have demonstrated that the JSKP001 
strain is sensitive to meropenem, we decided to utilize this antibiotic as a proof of concept to investigate this question. Meropenem is an important drug for treating K. pneumoniae infections including pneumonia $[43,87,118,265-267]$. Therefore, we decided to investigate if meropenem is capable of resolving the respiratory disease and if the direct impact of the therapeutic on bacterial viability can be monitored in vivo using BLI.

To develop a working understanding of the preferable meropenem concentration for conducting the in vivo monitoring, we have performed survival studies and identified meropenem ED50 in our lung-specific respiratory disease model. Albino C57BL/6J mice were infected intratracheally with a lethal dose of JSKP001 (104.9 CFU), treated subcutaneously with increasing concentrations of meropenem antibiotic q12h (96 - $400 \mathrm{mg} / \mathrm{kg} /$ day), and euthanized upon reaching moribund disease (described earlier) or after completing 7 days of monitoring. Full protection from the respiratory disease was achieved with the $400 \mathrm{mg} / \mathrm{kg} / \mathrm{day}$ treatment, while less protection was observed the more we titrate the antibiotic (Figure 16A), and meropenem ED50 was estimated to be $221 \mathrm{mg} / \mathrm{kg} /$ day.

The development of the respiratory disease in the mice was monitored by measuring animals' heart rates and thoracic cavity bioluminescence. Heart rate was used previously to monitor progression of a respiratory infection caused by another pathogen where bradycardia was associated with bad prognosis [268]. We decided to examine if heart rate would enable monitoring the progression of K. pneumoniae pulmonary disease in comparison to BLI. The highest dose of meropenem (400 mg/kg/day) was associated with very early eradication of the 
bacteria where lung bioluminescence were below 3 standard deviation from background mean (Figure 16E). The increase in bacterial replication in the thoracic cavity monitored by bioluminescence coincided with decreased heart rate (Figure 16B - D and 16F - I). Interestingly, we observed that bioluminescence was able to detect the development of the respiratory disease by $15.71 \pm 3.672 \mathrm{hr}(p=0.0008)$ earlier than heart rate.

Collectively, the bioluminescent JSKP001 strain allowed for noninvasive monitoring of the therapeutic efficacy of meropenem against $K$. pneumoniae respiratory infection. Similar to previous studies, we demonstrated that the development of the pulmonary disease is associated with bradycardia [268]. However, our data suggest that BLI allow for earlier prediction of disease outcomes relative to heart rate. We propose that the bioluminescent $K$. pneumoniae strain together with our lung-specific disease model might serve as an improved model for preclinical testing of innovative antimicrobial agents against the respiratory disease caused by this important pathogen.

\subsubsection{Tracking the clearance of manC mutant using optical diagnostic imaging}

We decided to investigate whether optical diagnostic imaging will facilitate a better understanding of $K$. pneumoniae pathogenesis by testing if the clearance of a manC capsule mutant, known to be attenuated in murine intranasal disease model [92], could be monitored using BLI. The manC gene (also known as $c p s B$ ) encode for mannose-1-phosphate guanylyl transferase which is involved in the 
synthesis of GDP-D-mannose that gets converted into GDP-L-fucose, the precursor of fucose [269]. A manC mutant was generated in JSKP001 using allelic exchange mutagenesis creating the strain JSKP001 $\Delta$ manC. Albino C57BL/6J mice were infected with JSKP001 and JSKP001 $\Delta$ manC strains by IMIT using $10^{4.1}$ and $10^{8}$ bacterial inoculums, respectively. The $10^{8} \mathrm{CFU}$ represent the highest dose we instill using IMIT. Deleting the manC gene attenuated the ability of $K$. pneumoniae to cause the respiratory disease in our lung-specific disease model by $>3.9$ log relative to its parental strain (Figure $17 \mathrm{~A}$ ), in which animals infected with the wild type strain demonstrated $80 \%$ mortality while $0 \%$ mortality was observed in mice infected with the JSKP001 $\triangle$ manC strain. Thoracic cavity bioluminescence of mice infected with the capsule mutant was below the limit of detection (LOD) as early as $18 \mathrm{hr}$ post infection suggesting early clearance of the bacteria (Figure 17B). Bacterial burdens estimated from blood, BAL, lung, liver, spleen, and kidneys at 2 weeks post infection from mice inoculated with the manC mutant were less than bacterial numbers estimated for the same tissues collected from moribund animals infected by the parental strain (Figure 17C). For most tissues bacterial culture did not detect capsule mutant with the exception being the lung where there was low burden of $\sim 3.52$ logs at two weeks post infection.

We next decided to investigate whether diagnostic imaging can be used to noninvasively monitor clearance of an attenuated mutant. We looked at the capsule mutant which we know to be attenuated at $10^{8} \mathrm{CFU}$, and knowing that the bacteria is going to be undetectable by bioluminescence signal by $18 \mathrm{hr}$, we 
started imaging the animals at earlier time points and euthanized them at $6.5 \mathrm{hr}$. Mice were infected with $10^{8} \mathrm{CFU}$ of the JSKP001 $\operatorname{man} C$ strain and imaged at $0.5,3.5$, and $6.5 \mathrm{hr}$ post infection followed by euthanasia and lungs bacterial burdens estimation. Using thoracic cavity bioluminescence, we observed initial bacterial growth by $3.5 \mathrm{hr}$ post infection in half of the animals, which was followed by a subsequent drop in bacterial numbers in all mice lungs at $6.5 \mathrm{hr}$ suggesting initiation of clearance after $3.5 \mathrm{hr}$ of infection (Figure 18A). The average bacterial load in mice lungs estimated at $6.5 \mathrm{hr}$ post infection is $\sim 7.83 \pm 0.31$ log CFU which is $\sim 0.2 \log$ less than the inoculum ( $8 \mathrm{log}$ ) used to infect the animals again suggesting the initiation of clearance (Figure 18B). The amount of bioluminescence estimated from life animal at the $6.5 \mathrm{hr}$ correlated very well with lungs bacterial numbers enumerated following euthanasia (Figure 18C) demonstrated bioluminescence is a direct measurement of bacterial numbers similar to previous observations (Figure 13), and that removing bacterial CPS did not impact the ability to monitor bacterial viability in vivo using BLI. Together, these data demonstrate that the manC mutant is attenuated in our IMIT infection model in consistence with previous findings demonstrating the attenuation of a manC (or $c p s B$ ) mutant in mouse intranasal infection model [92]. Furthermore, our data suggest that the clearance of the $\Delta m a n C$ is likely initiated within few hours post infection $(3.5-6.5 \mathrm{hr})$ and the development of the optical diagnostic tools facilitate observing these interesting phenomena. 


\subsubsection{Monitoring intracellular growth of $K$. pneumoniae}

While often considered as an extracellular pathogen, we have recently demonstrated that $K$. pneumoniae is capable of replicating within cultured murine macrophages [99]. Therefore, we decided to investigate whether the intracellular proliferation of the bacteria can be monitored by bioluminescence. RAW264.7 murine macrophages were infected with JSKP001 and ATCC 43816 using MOI of 10 and bacterial proliferation was monitored at $3,4.5,6$, and $9 \mathrm{hr}$ post infection using light measurement followed by lysing the cells and estimating bacterial numbers. Interestingly, even though the JSKP001 behaved like the parental strain where both bacteria were viable and replicating within the cells, over the short period of observation, JSKP001 bioluminescence was dropping exponentially (Figure 19). To determine if the loss of bioluminescence upon bacterial internalization is due to an issue with the $r p / U$ promoter, we have generated bioluminescent strains with other promoters including $r p o D, o m p C$, EM7, and frr, and tested the ability of the bacteria to generate bioluminescence within host cells. J774A.1 murine macrophages were infected with MOI of 10 with the different strains, extracellular bacteria were killed using gentamicin treatment, and bioluminescence was monitored at 2, 3, 4, 5, 6, 9, and $12 \mathrm{hr}$ post infection for intracellular bacteria. Only the strains with the $r p o D$ and frr promoters were capable of generating a detectable bioluminescence at early time points followed by a time-dependent loss of bioluminescence similar to the JSKP001 strain (Figure 20). These outcomes suggest that the loss of bioluminescence for internalized $K$. pneumoniae is not due to a promoter issue but rather the bacteria 
might be altering its metabolism after becoming intracellular as a way of adapting to that environment.

Since we have shown that $K$. pneumoniae can survive in cultured macrophages, we next decided to determine if we could isolate intracellular bacteria during the respiratory disease. Mice were infected with $10^{5.1} \mathrm{CFU}$ using the JSKP001 strain and the amount of intracellular bacteria were estimated $43 \mathrm{hr}$ post infection from lung homogenates. The estimation of the internalized bacteria was performed using two approaches: gentamicin protection assay and a filtration technique. Interestingly, a small percentage $(<1 \%)$ of $K$. pneumoniae was internalized by host cells within the lungs and similar numbers were obtained from gentamicin protection and the filtration assays (Figure 21). Together, in addition to the previous observation about the capability of $K$. pneumoniae to proliferate within cultured macrophages, we demonstrated that a subpopulation of the bacteria become intracellular within mice lungs and it would be interesting to investigate the role of these internalized microbes in disease.

\subsection{DISCUSSION}

This work demonstrates that the bioluminescent $K$. pneumoniae strain we engineered can be utilized to monitor therapeutic efficacy in a surrogate animal infection model. BLI will minimize the number of animals needed in time course studies while at the same time allow for real-time monitoring of bacterial burden in live host. Using the JSKP001 strain, we were able to monitor bacterial dissemination to anatomical sites other than the initial site of infection (Figure 
$12 \mathrm{~A})$, allowing the performance of studies directed toward characterizing secondary infections initiated following the pulmonary disease. The correlation observed between bioluminescence and bacterial numbers in culture media and in vivo makes the bioluminescent $K$. pneumoniae strain suitable for the performance of multiple studies including high throughput work while reducing the time, effort, and cost required for estimating viable microbes and eliminating the need for the invasive manipulation of host and bacteria. Interestingly, the high colonization of blood, liver, and kidneys is consistent with the ability of $K$. pneumoniae in causing septicemia, pyogenic liver abscess, and UTI in clinics [270-272].

Bioluminescence enabled monitoring K. pneumoniae viability upon treating the bacteria with meropenem where both $\mathrm{OD}_{600}$ and light measurement gave similar outcomes. In addition, we provided an evidence that bioluminescence can be used as an alternative approach to estimate antimicrobial agents $\mathrm{EC}_{50}$ to the traditionally used $\mathrm{OD}_{600}$ measurement. Optical diagnostic imaging allowed for earlier prediction of disease outcomes, in which higher bacterial burdens in mice lungs correlated with quicker development of moribund disease (Figure 12C).

In consistence with previous findings, we have demonstrated that deleting manC gene attenuated the ability of $K$. pneumoniae to cause the respiratory disease in our lung-specific disease model [92]. These outcomes is consistent we our previous finding demonstrating that removing K. pneumoniae capsule made the bacteria more susceptible to uptake by macrophages [99]. Thus, 
resisting uptake by host immune cells play an important role in bacterial virulence in vivo during the respiratory disease but it is probably not the only factor. More importantly, we have demonstrated in current work by bioluminescence imaging (BLI) that the clearance of the capsule mutants is an early even that gets initiated within hours following infecting the animals.

Using the bioluminescent strain, we identified the interesting phenomena that K. pneumoniae turn off bioluminescence when become intracellular suggesting that the bacteria might alter its metabolism to adapt to that environment. This intriguing observation led us to investigate how intracellular Klebsiella can get within host lungs and in consistence with the general notion about the extracellular property of $K$. pneumoniae we identified that $>0.2 \%$ of the bacteria are detectable as being internalized into host cells in vivo. To the best of our knowledge, this is the first demonstration that a subpopulation of $K$. pneumoniae becomes intracellular during the respiratory disease. This subpopulation may represent $K$. pneumoniae being successfully cleared by professional phagocytes and/or K. pneumoniae which may resist killing after internalization as we have observed in our cell culture models (Figure 6). These findings support the classification of $K$. pneumoniae as an extracellular pathogen, and future studies will be required to determine whether the intercellular $K$. pneumoniae contribute to disease.

Unlike other pathogens, capable of causing acute respiratory infection, animal infected with the same lethal dose of JSKP001 strain succumb to the infection at a broad MTTD range suggesting variable host susceptibility to $K$. 
pneumoniae pulmonary disease (Figure 10A). Even though we have an excellent and reproducible lung-specific disease model [187], we observed $\sim 1.3$ log differences in bacterial lung colonization by $18 \mathrm{hr}$ post infection (Figure 12B), which indicates a variation either in the bacteria as it try to evade killing by the host immune system or differences in host response to the invading pathogen. Future studies will need to be conducted to examine the contribution of host immune system in such phenomena. It is well known that neutrophil play an important role during $K$. pneumoniae respiratory disease [92, 102, 105-109, 113]. Thus, one way of testing if the variation observed in bacterial burdens at early time points is due differences in host immune response is by testing the recruitment of neutrophils at those time points by measuring blood cytokine levels involved in this process such as IL-8 and IL-17 [113, 273, 274].

In the current study, we decided to use albino C57BL/6J mice as initial studies demonstrated that this strain is more resistant than the previous experience with the exquisitely sensitive BALB/c model to direct lung installation of ATCC 43816 [99, 275]. Furthermore, the main reason behind using the albino C57BL/6J in our studies was due to the availability of the genetic tools in that background for future investigation. The $\mathrm{LD}_{50}$ estimated for the JSKP001 strain in albino C57BL/6J mice is $\sim 13.4$ fold higher than the LD 50 previously estimated for the parental strain ATCC 43816 in BALB/c further confirming the higher susceptibility of the BALB/c mice to the $K$. pneumoniae respiratory infection. In addition, blood, lung, liver, and spleen bacterial numbers for ATCC 43816 in moribund albino C57BL/6J mice were $\sim 0.3-1.6$ logs higher than the bacterial 
loads observed previously in BALB/c mice for the same tissues [99]. These differences illustrate a significant variation in the susceptibility of the two mice strains to the pneumonic disease [99]. The differences in the susceptibility of the albino $\mathrm{C} 57 \mathrm{BL} / 6 \mathrm{~J}$ and $\mathrm{BALB} / \mathrm{c}$ mice to $K$. pneumoniae respiratory disease might be attributed to the genotypic differences of these mice strains $[276,277]$. It is well known that C57BL/6 and BALB/c mice have different immune responses during health and disease [276-278] with C57BL/6 being mainly Th1 proinflammatory type response and BALB/c being more of a Th2 type responder with more humoral protection [279-286]. For acute disease model it would be predicted that a mouse line favoring a Th1 response will more readily compact infection.

In conclusion, we generated a bioluminescent version of $K$. pneumoniae ATCC 43816 which is frequently used strain in biomedical research. The bacterial fitness was not altered both in culture media and within the host. We demonstrated that bacterial viability upon antimicrobial treatment can be monitored both in vitro and in vivo model systems using light measurement. Bioluminescence can serve as an alternative way to traditionally used approaches for calculating antimicrobial agents $\mathrm{EC}_{50}$. Furthermore, the bioluminescent bacteria allowed for direct measurement of meropenem therapeutic efficacy against $K$. pneumoniae pulmonary disease with earlier prediction of disease outcomes. 

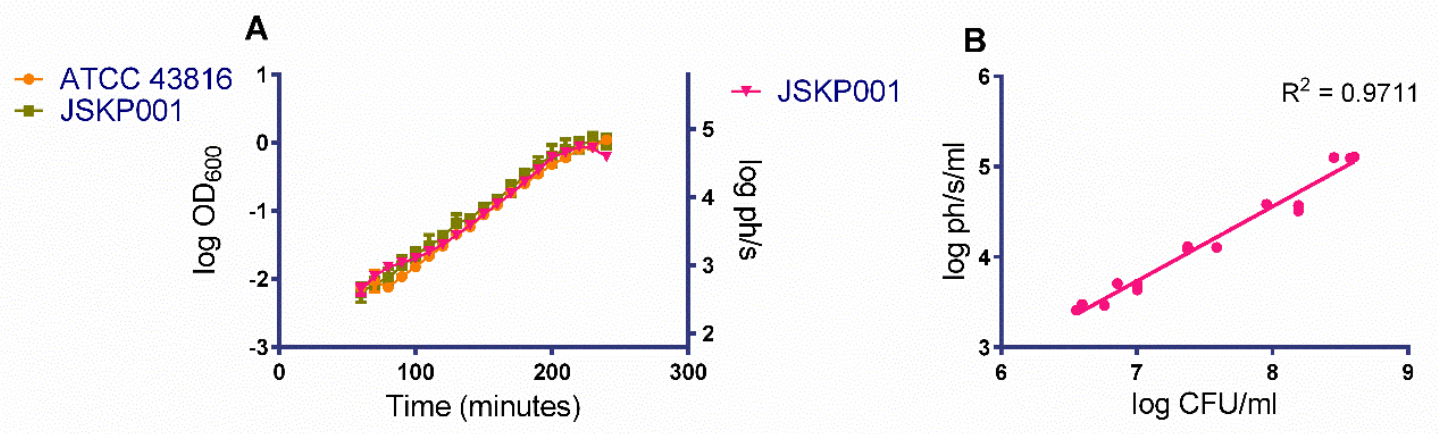

Figure 9. Growth curves of JSKP001 and ATCC 43816 strains. Overnight cultures of ATCC 43816 and JSKP001 were diluted 1000 fold in LB broth media and bacterial growth were monitored using Synergy ${ }^{\mathrm{TM}} \mathrm{H} 1$ plate reader for $4 \mathrm{hr}$ at $37^{\circ} \mathrm{C}$ where $\mathrm{OD}_{600}$ and bioluminescence reads were taken every $10 \mathrm{~min}(\mathrm{~A}) .(\mathrm{B})$ One thousand fold diluted overnight bacterial culture of JSKP001 strain were grown at $37^{\circ} \mathrm{C}$ for $3 \mathrm{hr}$ where $100 \mu \mathrm{l}$ aliquots were taken every $30 \mathrm{~min}$ for bioluminescence and bacterial numbers enumeration by serial dilution. 

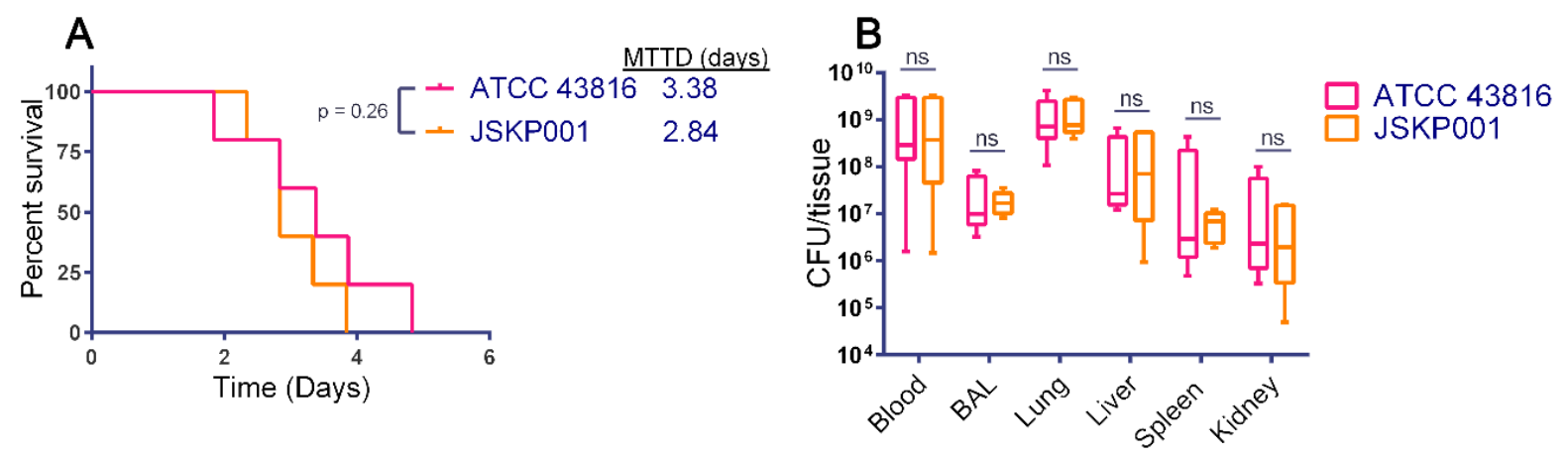

Figure 10. Survival analysis and bacterial systemic dissemination. Albino

C57BL/6J (8-10 weeks) were intratracheally infected with either ATCC 43816 (104.95 CFU), or JSKP001 (104.91 CFU), and the survival curves from both animal groups $(n=5)$ were compared $(A)$. Survival analysis is indicated on the figure. (B) Bacterial burdens of lungs, liver, spleen, kidneys, BAL, and blood estimated from moribund animals by plate count. (MTTD, median time to death; ns, not significant) 


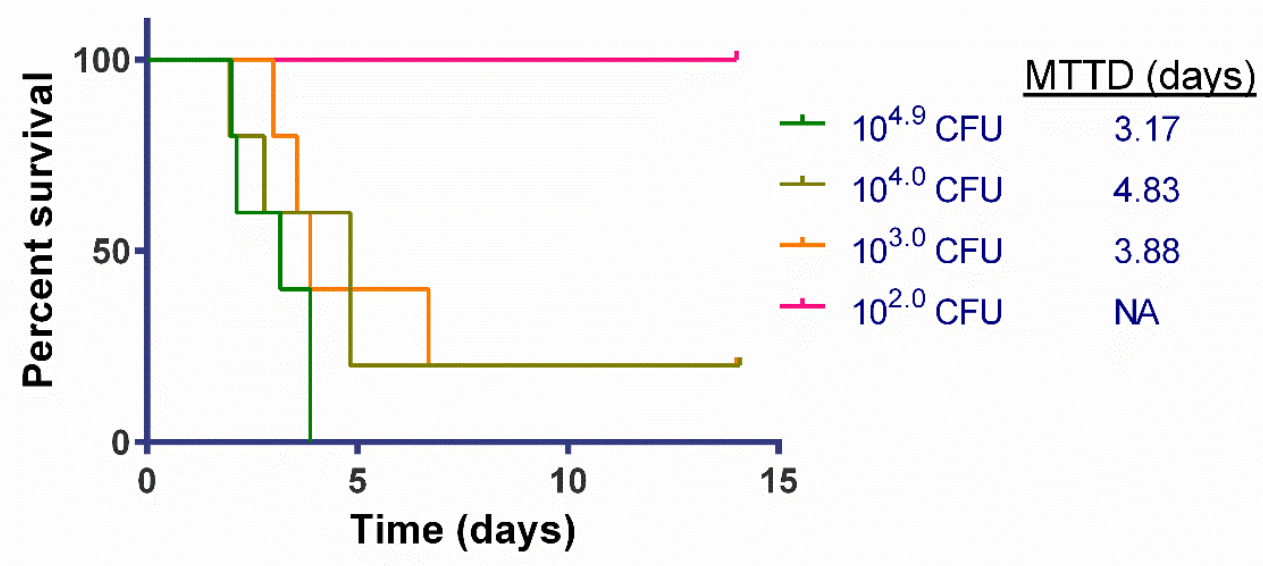

Figure 11. JSKP001 LD50 determination in albino C57BL/6J mice. Mice $(n=$ 5) were infected with $10^{2}-10^{4.9}$ CFU of JSKP001 using IMIT, monitored for the development of the pneumonic disease over two week preiod, and eutanized when moribund. Animals median time to death (MTTD) is indicated on the figure. (NA: not applicable). 

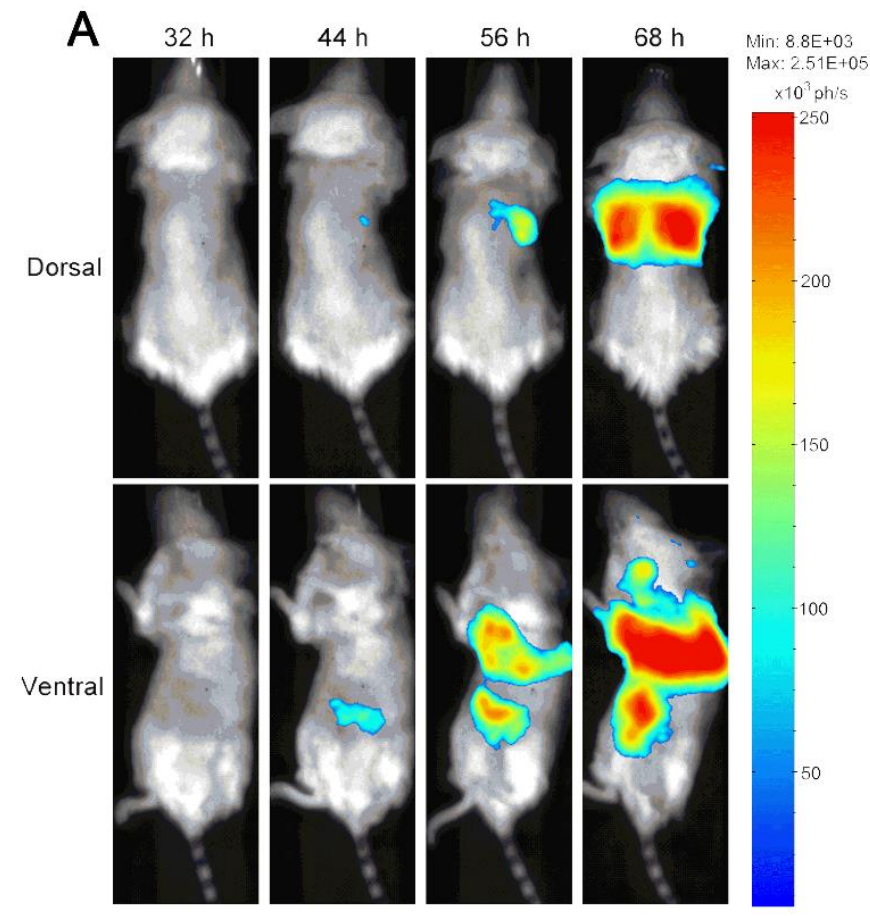

B

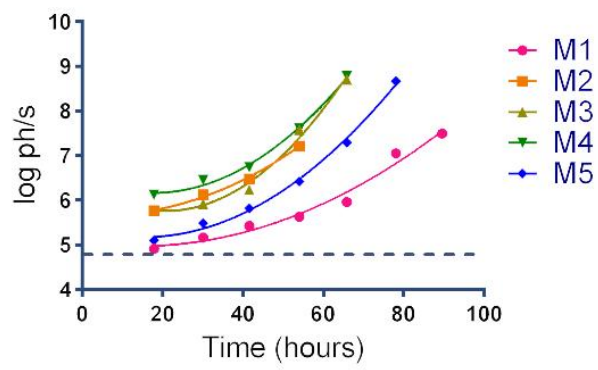

C

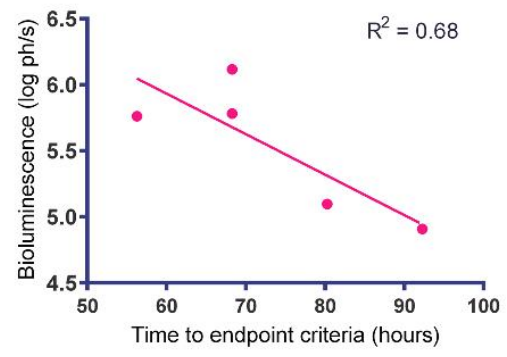

Figure 12. In vivo tracking of bacterial proliferation. Albino C57BL/6J mice (n = 5) infected with JSKP001 (104.9 CFU) using IMIT and monitoed for the development of the respiratory disease using PhotonIMAGER Optima. (A) Representative mouse (M4) imaged for 1 min on dorsal (top panel) and ventral (bottom panel) positions. (B) Bioluminescence imaging of mice thoracic cavity (dorsal position) monitored twice daily. The dotted lines represent two standard deviation limit of detection (LOD) estimated from uninfected animals. (C) Correlation between bacterial burdens in mice thoracic cavity by BLI and time to euthanasia. 

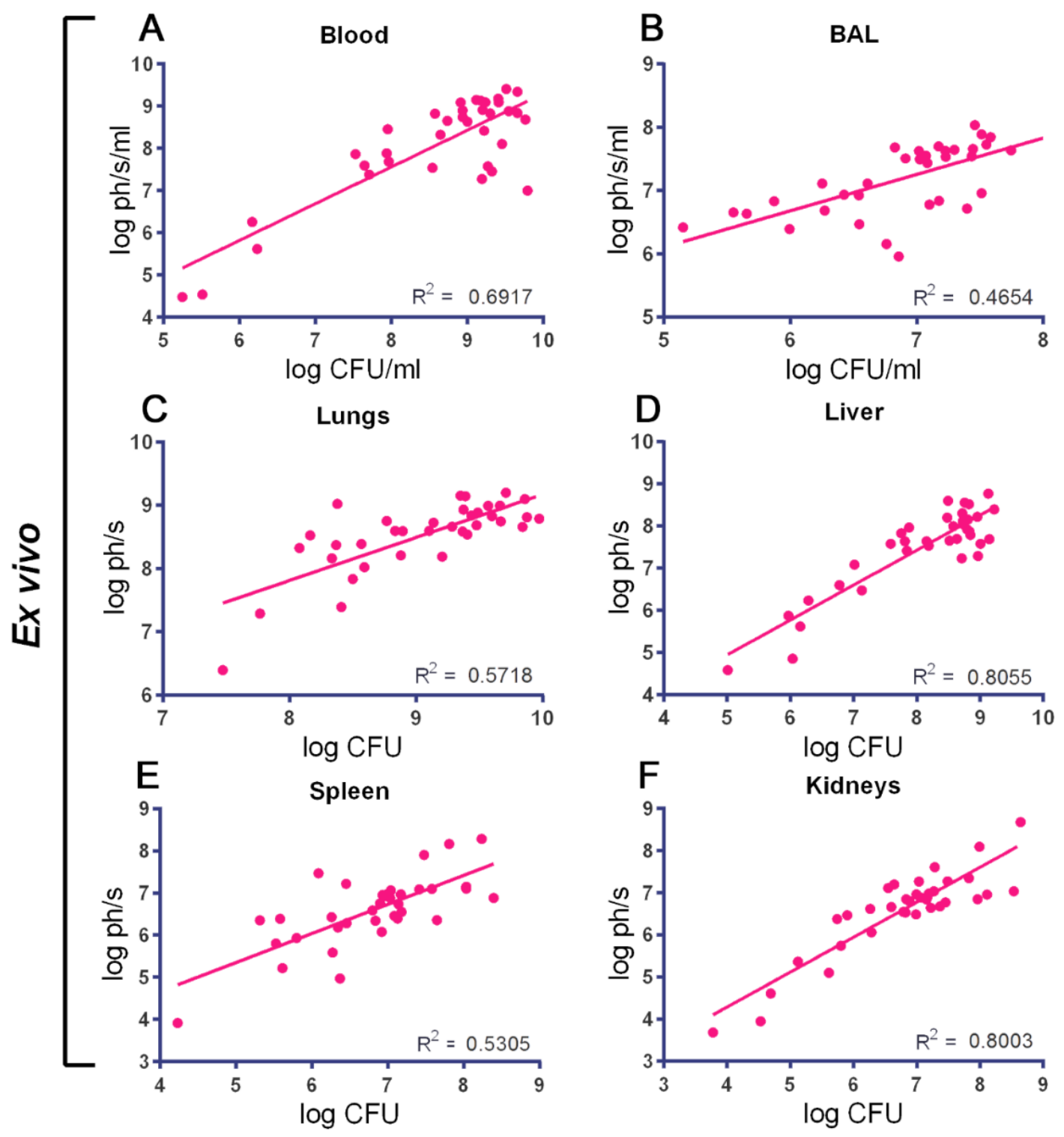

G

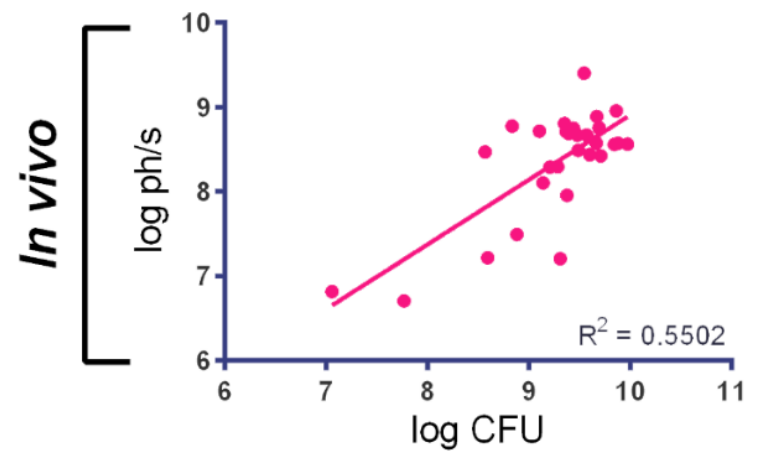


Figure 13. Correlation of bioluminescence and bacterial burdens in host tissues. Lung, liver, spleen, kidneys, BAL, and blood samples collected from moribund animals $(n=36)$ introduced with $10^{3}-10^{5.2}$ bacteria using direct lung installation. $(A-F)$ Ex vivo bioluminescence was measured using PhotonIMAGER Optima, and bacteria were enumerated from the processed samples. (G) Correlation between in life lungs bioluminescence before euthanasia and bacterial burdens from tissue homogenates. Ex vivo and in vivo correlations represented by the $\mathrm{R}^{2}$ values are indicated on the figure. In vivo bioluminescence from mice thoracic cavity were estimated before collecting the BAL while the ex vivo bioluminescence from the lung tissues were estimated following the BAL wash. 

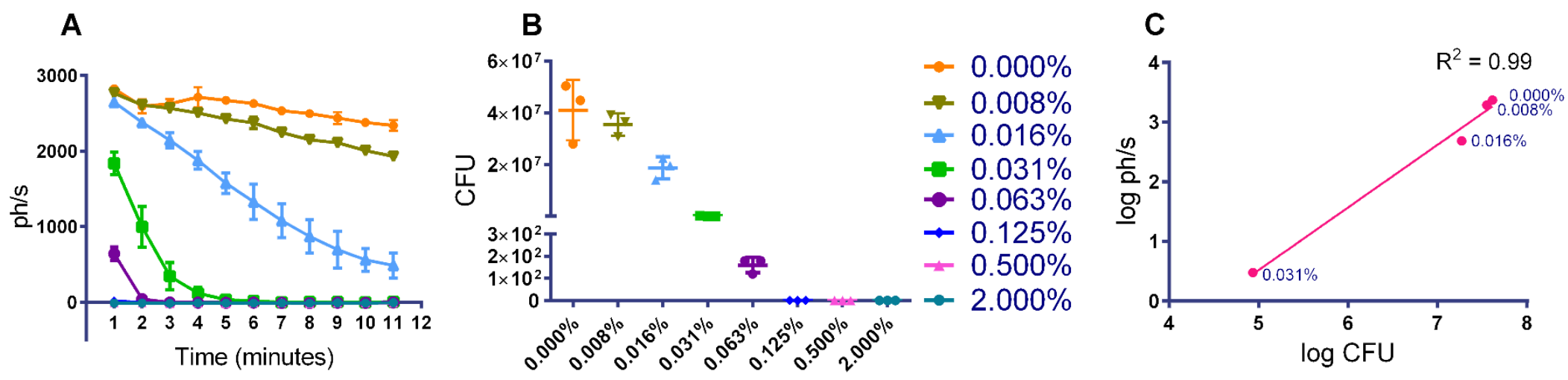

Figure 14. Tracking bacterial viability upon MicroChem-Plus treatment using bioluminescence. JSKP001 viability upon treating the microbes with different concentrations of MicroChem-Plus disinfectant tracked by bioluminescence $(A)$ and by bacterial numbers (B) estimated at 11 min post treatment. (C) Correlation between bacterial numbers and bioluminescence at $11 \mathrm{~min}$ from wells with viable bacteria. 

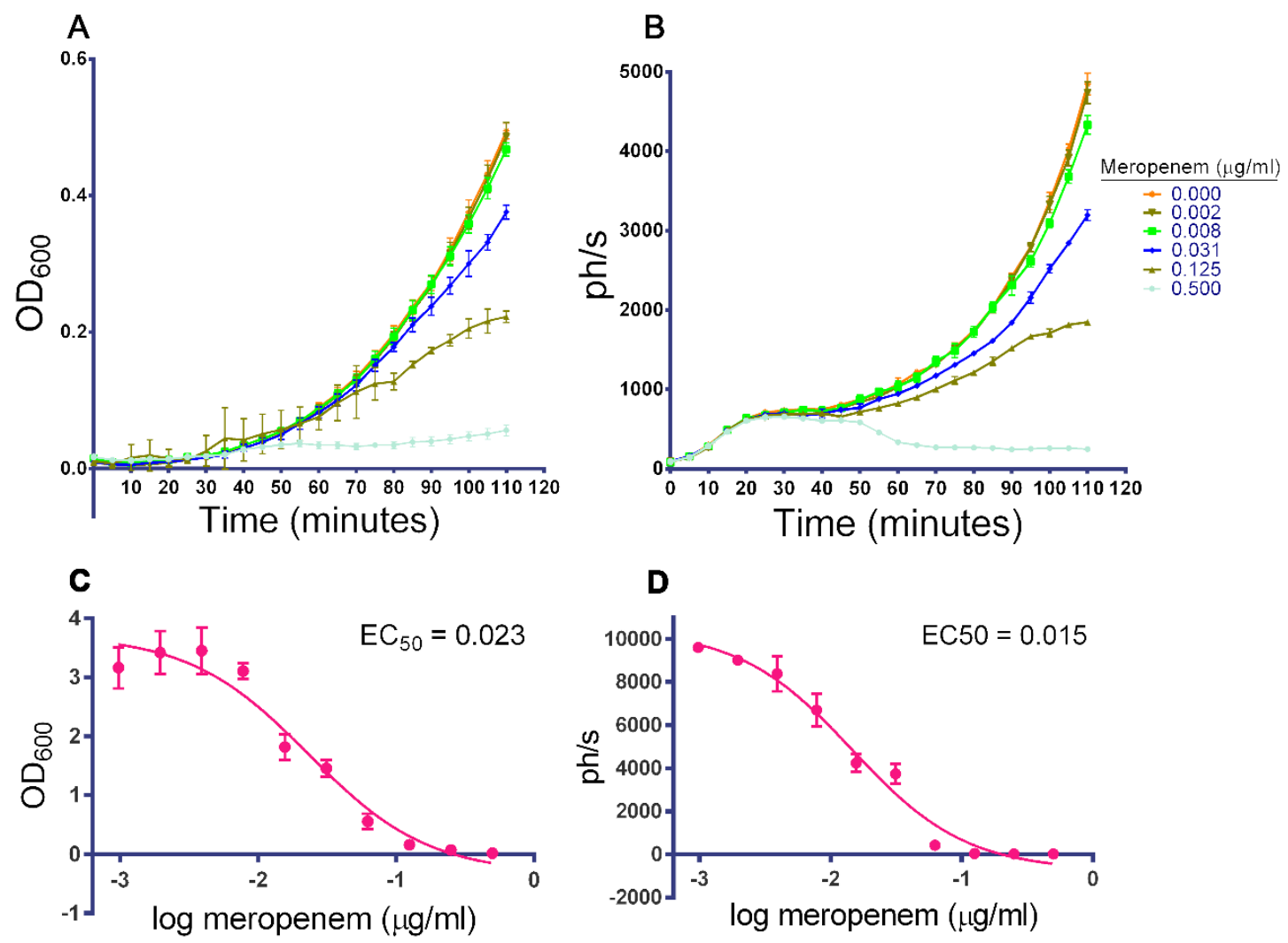

Figure 15. Monitoring the impact of meropenem treatment on bacterial growth using bioluminescence. Exponentially growing JSKP001 bacteria were diluted ( $\left.10^{5.9} \mathrm{CFU}\right)$, treated with serially diluted meropenem, and bacterial growth was monitored by $\mathrm{OD}_{600}(\mathrm{~A})$ and bioluminescence $(\mathrm{B})$ at $5 \mathrm{~m}$ intervals. Meropenem $\mathrm{EC}_{50}$ estimated by $\mathrm{OD}_{600}(\mathrm{C})$ and bioluminescence (D) at $8 \mathrm{hr}$ posttreatment. 
A
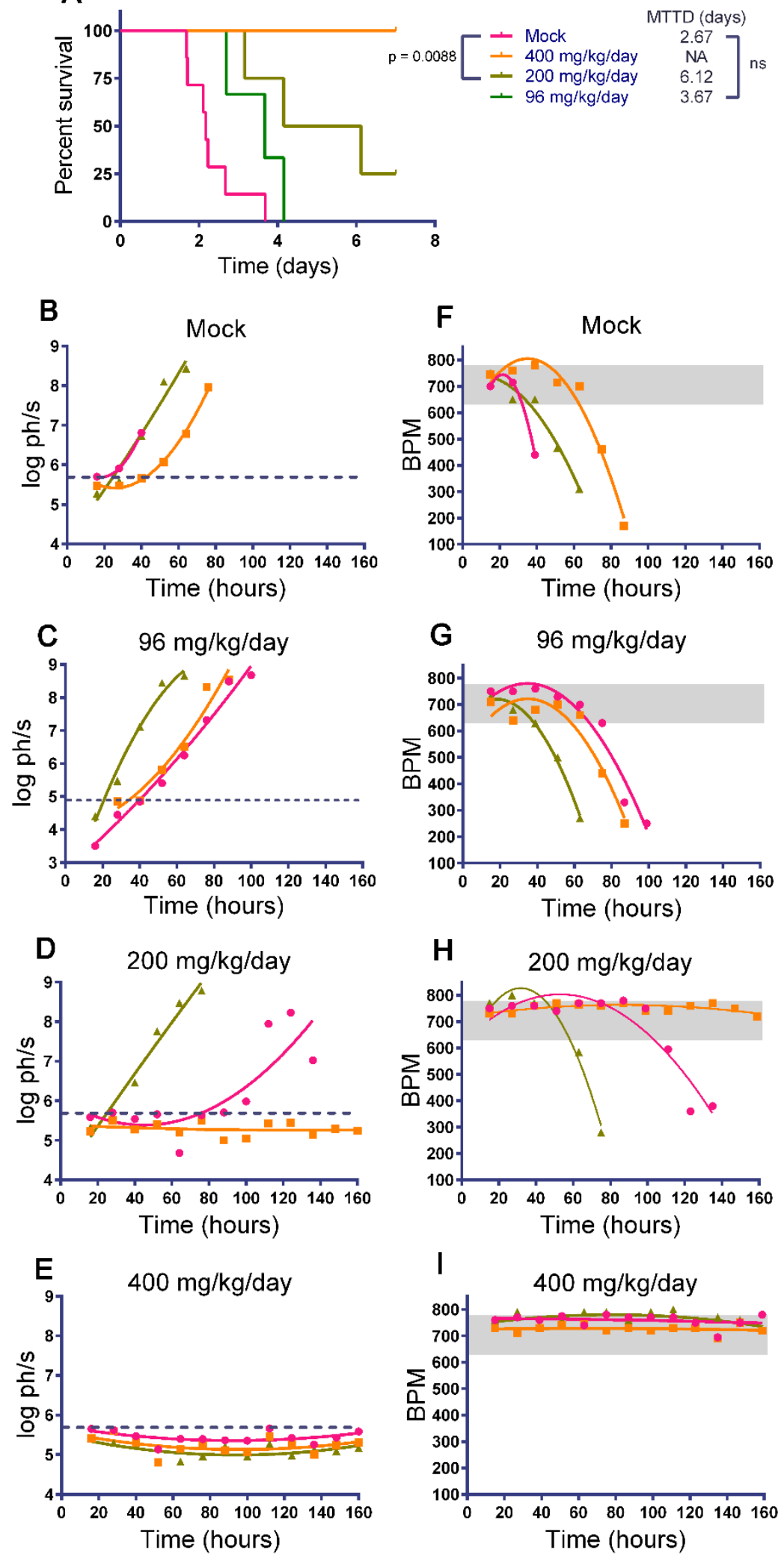

$\mathrm{M} 1 \div \mathrm{M} 2 \div \mathrm{M} 3$ 
Figure 16. In vivo monitoring of meropenem efficacy against $K$. pneumoniae respiratory infection in mice. Lethally infected $\left(10^{4.9} \mathrm{CFU}\right)$ albino C57BL/6J mice 8-10 weeks $(n=3)$ were treated with subcutaneous (s.c.) injection q12h of 400, 200, $96 \mathrm{mg} / \mathrm{kg} /$ day meropenem, or PBS vehicle. (A) Survival curves of mice receiving the different therapeutic treatments. Survival analysis and median time to death (MTTD) is indicated on the figure. (B - E) Animals monitored for the development of the respiratory disease twice a day by tracking bioluminescence emitted from the thoracic cavity (dorsal image). (F-I) Mice heart rates estimated twice daily using MouseOx system. The dotted lines and the gray shades represent three standard deviation from background mean. (NA, not applicable; ns, not significant). 

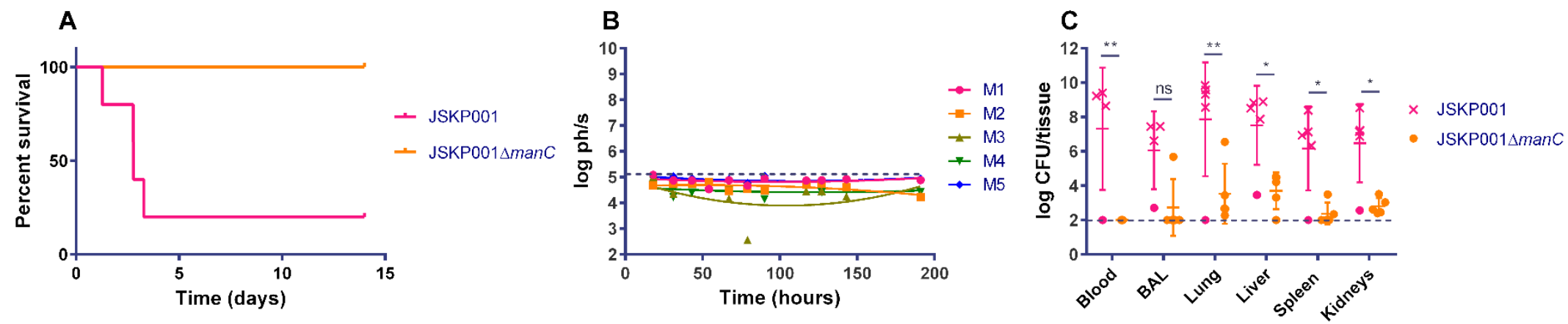

Figure 17. Noninvasive monitoring of JSKP0014manC fitness in mice. Albino C57BL/6J mice $(n=5)$ infected with JSKP001 (104.1 CFU) or JSKP001 $\triangle$ manC $\left(10^{8} \mathrm{CFU}\right)$ using IMIT and monitored for development of the respiratory disease for two weeks. (A) Survival curves of animals infected with the manC mutant and wild type parental strain. (B) Bacterial burdens of capsule mutant in mice thoracic cavity monitored by optical diagnostic imaging. (C) Bacterial burdens in tissues collected following euthanasia upon moribund disease or 14 days post infection. The closed circles $(\bullet)$ represent animals that survived the respiratory disease. Dotted lines represent two log limit of detection. (ns, not significant; $\left.{ }^{*}, p<0.05 ;{ }^{* *}, p<0.001\right)$. 

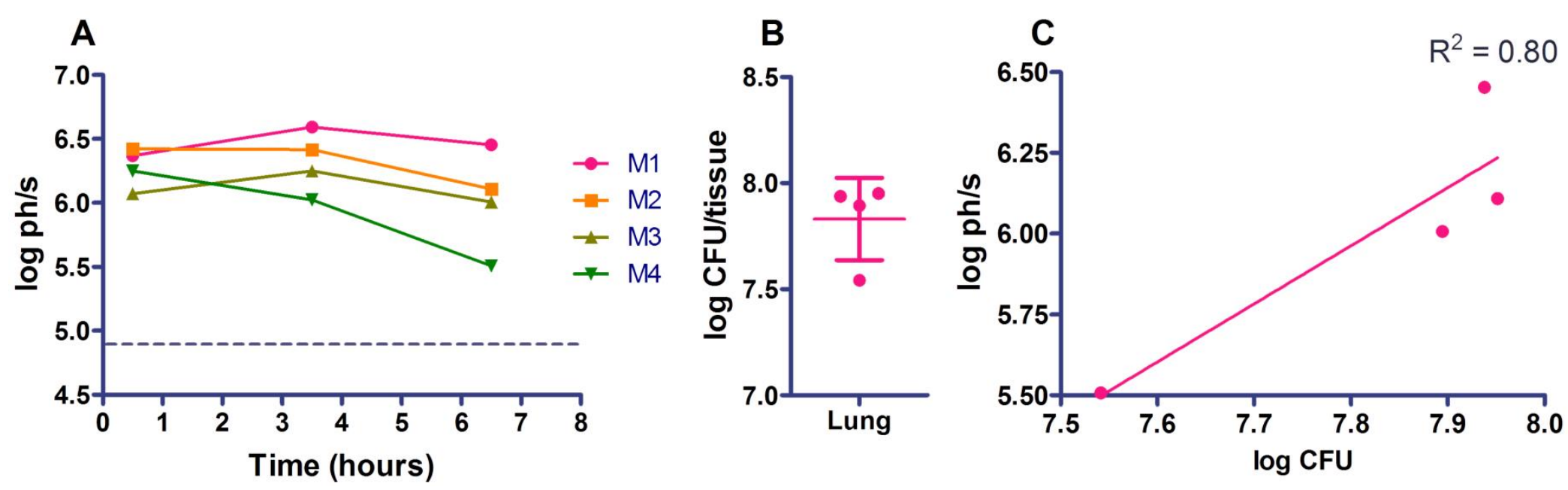

Figure 18. Tracking the clearance of the capsule mutant using bioluminescence imaging. Albino female C57BL/6J mice $(n=4)$ were infected with $10^{8}$ CFU with JSKP001 $\Delta$ manC using IMIT. (A) Bacterial proliferation within animals' lungs tracked using BIOSPACE LAB PhotonIMAGER Optima system at $0.5,3.5$, and $6.5 \mathrm{hr}$ post infection. (B) Bacterial burdens enumerated from mice lungs $6.5 \mathrm{hr}$ post infection. (C) Correlation between thoracic cavity bioluminescence in life and bacterial burden in mice lungs following euthanasia. 
$\rightarrow$ JSKP001

- ATCC 43816

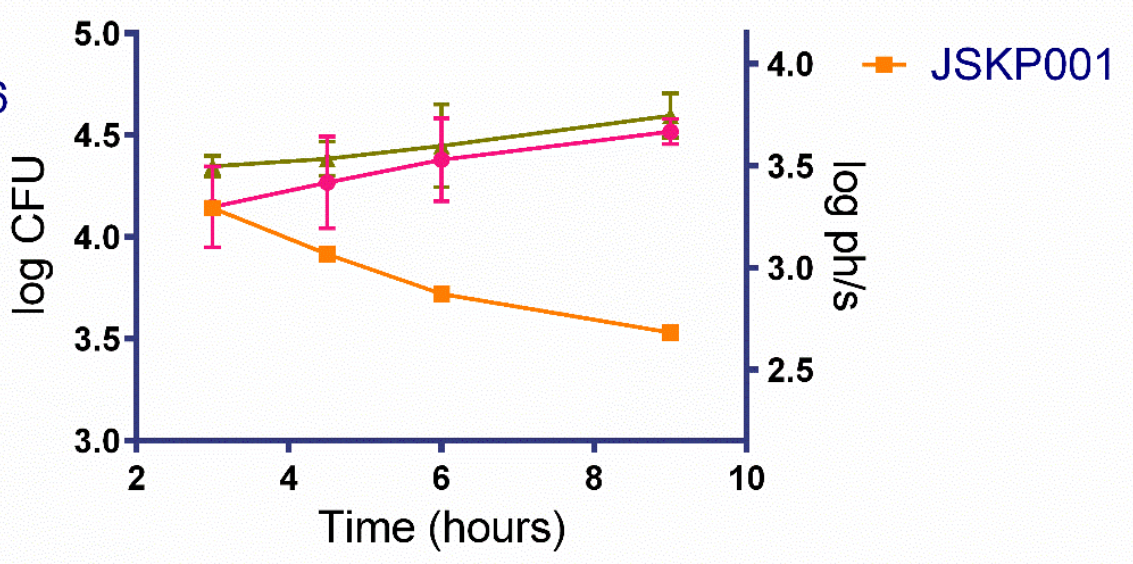

Figure 19. Monitoring bacterial replication within cultured macrophages.

Gentamicin protection assay was performed where RAW264.7 murine cells were infected with JSKP001 or ATCC 43816 using MOI of 10 and bacterial numbers were estimated at $3,4.5,6$, and $9 \mathrm{hr}$ post infection. Bioluminescence was monitored for the JSKP001 strain at the same time points using Synergy ${ }^{\mathrm{TM}} \mathrm{HT}$ plate reader. 


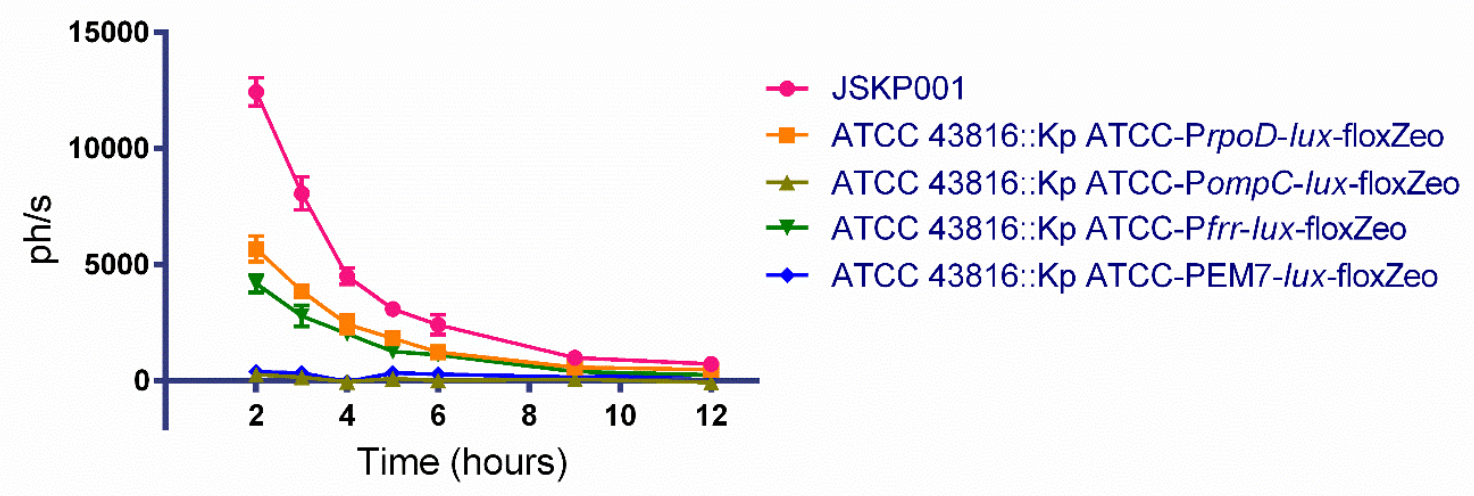

Figure 20. Tracking $K$. pneumoniae proliferation within cultured macrophages using different promoter systems. J774A.1 cells were infected with $\mathrm{MOI}$ of 10 using the strains indicated in the figure, extracellular bacteria were killed using gentamicin treatment at $1 \mathrm{hr}$, and intracellular bacteria were monitored at 2, 3, 4, 5, 6, 9, and $12 \mathrm{hr}$ post infection by measuring bioluminescence using Bio-tek Synergy ${ }^{\mathrm{TM}}$ HT plate reader. 


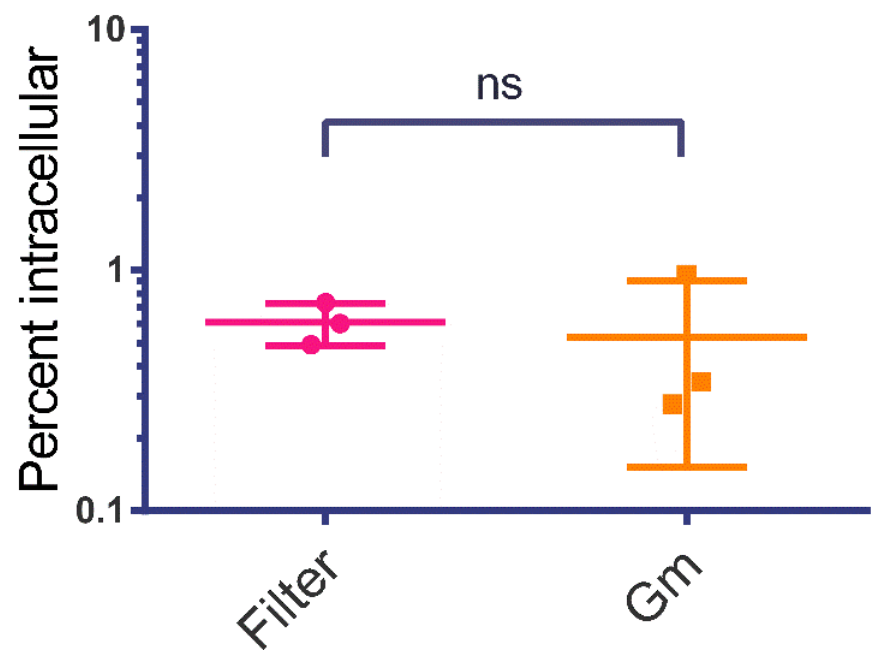

Figure 21. Estimating bacterial internalization into host cells within mice lung. Albino C57BL/6J mice were intratracheally infected with JSKP001 (10 5.1 CFU). Animals were euthanized $43 \mathrm{hr}$ post infection, lungs were collected, dissociated into single cell suspension using collagenase treatment, and total bacterial loads were estimated following treating the tissue homogenates with Triton X-100. Intracellular bacteria were estimated by running $10^{6}$ cells through 5 micron filter to wash off extracellular microbes followed by lysing the cells using Triton X-100 for intracellular bacterial estimation. Internalized bacteria were also estimated from $10^{6}$ cells using gentamicin protection assay. Percent intracellular represent the percentage of the internalized $K$. pneumoniae to the total bacterial numbers in the mice lungs. (ns: not significant). 


\section{CHAPTER 5}

\section{DISCUSSION AND FUTURE DIRECTIONS}

Collectively, we have provided the first draft of the K. pneumoniae ATCC 43816 sequence and compared the genome of that strain to two previously fully sequenced K. pneumoniae strains, and identified shared known and potential virulence determinants between ATCC 43816 and NTUH-K2044, disease causing strains, that are absent from the avirulent MGH 78578 strain. We studied the uptake of the three K. pneumoniae strains by cultured macrophages, investigated the involvement of the capsular polysaccharide (CPS) in this process, and provided the first evidence that $K$. pneumoniae strains are capable of intracellular proliferation. We established a unique pulmonary infection model where we can specifically deliver the microbes into mice lungs allowing us to study colonization of the lower respiratory tract (LRT). Using that model system we demonstrated that both ATCC 43816, NTUH-K2044 were highly virulent to BALB/c mice while MGH 78578 was relatively avirulent requiring a very high dose in order to cause the disease. We used the recently constructed bioluminescent JSKP001 strain to conduct multiple investigations including noninvasive monitoring of bacterial proliferation in broth media and in vivo, tracking of microbial viability upon bactericidal agents' treatment, estimating 
antimicrobial drugs $\mathrm{EC}_{50}$, monitoring the efficacy of therapeutic in vivo with earlier prediction of disease outcomes, and direct monitoring of the fitness of capsule mutant.

\subsection{Identifying virulence factors required by $K$. pneumoniae to establish the respiratory disease using Tn-seq}

Even though $K$. pneumoniae is an important pathogen capable of causing acute diseases in human, only few virulence determinants were identified to be required for the respiratory disease caused by these bacteria. Conversely, pathogens that cause similar infection such as Streptococcus pneumoniae, Staphylococcus aureus, Legionella pneumophila, Haemophilus influenza, and Yersinia pestis encode for an arsenal of virulence determinants that play a role in the disease induction [287-293]. In the case of Legionella pneumophila, it is thought is possesses over 300 proteins secreted by a type 4 secretion system to facilitate its intracellular lifestyle [287]. Therefore, we propose that there are other unidentified virulence factors required by $K$. pneumoniae to cause pulmonary disease within a host and the identification of these factors would help the discovery of new therapeutic targets for this critical pathogen.

Tn-seq is one of the most recent methods utilized for the identification of virulence factors required by a microbial pathogen [294-296]. It combines transposon mutagenesis together with massive parallel sequencing (MPS) to identify fitness factors required by a microorganism to survive under a set of growth conditions or selective pressures. We decided to employ Tn-seq for the 
purpose of identifying virulence factors that contribute into K. pneumoniae respiratory disease using the models developed by our group. A Tn-seq library was generated in the bioluminescent JSKP001 strain to enable monitoring the specific inoculation and proliferation of the mutants in host lungs. A library of 20,000 transposon mutants was generated representing approximately four-fold coverage of the K. pneumoniae genome size ( 5.4 MB).

Five mice were infected with $10^{4.8} \mathrm{CFU}$ by IMIT and monitored for the development of the respiratory disease by optical diagnostic imaging and euthanized upon reaching thoracic cavity bioluminescence of $10^{8} \mathrm{ph} / \mathrm{s}$ (Figure 22B), representing late stage disease. Median time to endpoint criteria was estimated to be 1.8 days (Figure 22A), which is consistent with previous data with the exception that we did not allow the animals to reach bradycardia moribund endpoint previously established. Bacterial burdens in mice lungs were $\sim 10^{9} \mathrm{CFU}$ (Figure 22C), also consistent with previous outcomes (Figure 10B). The chromosomal DNA was extracted from the inoculum (input pool) and from the transposon libraries collected from lungs homogenates (output pool) to be used in subsequent analysis. This is an ongoing that is being conducted in collaboration with Dr. Deborah Yoder-Himes. 

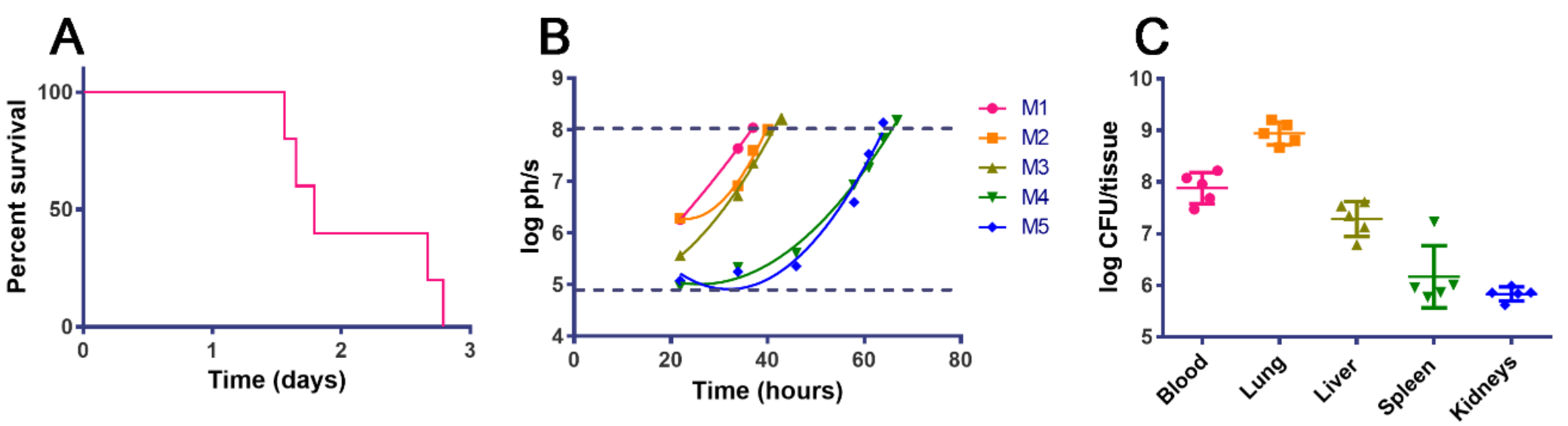

Figure 22. Noninvasive monitoring of JSKP001 Tn-seq library in lung-specific disease model. Albino

C57BL/6J mice $(n=5)$ infected with $10^{4.8} \mathrm{CFU}$ of JSKP001 transposon library using IMIT and bacterial colonization of host lungs were monitored using BIOSPACE LAB PhotonIMAGER Optima system. (A) Survival curve with estimated median time to endpoint criteria of 1.8 days. (B) Animals monitored for lung colonization by the transposon library using bioluminescence imaging and euthanized upon meeting in vivo lung bioluminescence of $10^{8} \mathrm{ph} / \mathrm{s}$. Upper dotted line represent the threshold for euthanasia and the lower one indicate 2 standard deviation limit of detection (LOD) estimated from uninfected animals. (C) Bacterial burdens for the indicated tissues collected following mice euthanasia. 


\subsection{The role of T6SS for K. pneumoniae in disease}

As the Tn-seq study is an ongoing work, we decided to investigate the role of other virulence systems that might play a role during K. pneumoniae respiratory disease. Type six secretion systems (T6SS) are among a recently identified secretion systems of Gram-negative bacteria that is functionally and evolutionary uniquely similar to T4 bacteriophage injectisome [297]. T6SSs play a role in virulence in multiple important pathogens as well as in bacterial competition [296, 298-302]. K. pneumoniae strains encode for $2-3$ clusters of T6SSs [303] and for ATCC 43816 K. pneumoniae strain it encodes two clusters of those secretion systems $[99,304]$. Previous studies have suggested a potential role for T6SS cluster 1 in K. pneumoniae intranasal murine model of respiratory disease, in which a mutant was less efficient in dissemination to spleen [92]. That mutant was identified in signature tagged mutagenesis but it was not further characterized. Due to the importance for T6SS for the virulence of other pathogens, we predicted that it might play a role in K. pneumoniae respiratory disease. Having developed the capability to manipulate $K$. pneumoniae genome, we decided to create T6SS mutants and investigate their importance for the pulmonary disease using our lung-specific disease model.

T6SS mutants were created through the deletion of the vgrG genes which are required for a functional secretion system [297]. Single vgrG knockouts were made in each T6SS cluster of the JSKP001 strain as well as in both clusters

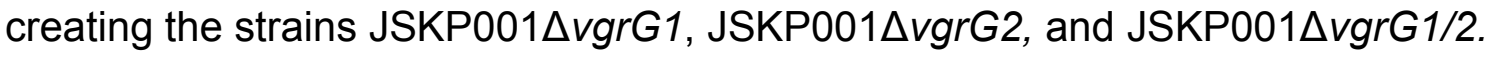
The fitness of the different $v g r G$ mutants were compared to their parental strain 
in albino C57BL/6J mice where the different strains were inoculated into animals' lungs with $10^{4.8}-10^{4.9} \mathrm{CFU}$ using IMIT. The vgrG mutants were as virulent as their parental strain in the single strain infection study performed with a MTTD ranging from $2.4-3.8$ days (Figure $23 \mathrm{~A}$ ). In addition, bacterial loads in blood, BAL, lungs, liver, kidneys, and spleen were similar between animals infected with the $\operatorname{vgrG}$ mutants and the wild type strain (Figure 23B). 

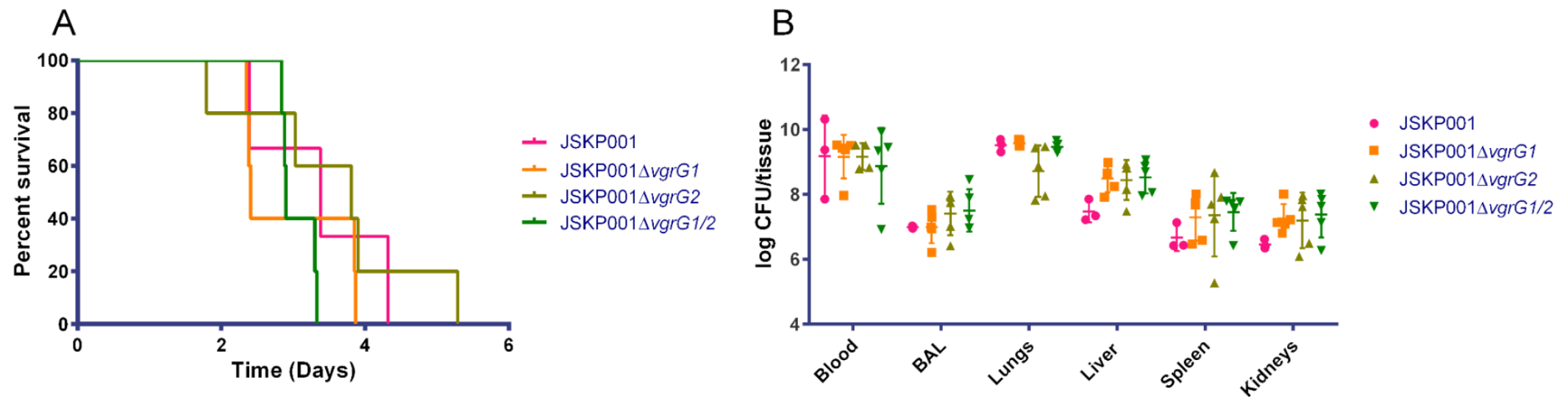

Figure 23. In vivo fitness of $K$. pneumoniae T6SS mutants. Albino C57BL/6J mice $(n=5)$ infected with $10^{4.8}$ $10^{4.9}$ bacteria of the $\operatorname{vgrG}$ mutants indicated the figure using IMIT. (A) Survival curves of the mice inoculated with the different $\operatorname{vgrG}$ mutants in comparison to the parental JSKP001 strain. Median time to death (MTTD) is indicated on the figure. (B) Bacterial burdens in tissues collected from moribund mice following euthanasia. 
Previous work demonstrated that competition studies is a higher resolution method for identifying subtle phenotypes that were indistinguishable by single strain infection [296], and thus we decided to employ this approach for testing the potential contribution of T6SS in K. pneumoniae respiratory disease. Each of the vgrG bioluminescent mutants was inoculated into albino C57BL/6J mice lungs in 1:1 ratio with the non-luminescent ATCC 43816 strain $\left(10^{4.9}-10^{5.4} \mathrm{CFU}\right)$. The animals were monitored for the development of the respiratory disease and euthanized upon meeting a heart rate of $500 \mathrm{bpm}$ or below. Following euthanasia, blood, lungs, liver, and spleen were collected and processed for bacterial enumeration. The luminescent bacteria were distinguished from the non-luminescent wild type strain using optical diagnostic tools allowing us to calculate output ratios of bacteria on LB plates and estimate in vivo bacterial burdens of the JSKP001 strains.

Interestingly, most of the animals infected with the vgrG1 knockout strain had reduced in vivo colonization of the lungs (Figure 24A), distinguished from the wild type ATCC 43816 strain by luminescence, and an alleviated competition indexes in comparison to ATCC 43816 in all tested tissues (Figure 24B. To confirm that the potential reduction of vgrG1 mutant fitness was not due to early euthanasia of the animals infected with that mutant we estimated the total bacterial burdens in the collected tissues. We found no pronounced difference in total bacterial numbers from all collected tissues suggesting that all animals were euthanized with the same level of disease (Figure 24C). Conversely, we observed no reduced fitness of the $v g r G$ double knockout strain which was 
expected to at least demonstrate similar phenotype to the vgrG1 mutant. These outcomes suggest that T6SS cluster 1 might be required for $K$. pneumoniae respiratory disease but due to the inconsistent observations more future investigations will be conducted to confirm such hypothesis. 

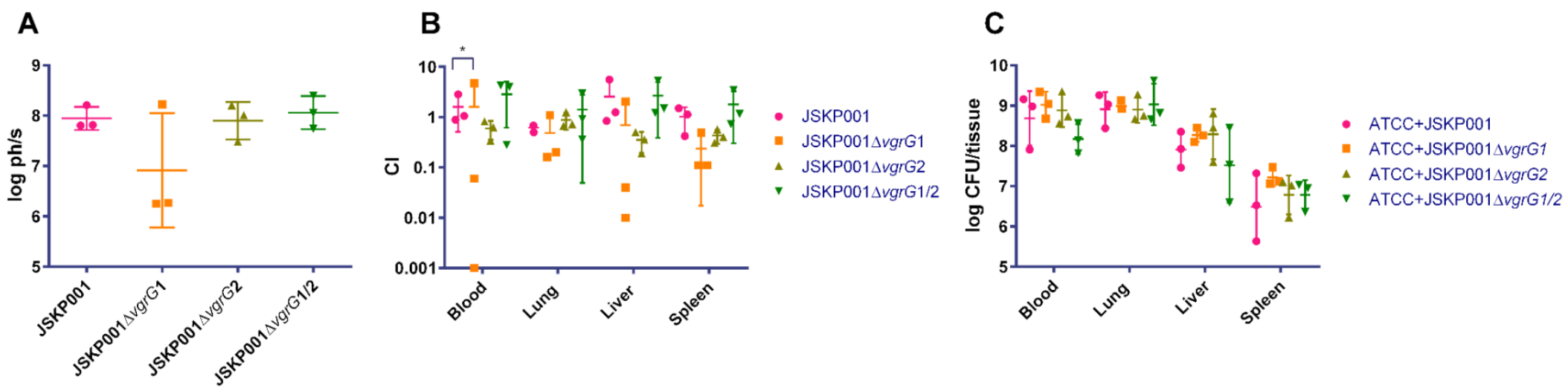

Figure 24. Fitness of the vgrG mutants in competition to the wild type strain. Albino C57BL/6J mice $(n=3)$ were co-infected with the ATCC 43816 with one of the $\operatorname{vgrG}$ mutants indicated at 1:1 ratio and animals were euthanized upon meeting a heart rate of $500 \mathrm{bpm}$ or below. (A) In vivo lung bacterial burdens of the luminescent JSKP001 strains estimated before euthanasia. (B) Competitive indexes of the JSKP001 strains in comparison to the ATCC 43816 strain in blood, lung, liver, and spleen. (C) Total bacterial burdens of the non-luminescent ATCC 43816 and the luminescent JSKP001 strains in blood, lung, liver, and spleen. 
We have demonstrated a potential role for T6SS cluster 1 for $K$. pneumoniae respiratory infection using competition infection model which will be followed by future investigations to confirm the proposed hypothesis. In addition to the importance of T6SSs in disease for other pathogens, these secretion systems play a role in bacterial competition (also known as bacterial dueling) [296, 298-302, 305]. Using the bioluminescent K. pneumoniae, we have observed frequent colonization of mice gut following the intratracheal administration of the bacteria. It is well known that mammalian gut is heavily colonized by many normal flora and therefore, $K$. pneumoniae might use its T6SSs to compete with other microbes in order to survive in that environment. The main mode of transmission of Klebsiella is fecal-oral route in which the bacteria spread in health care settings through the contaminated hands of patients, and health care providers due to unhygienic practices eventually leading to contamination of medical equipment $[23,223]$. Respiratory ventilators are one of the medical devices that become contaminated by the bacteria and $K$. pneumoniae is known to be a causative agent for VAP [39].

Thus, we would like to propose a model where K. pneumoniae spread from lungs of patients with respiratory disease into the gut where the bacteria uses its T6SS to compete with other microbes to thrive in that niche in addition to the possibility of acquiring antibiotic resistance genes followed by bacterial shedding in feces and without proper hygienic practices, medical devices become contaminated with $K$. pneumoniae leading to spread of the pulmonary 
disease to other hospitalized individuals (Figure 25). The proposed model is in consistence with the finding that the length of hospitalization is associated with higher possibility of acquiring K. pneumoniae infection [21].

One possible method of testing the proposed hypothesis is through infecting mice with bioluminescent wild type and the T6SS mutants using IMIT, monitoring development of the respiratory disease and bacterial dissemination to the gut using BLI, euthanizing animals at moribund endpoint, collecting their gut tissues, and estimating the amount of gut colonization by the T6SS mutants in comparison to the wild type bioluminescent strain using optical diagnostic imaging. If $K$. pneumoniae uses its T6SS to compete with other gut flora, then we would expect that deleting this secretion system will impact the ability of the bacteria to survive in the gut and if the proposed model was accurate then targeting T6SS would break the cycle of $K$. pneumoniae transmission. 


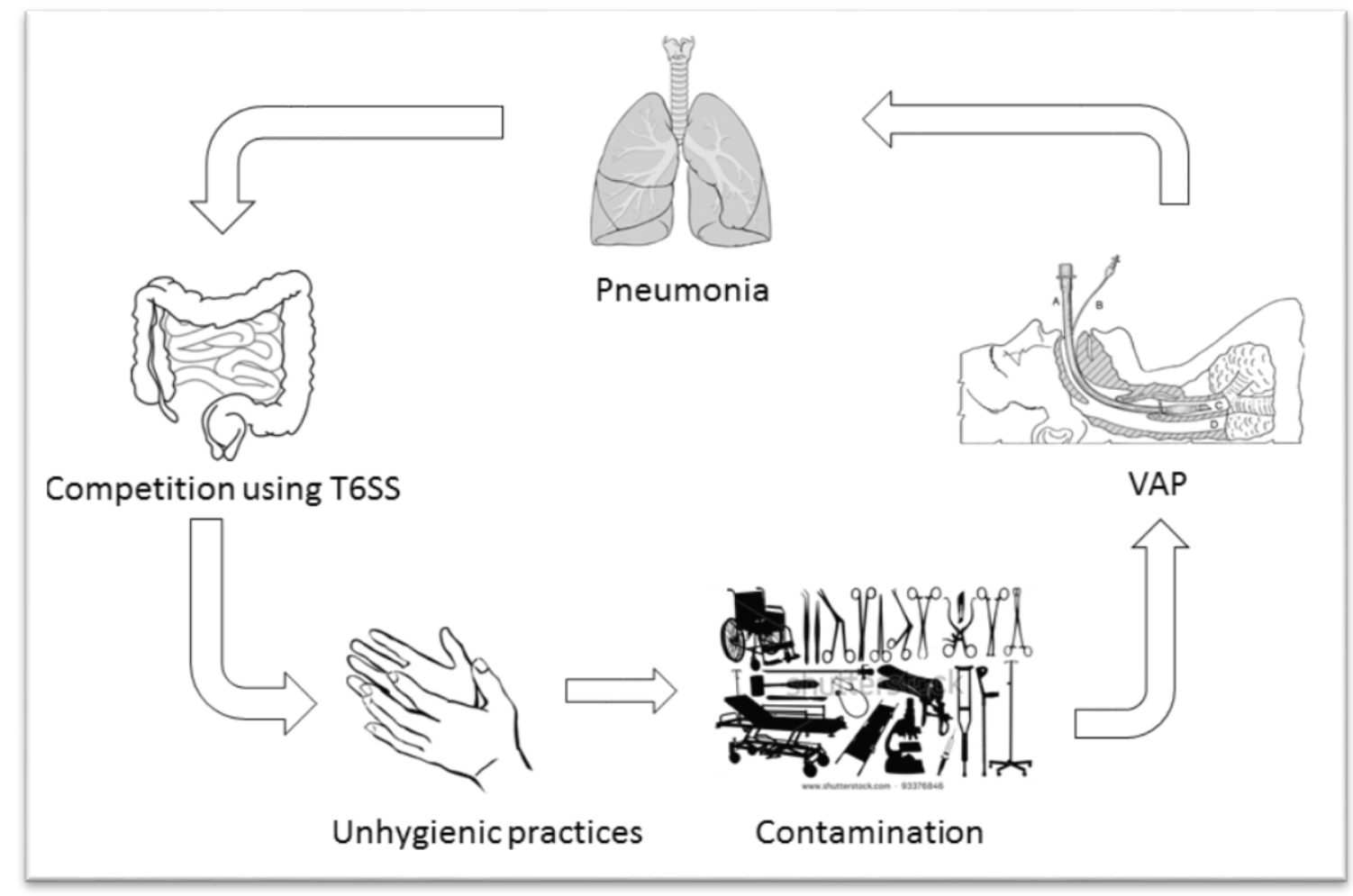

Figure 25. Proposed model of the indirect contribution of T6SS in $K$. pneumoniae nosocomial respiratory disease. K. pneumoniae disseminate from patient with pulmonary infection into the gut where the bacteria uses its T6SS to compete with other commensal microbes. The bacteria get shed into feces and without proper hands cleansing techniques, medical equipment become contaminated with $K$. pneumoniae. Contamination of ventilators or endotracheal Tubes leads to VAP in other hospitalized individuals. 


\subsection{The presentation of pulmonary disease using IMIT}

IMIT was developed and refined in K. pneumoniae before its application on in Burkholderia pseudomallei, the other respiratory pathogen that we study in our laboratory. In B. pseudomallei IMIT lead to the development of pulmonary infection in mice that resemble the human presentation of the disease more closely than intranasal infection model and such observation was made possible due to the development of a bioluminescent derivative of the bacteria in combination with in vivo imaging capability [306]. We do not know if the same phenomena take place in K. pneumoniae since we have started using IMIT since we started working with this pathogen in our laboratory. What we know for sure that using IMIT we can deliver $>98 \%$ of an inoculum into animals' lungs [187] and using this technique, we improved the specific delivery of $K$. pneumoniae into mice lungs as demonstrated by reduction in $\mathrm{LD}_{50}$ for the ATCC 438161 strain than what have been reported previously for BALB/c mice infected with intranasal route [99].

Introducing K. pneumoniae into mice lungs using IMIT somewhat resemble how an individual would acquire ventilator associated pneumoniae (VAP) in the hospital. Therefore, we speculate that IMIT recapitulate human disease more than other infection models such as the intranasal route frequently used in K. pneumoniae animal studies. The advantage of having bioluminescent K. pneumoniae strain that was generated in ATCC 43816 background, strain frequently used in biomedical research, will facilitate such comparison as it did 
with B. pseudomallei [306]. IMIT can also facilitate studies other than disease presentation upon infecting animals using different routes of infections.

This method of bacterial delivery would allow for examining host response to $K$. pneumoniae respiratory infection following activating host immune system using direct installation of an immune stimulating agent into mice lungs using IMIT. Using the same principle, IMIT would facilitate vaccine studies where the vaccine can be introduced directly into animals' lungs followed by bacterial administration. Since we can instill the bacteria directly into host lungs, we can use IMIT to identify factors required by K. pneumoniae to disseminate from the lungs and colonize other tissues. Such factors would be identified from the Tnseq analysis study that we have performed where dissemination defective mutants will be present in the lungs and missing from other anatomical sites. The confirmation of such defect will be achieved by deleting the predicted factors using targeted mutagenesis and then introduce the bacteria into the host lungs using IMIT and follow bacterial dissemination using bioluminescence imaging (BLI).

\subsection{The importance of intracellular life style for K. pneumoniae pathogenesis}

K. pneumoniae is considered as an extracellular pathogen but our data have demonstrated that this concept is not entirely true. In addition to the demonstration that $K$. pneumoniae strains are capable of replication within cultured macrophages [99], we have also established that a part of the bacterial 
inoculum become internalized within host cells in vivo following introducing the microbes into mice lungs. This phenomena raises the question whether this intracellular phenotype is something promoted by the bacteria as a way to evade host immune response or by the host as mechanism of protection against the invading pathogen. The fact that deleting the CPS increased internalization of the bacteria into host cells [99], suggest that $K$. pneumoniae resist uptake by host cells using that structure but that does not eliminate the possibility that the microbes might be promoting their own uptake since that the intact parental strains get internalized and even replicate within host cells.

The intracellular subpopulation of $K$. pneumoniae might get protected from host immune responses and from antimicrobial agents introduced into a host during therapy. Following the eradication of the extracellular population, the internalized bacteria might then serve as a reservoir to reestablish the pneumonic disease in the host. To test this hypothesis we can infect mice with the bioluminescent $K$. pneumoniae, allow the disease to develop for a short period of time, treat the animals with antimicrobial agents that work against extracellular microbes only, monitor the animals health status and BLI over a prolonged time points to track in they are going to undergo recurrent infection, and finally euthanize mice at different time points to test for the presence of intracellular bacteria. 


\subsection{Future implications for the bioluminescent $K$. pneumoniae}

In addition to the previously discussed implication for the bioluminescent

K. pneumoniae in distinguishing the respiratory disease presentation following inoculating the microbes through different routes, the JSKP001 will also facilitate other investigations. The bioluminescent strain will facilitate the performance of high throughput studies such as in vitro estimation of drugs $\mathrm{EC}_{50}$ and in vivo therapeutic efficacy such as the study that we conducted for testing meropenem direct effectiveness against $K$. pneumoniae respiratory disease. The same concept can be applied for testing the efficacy of other antimicrobial agents or assessing the efficacy of combinational therapy noninvasively.

In combination with IMIT, the bioluminescent $K$. pneumoniae will allow for the performance of multiple studies to improve our understating host response to the pulmonary disease. We have demonstrate that even with the very successful lung-specific bacterial delivery approach developed by our group [187], $\mathrm{K}$. pneumoniae undergo $\sim 1.3$ log variation in bacterial load by the $18 \mathrm{hr}$ post infection. This variation continue throughout the time course of the experiment in which animals that had higher colonization at the $18 \mathrm{hr}$ time point developed moribund disease more rapidly than those with lower bioluminescence signal. One explanation for such phenomena is that the host response to $K$. pneumoniae respiratory disease is variable in which some animals develop quicker response to the respiratory disease. We hypothesize that mice with less bacterial burdens early at disease might demonstrate earlier and higher levels of neutrophil recruitment known to be critical during the pneumonic disease [273]. 
Such hypothesis can be examined by infecting animals with the bioluminescent strain, monitor the development of the pneumonic disease using optical diagnostic imaging, and perform cytokine analysis profiles such as interleukin (IL)-8 and IL-17 at different time points during the course of the disease. We expect to detect differences in mice immune response in which animals with lower bacterial numbers early in disease might have higher proinflammatory cytokines levels indicative of early clearance which eventually the microbes overcome as they cause the full pneumonic disease. 


\section{REFERENCES}

1. Klebs, E., Beiträge zur Kenntniss der pathogenen Schistomyceten. Archiv für experimentelle Pathologie und Pharmakologie, 1875. 5(4): p. 350-377.

2. Friedlaender, C., Ueber die Schizomyceten bei der acuten fibrösen Pneumonie. Archiv für pathologische Anatomie und Physiologie und für klinische Medicin, 1882. 87(2): p. 319-324.

3. Austrian, R., The Gram stain and the etiology of lobar pneumonia, an historical note. Bacteriol Rev, 1960. 24(3): p. 261-5.

4. Zur Von-Frisch, A., Aetiologie des Rhinscleroms. Wien Med Wochenschr, 1882. 32: p. 96-7.

5. American Society for, M., Bergey's manual of determinative bacteriology. 1925, Baltimore: Williams \& Wilkins Co.

6. Gram, H.C., Ueber die isolierte Farbung der Schizomyceten in Schnittund Trocken-praparaten. Fortschritte der Medizin, 1884. 2: p. 185-89.

7. BRENNER, D.J., A.G. STEIGERWALT, and G.R. FANNING, Differentiation of Enterobacter aerogenes from Klebsiellae by Deoxyribonucleic Acid Reassociation. International Journal of Systematic and Evolutionary Microbiology, 1972. 22(4): p. 193-200.

8. Bergey, D.H., et al., Bergey's manual of determinative bacteriology. 1974, Baltimore: Williams \& Wilkins Co. 
9. Bagley, S.T., et al., Isolation of Klebsielleae from within living wood. Applied and Environmental Microbiology, 1978. 36(1): p. 178-185.

10. Brown, C. and R.J. Seidler, Potential pathogens in the environment: Klebsiella pneumoniae, a taxonomic and ecological enigma. Appl Microbiol, 1973. 25(6): p. 900-4.

11. Edberg, S.C., V. Piscitelli, and M. Cartter, Phenotypic characteristics of coliform and noncoliform bacteria from a public water supply compared with regional and national clinical species. Applied and Environmental Microbiology, 1986. 52(3): p. 474-478.

12. Matsen, J.M., J.A. Spindler, and R.O. Blosser, Characterization of Klebsiella isolates from natural receiving waters and comparison with human isolates. Appl Microbiol, 1974. 28(4): p. 672-8.

13. Seidler, R.J., M.D. Knittel, and C. Brown, Potential pathogens in the environment: cultural reactions and nucleic acid studies on Klebsiella pneumoniae from clinical and environmental sources. Appl Microbiol, 1975. 29(6): p. 819-25.

14. Podschun, R. and U. Ullmann, Klebsiella spp. as nosocomial pathogens: epidemiology, taxonomy, typing methods, and pathogenicity factors. Clin Microbiol Rev, 1998. 11(4): p. 589-603.

15. Fuxench-Lopez, Z. and C.H. Ramirez-Ronda, Pharyngeal flora in ambulatory alcoholic patients: prevalence of gram-negative bacilli. Arch Intern Med, 1978. 138(12): p. 1815-6. 
16. Kloos, W.E. and M.S. Musselwhite, Distribution and persistence of Staphylococcus and Micrococcus species and other aerobic bacteria on human skin. Appl Microbiol, 1975. 30(3): p. 381-5.

17. Casewell, M.W. and I. Phillips, Epidemiological patterns of Klebsiella colonization and infection in an intensive care ward. The Journal of Hygiene, 1978. 80(2): p. 295-300.

18. Casewell, M. and I. Phillips, Hands as route of transmission for Klebsiella species. British Medical Journal, 1977. 2(6098): p. 1315-1317.

19. Cooke, E.M., et al., Further studies on the sources of Klebsiella aerogenes in hospital patients. The Journal of Hygiene, 1979. 83(3): p. 391-395.

20. Pollack, M., et al., Factors influencing colonisation and antibioticresistance patterns of gram-negative bacteria in hospital patients. Lancet, 1972. 2(7779): p. 668-71.

21. Rose, H.D. and J. Schreier, The effect of hospitalization and antibiotic therapy on the gram-negative fecal flora. Am J Med Sci, 1968. 255: p. 228-36.

22. Selden, R., et al., Nosocomial klebsiella infections: intestinal colonization as a reservoir. Ann Intern Med, 1971. 74(5): p. 657-64.

23. Montgomerie, J.Z., Epidemiology of Klebsiella and hospital-associated infections. Rev Infect Dis, 1979. 1(5): p. 736-53.

24. Kuhn, I., et al., The use of colonization rate and epidemic index as tools to illustrate the epidemiology of faecal Enterobacteriaceae strains in Swedish neonatal wards. J Hosp Infect, 1993. 23(4): p. 287-97. 
25. Hart, C.A., Klebsiellae and neonates. J Hosp Infect, 1993. 23(2): p. 83-6.

26. Doebbeling, B.N., Epidemics: identification and management. Prevention and control of nosocomial infections, 1993: p. 177-206.

27. Stamm, W.E., R.A. Weinstein, and R.E. Dixon, Comparison of endemic and epidemic nosocomial infections. Am J Med, 1981. 70(2): p. 393-7.

28. Ko, W.C., et al., Community-acquired Klebsiella pneumoniae bacteremia: global differences in clinical patterns. Emerg Infect Dis, 2002. 8(2): p. 1606.

29. Tsay, R.W., et al., Characteristics of bacteremia between communityacquired and nosocomial Klebsiella pneumoniae infection: risk factor for mortality and the impact of capsular serotypes as a herald for communityacquired infection. Arch Intern Med, 2002. 162(9): p. 1021-7.

30. Kang, C.I., et al., Community-acquired versus nosocomial Klebsiella pneumoniae bacteremia: clinical features, treatment outcomes, and clinical implication of antimicrobial resistance. J Korean Med Sci, 2006. 21(5): p. 816-22.

31. Lee, K.H., et al., Klebsiella bacteraemia: a report of 101 cases from National University Hospital, Singapore. J Hosp Infect, 1994. 27(4): p. 299-305.

32. Feldman, C., et al., Klebsiella pneumoniae bacteraemia at an urban general hospital. J Infect, 1990. 20(1): p. 21-31. 
33. Lu, C.H., W.N. Chang, and H.S. Wu, Klebsiella pneumoniae meningitis: analysis on clinical features of thirty-two adult patients. Zhonghua Yi Xue Za Zhi (Taipei), 1997. 60(6): p. 296-302.

34. Watanakunakorn, C. and J. Jura, Klebsiella bacteremia: a review of 196 episodes during a decade (1980-1989). Scand J Infect Dis, 1991. 23(4): p. 399-405.

35. Hansen, D.S., A. Gottschau, and H.J. Kolmos, Epidemiology of Klebsiella bacteraemia: a case control study using Escherichia coli bacteraemia as control. J Hosp Infect, 1998. 38(2): p. 119-32.

36. Haddy, R.I., et al., Klebsiella pneumoniae bacteremia in the community hospital. J Fam Pract, 1989. 28(6): p. 686-90.

37. Lin, S.H., et al., Sputum bacteriology in hospitalized patients with acute exacerbation of chronic obstructive pulmonary disease in Taiwan with an emphasis on Klebsiella pneumoniae and Pseudomonas aeruginosa. Respirology, 2007. 12(1): p. 81-7.

38. Yi, Z.M., H. Yang, and X.D. Xiang, [Characteristics of bacteriology and drug sensitivity in patients with COPD combined with pneumonia]. Hunan Yi Ke Da Xue Xue Bao, 2003. 28(3): p. 272-4.

39. Kollef, M.H., et al., Epidemiology and outcomes of health-care-associated pneumonia: results from a large US database of culture-positive pneumonia. Chest, 2005. 128(6): p. 3854-62.

40. Prince, S.E.S., Klebsiella pneumoniae pneumonia. Heart and Lung: The Journal of Acute and Critical Care, 1997. 26(5): p. 413-7. 
41. Yu, W.L. and Y.C. Chuang. Clinical features, diagnosis, and treatment of Klebsiella pneumoniae infection. 2015 [cited 201612 Mar ]; Available from: http://www.uptodate.com.echo.louisville.edu/contents/clinicalfeatures-diagnosis-and-treatment-of-klebsiella-pneumoniaeinfection?source=search result\&search=klebsiella\&selectedTitle=1 150\# $\underline{\mathrm{H} 11}$.

42. Okada, F., et al., Clinical and pulmonary thin-section CT findings in acute Klebsiella pneumoniae pneumonia. Eur Radiol, 2009. 19(4): p. 809-15.

43. Mandell, L.A., et al., Infectious Diseases Society of America/American Thoracic Society consensus guidelines on the management of communityacquired pneumonia in adults. Clin Infect Dis, 2007. 44 Suppl 2: p. S2772.

44. Radyowijati, A. and H. Haak, Improving antibiotic use in low-income countries: an overview of evidence on determinants. Soc Sci Med, 2003. 57(4): p. 733-44.

45. Lin, Y.-T., et al., Community-onset Klebsiella pneumoniae pneumonia in Taiwan: clinical features of the disease and associated microbiological characteristics of isolates from pneumonia and nasopharynx. Frontiers in Microbiology, 2015. 6.

46. Hu, H.C., et al., Outcome analysis of patients requiring mechanical ventilation with severe community-acquired pneumonia and identified bacterial pathogens. Chang Gung Med J, 2005. 28(4): p. 229-36. 
47. Marik, P.E., The clinical features of severe community-acquired pneumonia presenting as septic shock. Norasept II Study Investigators. J Crit Care, 2000. 15(3): p. 85-90.

48. Paganin, F., et al., Severe community-acquired pneumonia: assessment of microbial aetiology as mortality factor. Eur Respir J, 2004. 24(5): p. 77985.

49. Fiberesima, F.P. and A.C. Onwuchekwa, Community acquired pneumonia in Port Harcourt Rivers State of Nigeria. Cent Afr J Med, 2008. 54(1-4): $p$. $1-8$.

50. Lin, Y.T., et al., Bacteremic community-acquired pneumonia due to Klebsiella pneumoniae: clinical and microbiological characteristics in Taiwan, 2001-2008. BMC Infect Dis, 2010. 10: p. 307.

51. Hammond, J.M., et al., Intensive care management of communityacquired Klebsiella pneumoniae. Respir Med, 1991. 85(1): p. 11-6.

52. Knight, L., R.G. Fraser, and H.G. Robson, Massive pulmonary gangrene: a severe complication of Klebsiella pneumonia. Can Med Assoc J, 1975. 112(2): p. 196-8.

53. Moon, W.K., et al., Complications of Klebsiella pneumonia: CT evaluation. J Comput Assist Tomogr, 1995. 19(2): p. 176-81.

54. Paterson, D.L., L.K. Siu, and F.Y. Chang. Klebsiella species (K. pneumoniae, K. oxytoca, K. ozaenae and K. rhinoscleromatis). 2014 [cited 2016; Available from: http://www.antimicrobe.org/new/b107.asp. 
55. Hoffman, N.R. and F.S. Preston, Jr., Friedlander's pneumonia. A report of 11 cases and appraisal of antibiotic therapy. Dis Chest, 1968. 53(4): p. $481-6$.

56. Jong, G.M., et al., Rapidly fatal outcome of bacteremic Klebsiella pneumoniae pneumonia in alcoholics. Chest, 1995. 107(1): p. 214-7.

57. Karnad, A., S. Alvarez, and S.L. Berk, Pneumonia caused by gramnegative bacilli. Am J Med, 1985. 79(1a): p. 61-7.

58. Kashuba, A.D., et al., Optimizing aminoglycoside therapy for nosocomial pneumonia caused by gram-negative bacteria. Antimicrob Agents Chemother, 1999. 43(3): p. 623-9.

59. Brown, R.B., et al., Double-blind study of endotracheal tobramycin in the treatment of gram-negative bacterial pneumonia. The Endotracheal Tobramycin Study Group. Antimicrob Agents Chemother, 1990. 34(2): p. $269-72$.

60. Gentry, L.O., et al., Parenteral followed by oral ofloxacin for nosocomial pneumonia and community-acquired pneumonia requiring hospitalization. Am Rev Respir Dis, 1992. 145(1): p. 31-5.

61. Trenholme, G.M., et al., Randomized study of intravenous/oral ciprofloxacin versus ceftazidime in the treatment of hospital and nursing home patients with lower respiratory tract infections. Am J Med, 1989. 87(5a): p. $116 \mathrm{~s}-118 \mathrm{~s}$.

62. Paterson, D.L., et al., International prospective study of Klebsiella pneumoniae bacteremia: implications of extended-spectrum beta- 
lactamase production in nosocomial Infections. Ann Intern Med, 2004. 140(1): p. 26-32.

63. Cartelle, M., et al., Risk factors for colonization and infection in a hospital outbreak caused by a strain of Klebsiella pneumoniae with reduced susceptibility to expanded-spectrum cephalosporins. J Clin Microbiol, 2004. 42(9): p. 4242-9.

64. DiPersio, J.R., et al., Evolution and dissemination of extended-spectrum beta-lactamase-producing Klebsiella pneumoniae: epidemiology and molecular report from the SENTRY Antimicrobial Surveillance Program (1997-2003). Diagn Microbiol Infect Dis, 2005. 51(1): p. 1-7.

65. Wiener, J., et al., Multiple antibiotic-resistant Klebsiella and Escherichia coli in nursing homes. Jama, 1999. 281(6): p. 517-23.

66. Dinubile, M.J., et al., Bowel colonization with resistant gram-negative bacilli after antimicrobial therapy of intra-abdominal infections: observations from two randomized comparative clinical trials of ertapenem therapy. Eur J Clin Microbiol Infect Dis, 2005. 24(7): p. 443-9.

67. Tumbarello, M., et al., Bloodstream infections caused by extendedspectrum-beta-lactamase-producing Klebsiella pneumoniae: risk factors, molecular epidemiology, and clinical outcome. Antimicrob Agents Chemother, 2006. 50(2): p. 498-504.

68. Garcia San Miguel, L., et al., Clinical variables associated with the isolation of Klebsiella pneumoniae expressing different extended-spectrum beta-lactamases. Clin Microbiol Infect, 2007. 13(5): p. 532-8. 
69. Herbert, S., et al., Large outbreak of infection and colonization with gramnegative pathogens carrying the metallo- beta -lactamase gene blalMP-4 at a 320-bed tertiary hospital in Australia. Infect Control Hosp Epidemiol, 2007. 28(1): p. 98-101.

70. Nouer, S.A., et al., Risk factors for acquisition of multidrug-resistant Pseudomonas aeruginosa producing SPM metallo-beta-lactamase. Antimicrob Agents Chemother, 2005. 49(9): p. 3663-7.

71. Schwaber, M.J., et al., Predictors of carbapenem-resistant Klebsiella pneumoniae acquisition among hospitalized adults and effect of acquisition on mortality. Antimicrob Agents Chemother, 2008. 52(3): p. 1028-33.

72. Zarkotou, O., et al., Predictors of mortality in patients with bloodstream infections caused by KPC-producing Klebsiella pneumoniae and impact of appropriate antimicrobial treatment. Clin Microbiol Infect, 2011. 17(12): p. 1798-803.

73. Walsh, T.R., et al., Metallo-beta-lactamases: the quiet before the storm? Clin Microbiol Rev, 2005. 18(2): p. 306-25.

74. Hirakata, Y., et al., Rapid detection and evaluation of clinical characteristics of emerging multiple-drug-resistant gram-negative rods carrying the metallo-beta-lactamase gene blalMP. Antimicrob Agents Chemother, 1998. 42(8): p. 2006-11. 
75. Bratu, S., et al., Carbapenemase-producing Klebsiella pneumoniae in Brooklyn, NY: molecular epidemiology and in vitro activity of polymyxin $B$ and other agents. J Antimicrob Chemother, 2005. 56(1): p. 128-32.

76. Ruiz, E., et al., Acquisition of carbapenem resistance in multiresistant Klebsiella pneumoniae strains harbouring blaCTX-M-15, qnrS1 and aac(6')-Ib-cr genes. J Med Microbiol, 2012. 61(Pt 5): p. 672-7.

77. Kaczmarek, F.M., et al., High-level carbapenem resistance in a Klebsiella pneumoniae clinical isolate is due to the combination of bla(ACT-1) betalactamase production, porin OmpK35/36 insertional inactivation, and down-regulation of the phosphate transport porin phoe. Antimicrob Agents Chemother, 2006. 50(10): p. 3396-406.

78. Song, W., et al., In vivo selection of carbapenem-resistant Klebsiella pneumoniae by OmpK36 loss during meropenem treatment. Diagn Microbiol Infect Dis, 2009. 65(4): p. 447-9.

79. Paterson, D.L., Recommendation for treatment of severe infections caused by Enterobacteriaceae producing extended-spectrum betalactamases (ESBLs). Clin Microbiol Infect, 2000. 6(9): p. 460-3.

80. Meyer, K.S., et al., Nosocomial outbreak of Klebsiella infection resistant to late-generation cephalosporins. Ann Intern Med, 1993. 119(5): p. 353-8.

81. Paterson, D.L., et al., Antibiotic therapy for Klebsiella pneumoniae bacteremia: implications of production of extended-spectrum betalactamases. Clin Infect Dis, 2004. 39(1): p. 31-7. 
82. Paterson, D.L., et al., Epidemiology of ciprofloxacin resistance and its relationship to extended-spectrum beta-lactamase production in Klebsiella pneumoniae isolates causing bacteremia. Clin Infect Dis, 2000. 30(3): p. 473-8.

83. Thomson, K.S. and E.S. Moland, Cefepime, piperacillin-tazobactam, and the inoculum effect in tests with extended-spectrum beta-lactamaseproducing Enterobacteriaceae. Antimicrob Agents Chemother, 2001. 45(12): p. 3548-54.

84. Paterson, D.L., et al., Fatal infection due to extended-spectrum betalactamase-producing Escherichia coli: implications for antibiotic choice for spontaneous bacterial peritonitis. Clin Infect Dis, 1999. 28(3): p. 683-4.

85. Pillay, T., et al., Piperacillin/tazobactam in the treatment of Klebsiella pneumoniae infections in neonates. Am J Perinatol, 1998. 15(1): p. 47-51.

86. Szabo, D., et al., In vitro and in vivo activities of amikacin, cefepime, amikacin plus cefepime, and imipenem against an SHV-5 extendedspectrum beta-lactamase-producing Klebsiella pneumoniae strain. Antimicrob Agents Chemother, 2001. 45(4): p. 1287-91.

87. Harada, Y., et al., In vitro and in vivo activities of piperacillin-tazobactam and meropenem at different inoculum sizes of ESBL-producing Klebsiella pneumoniae. Clin Microbiol Infect, 2014. 20(11): p. O831-9.

88. Paterson, D.L., et al., Outcome of cephalosporin treatment for serious infections due to apparently susceptible organisms producing extended- 
spectrum beta-lactamases: implications for the clinical microbiology laboratory. J Clin Microbiol, 2001. 39(6): p. 2206-12.

89. Zavascki, A.P., J.B. Bulitta, and C.B. Landersdorfer, Combination therapy for carbapenem-resistant Gram-negative bacteria. Expert Rev Anti Infect Ther, 2013. 11(12): p. 1333-53.

90. Tzouvelekis, L.S., et al., Treating infections caused by carbapenemaseproducing Enterobacteriaceae. Clin Microbiol Infect, 2014. 20(9): p. 86272.

91. Morrill, H.J., et al., Treatment Options for Carbapenem-Resistant Enterobacteriaceae Infections. Open Forum Infectious Diseases, 2015. 2(2): p. ofv050.

92. Lawlor, M.S., et al., Identification of Klebsiella pneumoniae virulence determinants using an intranasal infection model. Mol Microbiol, 2005. 58(4): p. 1054-73.

93. Chou, H.C., et al., Isolation of a chromosomal region of Klebsiella pneumoniae associated with allantoin metabolism and liver infection. Infect Immun, 2004. 72(7): p. 3783-92.

94. Held, T.K., et al., Granulocyte colony-stimulating factor worsens the outcome of experimental Klebsiella pneumoniae pneumonia through direct interaction with the bacteria. Blood, 1998. 91(7): p. 2525-35.

95. Lau, H.Y., S. Clegg, and T.A. Moore, Identification of Klebsiella pneumoniae genes uniquely expressed in a strain virulent using a murine model of bacterial pneumonia. Microb Pathog, 2007. 42(4): p. 148-55. 
96. Tu, Y.C., et al., Genetic requirements for Klebsiella pneumoniae-induced liver abscess in an oral infection model. Infect Immun, 2009. 77(7): p. 2657-71.

97. Hershman, M.J., et al., Modulation of Klebsiella pneumoniae infection of mice by interferon-gamma. Clin Exp Immunol, 1988. 72(3): p. 406-9.

98. Rosen, D.A., et al., Utilization of an intracellular bacterial community pathway in Klebsiella pneumoniae urinary tract infection and the effects of FimK on type 1 pilus expression. Infect Immun, 2008. 76(7): p. 3337-45.

99. Fodah, R.A., et al., Correlation of Klebsiella pneumoniae comparative genetic analyses with virulence profiles in a murine respiratory disease model. PLoS One, 2014. 9(9): p. e107394.

100. Bachman, M.A., et al., Genome-Wide Identification of Klebsiella pneumoniae Fitness Genes during Lung Infection. mBio, 2015. 6(3): p. e00775-15.

101. Lawlor, M.S., S.A. Handley, and V.L. Miller, Comparison of the host responses to wild-type and cpsB mutant Klebsiella pneumoniae infections. Infect Immun, 2006. 74(9): p. 5402-7.

102. Keller, C.E., et al., Susceptibility of irradiated B6D2F1/J mice to Klebsiella pneumoniae administered intratracheally: a pulmonary infection model in an immunocompromised host. Comp Med, 2003. 53(4): p. 397-403.

103. Moore, T.A., et al., Divergent Role of Gamma Interferon in a Murine Model of Pulmonary versus Systemic Klebsiella pneumoniae Infection. Infection and Immunity, 2002. 70(11): p. 6310-6318. 
104. Berendt, R.F., G.G. Long, and J.S. Walker, Treatment of respiratory Klebsiella pneumoniae infection in mice with aerosols of kanamycin. Antimicrob Agents Chemother, 1975. 8(5): p. 585-90.

105. Hackstein, H., et al., Modulation of respiratory dendritic cells during Klebsiella pneumonia infection. Respir Res, 2013. 14: p. 91.

106. Mehrad, B. and T.J. Standiford, Use of Animal Models in the Study of Inflammatory Mediators of Pneumonia. Ilar j, 1999. 40(4): p. 167-174.

107. Yoshida, K., et al., Protection against pulmonary infection with Klebsiella pneumoniae in mice by interferon-gamma through activation of phagocytic cells and stimulation of production of other cytokines. J Med Microbiol, 2001. 50(11): p. 959-64.

108. Greenberger, M.J., et al., Neutralization of IL-10 increases survival in a murine model of Klebsiella pneumonia. J Immunol, 1995. 155(2): p. 722-9.

109. de Stoppelaar, S.F., et al., Thrombocytopenia impairs host defense in gram-negative pneumonia-derived sepsis in mice. Blood, 2014. 124(25): p. 3781-90.

110. Green, G.M., et al., Defense mechanisms of the respiratory membrane. Am Rev Respir Dis, 1977. 115(3): p. 479-514.

111. Sutherland, R.E., et al., Mast Cell IL-6 Improves Survival From Klebsiella Pneumonia and Sepsis by Enhancing Neutrophil Killing. Journal of immunology (Baltimore, Md. : 1950), 2008. 181(8): p. 5598-5605.

112. Happel, K.I., et al., Divergent roles of IL-23 and IL-12 in host defense against Klebsiella pneumoniae. J Exp Med, 2005. 202(6): p. 761-9. 
113. Ye, P., et al., Requirement of interleukin 17 receptor signaling for lung CXC chemokine and granulocyte colony-stimulating factor expression, neutrophil recruitment, and host defense. J Exp Med, 2001. 194(4): p. $519-27$.

114. Bachman, M.A., et al., Klebsiella pneumoniae yersiniabactin promotes respiratory tract infection through evasion of lipocalin 2. Infect Immun, 2011. 79(8): p. 3309-16.

115. Wieland, C.W., et al., Host defence during Klebsiella pneumonia relies on haematopoietic-expressed Toll-like receptors 4 and 2. Eur Respir J, 2011. 37(4): p. 848-57.

116. Tsai, W.C., et al., Lung-specific transgenic expression of KC enhances resistance to Klebsiella pneumoniae in mice. J Immunol, 1998. 161(5): p. 2435-40.

117. McColm, A.A., et al., Evaluation of ceftazidime in experimental Klebsiella pneumoniae pneumonia: comparison with other antibiotics and measurement of its penetration into respiratory tissues and secretions. $\mathrm{J}$ Antimicrob Chemother, 1986. 18(5): p. 599-608.

118. Hilliard, J.J., et al., Comparative effects of carbapenems on bacterial load and host immune response in a Klebsiella pneumoniae murine pneumonia model. Antimicrob Agents Chemother, 2011. 55(2): p. 836-44.

119. Hirsch, E.B., et al., Assessment of Antimicrobial Combinations for Klebsiella pneumoniae Carbapenemase-Producing K. pneumoniae. Journal of Infectious Diseases, 2012. 
120. Saini, A., S. Sharma, and S. Chhibber, Protective efficacy of Emblica officinalis against Klebsiella pneumoniae induced pneumonia in mice. Indian J Med Res, 2008. 128(2): p. 188-93.

121. Sharma, S., et al., Dietary supplementation with omega-3 polyunsaturated fatty acids ameliorates acute pneumonia induced by Klebsiella pneumoniae in BALB/c mice. Can J Microbiol, 2013. 59(7): p. 503-10.

122. Tan, S., et al., A novel chemosynthetic peptide with beta-sheet motif efficiently kills Klebsiella pneumoniae in a mouse model. Int $\mathrm{J}$ Nanomedicine, 2015. 10: p. 1045-59.

123. Bansal, S., K. Harjai, and S. Chhibber, Depolymerase improves gentamicin efficacy during Klebsiella pneumoniae induced murine infection. BMC Infect Dis, 2014. 14: p. 456.

124. Cao, F., et al., Evaluation of the Efficacy of a Bacteriophage in the Treatment of Pneumonia Induced by Multidrug Resistance Klebsiella pneumoniae in Mice. BioMed Research International, 2015. 2015: p. 9.

125. Regueiro, V., et al., The uptake of a Klebsiella pneumoniaecapsule polysaccharide mutant triggers an inflammatory response by human airway epithelial cells. Microbiology, 2006. 152.

126. Brisse, S., S. Issenhuth-Jeanjean, and P.A.D. Grimont, Molecular Serotyping of Klebsiella Species Isolates by Restriction of the Amplified Capsular Antigen Gene Cluster. Journal of Clinical Microbiology, 2004. 42(8): p. 3388-3398. 
127. Orskov and F. Orskov, SEROTYPING OF KLEBSIELLA. METHODS IN MICROBIOLOGY, 1984. 14: p. 143-164.

128. Cryz, S.J., Jr., et al., Seroepidemiology of Klebsiella bacteremic isolates and implications for vaccine development. J Clin Microbiol, 1986. 23(4): p. $687-90$.

129. Fung, C.P., et al., A 5-year study of the seroepidemiology of Klebsiella pneumoniae: high prevalence of capsular serotype $\mathrm{K} 1$ in Taiwan and implication for vaccine efficacy. J Infect Dis, 2000. 181(6): p. 2075-9.

130. Fung, C.P., et al., A global emerging disease of Klebsiella pneumoniae liver abscess: is serotype $\mathrm{K} 1$ an important factor for complicated endophthalmitis? Gut, 2002. 50(3): p. 420-4.

131. Yeh, K.M., et al., Capsular serotype $K 1$ or K2, rather than magA and rmpA, is a major virulence determinant for Klebsiella pneumoniae liver abscess in Singapore and Taiwan. J Clin Microbiol, 2007. 45(2): p. 46671.

132. Fang, C.T., et al., Klebsiella pneumoniae genotype K1: an emerging pathogen that causes septic ocular or central nervous system complications from pyogenic liver abscess. Clin Infect Dis, 2007. 45(3): $p$. 284-93.

133. Chung, D.R., et al., Emerging invasive liver abscess caused by K1 serotype Klebsiella pneumoniae in Korea. J Infect, 2007. 54(6): p. 578-83. 
134. Jenney, A.W., et al., Seroepidemiology of Klebsiella pneumoniae in an Australian Tertiary Hospital and its implications for vaccine development. J Clin Microbiol, 2006. 44(1): p. 102-7.

135. Blanchette, E.A. and S.J. Rubin, Seroepidemiology of clinical isolates of Klebsiella in Connecticut. J Clin Microbiol, 1980. 11(5): p. 474-8.

136. Mizuta, K., et al., Virulence for mice of Klebsiella strains belonging to the O1 group: relationship to their capsular (K) types. Infect Immun, 1983. 40(1): p. 56-61.

137. Athamna, A., et al., Lectinophagocytosis of encapsulated Klebsiella pneumoniae mediated by surface lectins of guinea pig alveolar macrophages and human monocyte-derived macrophages. Infect Immun, 1991. 59(5): p. 1673-82.

138. Ofek, I., et al., Nonopsonic phagocytosis of microorganisms. Annu Rev Microbiol, 1995. 49: p. 239-76.

139. Kabha, K., et al., Relationships among capsular structure, phagocytosis, and mouse virulence in Klebsiella pneumoniae. Infect Immun, 1995. 63(3): p. 847-52.

140. Lin, J.C., et al., High prevalence of phagocytic-resistant capsular serotypes of Klebsiella pneumoniae in liver abscess. Microbes Infect, 2004. 6(13): p. 1191-8.

141. Sahly, H., et al., Impairment of respiratory burst in polymorphonuclear leukocytes by extended-spectrum beta-lactamase-producing strains of 
Klebsiella pneumoniae. Eur J Clin Microbiol Infect Dis, 2004. 23(1): p. 206.

142. Kawai, T., Hypermucoviscosity: an extremely sticky phenotype of Klebsiella pneumoniae associated with emerging destructive tissue abscess syndrome. Clin Infect Dis, 2006. 42(10): p. 1359-61.

143. Fang, C.T., et al., A novel virulence gene in Klebsiella pneumoniae strains causing primary liver abscess and septic metastatic complications. J Exp Med, 2004. 199(5): p. 697-705.

144. Shon, A.S., R.P. Bajwa, and T.A. Russo, Hypervirulent (hypermucoviscous) Klebsiella pneumoniae: a new and dangerous breed. Virulence, 2013. 4(2): p. 107-18.

145. Alvarez, D., et al., Capsular polysaccharide is a major complement resistance factor in lipopolysaccharide O side chain-deficient Klebsiella pneumoniae clinical isolates. Infect Immun, 2000. 68(2): p. 953-5.

146. Yu, W.L., et al., Association between rmpA and magA genes and clinical syndromes caused by Klebsiella pneumoniae in Taiwan. Clin Infect Dis, 2006. 42(10): p. 1351-8.

147. Lee, H.C., et al., Clinical implications of hypermucoviscosity phenotype in Klebsiella pneumoniae isolates: association with invasive syndrome in patients with community-acquired bacteraemia. J Intern Med, 2006. 259(6): p. 606-14. 
148. Nadasy, K.A., R. Domiati-Saad, and M.A. Tribble, Invasive Klebsiella pneumoniae syndrome in North America. Clin Infect Dis, 2007. 45(3): p. e25-8.

149. Liao, P.Y., et al., Rapidly fatal gas-forming pyogenic psoas abscess caused by Klebsiella pneumoniae. Clin Infect Dis, 2007. 44(9): p. 1253-5.

150. Ku, Y.H., Y.C. Chuang, and W.L. Yu, Clinical spectrum and molecular characteristics of Klebsiella pneumoniae causing community-acquired extrahepatic abscess. J Microbiol Immunol Infect, 2008. 41(4): p. 311-7.

151. Tomas, J.M., et al., Role of capsule and O antigen in resistance of Klebsiella pneumoniae to serum bactericidal activity. Infect Immun, 1986. 54(1): p. 85-9.

152. McCallum, K.L., D.H. Laakso, and C. Whitfield, Use of a bacteriophageencoded glycanase enzyme in the generation of lipopolysaccharide $O$ side chain deficient mutants of Escherichia coli O9:K30 and Klebsiella 01:K20: role of $\mathrm{O}$ and $\mathrm{K}$ antigens in resistance to complement-mediated serum killing. Can J Microbiol, 1989. 35(11): p. 994-9.

153. Alberti, S., et al., C1q binding and activation of the complement classical pathway by Klebsiella pneumoniae outer membrane proteins. Infect Immun, 1993. 61(3): p. 852-60.

154. Alberti, S., et al., Analysis of complement C3 deposition and degradation on Klebsiella pneumoniae. Infect Immun, 1996. 64(11): p. 4726-32. 
155. Shankar-Sinha, S., et al., The Klebsiella pneumoniae $O$ antigen contributes to bacteremia and lethality during murine pneumonia. Infect Immun, 2004. 72(3): p. 1423-30.

156. Izquierdo, L., et al., The Klebsiella pneumoniae wabG gene: role in biosynthesis of the core lipopolysaccharide and virulence. J Bacteriol, 2003. 185(24): p. 7213-21.

157. Cortes, G., et al., Molecular analysis of the contribution of the capsular polysaccharide and the lipopolysaccharide $O$ side chain to the virulence of Klebsiella pneumoniae in a murine model of pneumonia. Infect Immun, 2002. 70(5): p. 2583-90.

158. Chu, B.C., et al., Siderophore uptake in bacteria and the battle for iron with the host; a bird's eye view. Biometals, 2010. 23(4): p. 601-11.

159. Lawlor, M.S., C. O'Connor, and V.L. Miller, Yersiniabactin Is a Virulence Factor for Klebsiella pneumoniae during Pulmonary Infection. Infection and Immunity, 2007. 75(3): p. 1463-1472.

160. Miles, A.A. and P.L. Khimji, Enterobacterial chelators of iron: their occurrence, detection, and relation to pathogenicity. J Med Microbiol, 1975. 8(4): p. 477-90.

161. Lodge, J.M., P. Williams, and M.R. Brown, Influence of growth rate and iron limitation on the expression of outer membrane proteins and enterobactin by Klebsiella pneumoniae grown in continuous culture. J Bacteriol, 1986. 165(2): p. 353-6. 
162. Nassif, X. and P.J. Sansonetti, Correlation of the virulence of Klebsiella pneumoniae $\mathrm{K} 1$ and $\mathrm{K} 2$ with the presence of a plasmid encoding aerobactin. Infect Immun, 1986. 54(3): p. 603-8.

163. Podschun, R., et al., Serotypes, hemagglutinins, siderophore synthesis, and serum resistance of Klebsiella isolates causing human urinary tract infections. J Infect Dis, 1993. 168(6): p. 1415-21.

164. Podschun, R., A. Fischer, and U. Ullmann, Siderophore production of Klebsiella species isolated from different sources. Zentralbl Bakteriol, 1992. 276(4): p. 481-6.

165. Tarkkanen, A.M., et al., Fimbriation, capsulation, and iron-scavenging systems of Klebsiella strains associated with human urinary tract infection. Infect Immun, 1992. 60(3): p. 1187-92.

166. Vernet, V., et al., Virulence factors (aerobactin and mucoid phenotype) in Klebsiella pneumoniae and Escherichia coli blood culture isolates. FEMS Microbiol Lett, 1995. 130(1): p. 51-7.

167. Russo, T.A., et al., Hypervirulent K. pneumoniae secretes more and more active iron-acquisition molecules than "classical" K. pneumoniae thereby enhancing its virulence. PLoS One, 2011. 6(10): p. e26734.

168. Russo, T.A., et al., Aerobactin mediates virulence and accounts for increased siderophore production under iron-limiting conditions by hypervirulent (hypermucoviscous) Klebsiella pneumoniae. Infect Immun, 2014. 82(6): p. 2356-67. 
169. Russo, T.A., et al., Aerobactin, but not yersiniabactin, salmochelin, or enterobactin, enables the growth/survival of hypervirulent (hypermucoviscous) Klebsiella pneumoniae ex vivo and in vivo. Infect Immun, 2015. 83(8): p. 3325-33.

170. Bachman, M.A., et al., Interaction of lipocalin 2, transferrin, and siderophores determines the replicative niche of Klebsiella pneumoniae during pneumonia. MBio, 2012. 3(6).

171. Gerlach, G.F., S. Clegg, and B.L. Allen, Identification and characterization of the genes encoding the type 3 and type 1 fimbrial adhesins of Klebsiella pneumoniae. J Bacteriol, 1989. 171(3): p. 1262-70.

172. Jones, C.H., et al., FimH adhesin of type 1 pili is assembled into a fibrillar tip structure in the Enterobacteriaceae. Proc Natl Acad Sci U S A, 1995. 92(6): p. 2081-5.

173. Sebghati, T.A., et al., Characterization of the type 3 fimbrial adhesins of Klebsiella strains. Infect Immun, 1998. 66(6): p. 2887-94.

174. Struve, C., M. Bojer, and K.A. Krogfelt, Identification of a Conserved Chromosomal Region Encoding Klebsiella pneumoniae Type 1 and Type 3 Fimbriae and Assessment of the Role of Fimbriae in Pathogenicity. Infection and Immunity, 2009. 77(11): p. 5016-5024.

175. Struve, C., M. Bojer, and K.A. Krogfelt, Characterization of Klebsiella pneumoniae type 1 fimbriae by detection of phase variation during colonization and infection and impact on virulence. Infect Immun, 2008. 76(9): p. 4055-65. 
176. Jagnow, J. and S. Clegg, Klebsiella pneumoniae MrkD-mediated biofilm formation on extracellular matrix- and collagen-coated surfaces. Microbiology, 2003. 149(Pt 9): p. 2397-405.

177. Langstraat, J., M. Bohse, and S. Clegg, Type 3 fimbrial shaft (MrkA) of Klebsiella pneumoniae, but not the fimbrial adhesin (MrkD), facilitates biofilm formation. Infect Immun, 2001. 69(9): p. 5805-12.

178. Brett, P.J., D. Deshazer, and D.E. Woods, Characterization of Burkholderia pseudomallei and Burkholderia pseudomallei-like strains. Epidemiol Infect, 1997. 118(2): p. 137-48.

179. Zerbino, D.R. and E. Birney, Velvet: algorithms for de novo short read assembly using de Bruijn graphs. Genome research, 2008. 18(5): p. 8219.

180. Altschul, S.F., et al., Gapped BLAST and PSI-BLAST: a new generation of protein database search programs. Nucleic acids research, 1997. 25(17): p. $3389-402$.

181. Scott, J.B., Genetic characterization of the K2 serotype capsule of Klebsiella pneumoniae ATCC 43816 and the development of a bioluminescent strain, in Program in Oral Biology. 2013, University of Louisville: Louisville.

182. Campos, M.A., et al., Capsule polysaccharide mediates bacterial resistance to antimicrobial peptides. Infect Immun, 2004. 72(12): p. 710714. 
183. Bitter, T. and H.M. Muir, A modified uronic acid carbazole reaction. Analytical biochemistry, 1962. 4: p. 330-4.

184. Donnenberg, M.S. and J.B. Kaper, Construction of an eae deletion mutant of enteropathogenic Escherichia coli by using a positive-selection suicide vector. Infect Immun, 1991. 59(12): p. 4310-7.

185. Simon, R., U. Priefer, and A. Pühler, A broad range mobilization system for in vivo genetic engineering: transposon mutagenesis in gram-negative bacteria. Bio/Technology, 1983. 1: p. 784-791.

186. Struve, C. and K.A. Krogfelt, Role of capsule in Klebsiella pneumoniae virulence: lack of correlation between in vitro and in vivo studies. FEMS microbiology letters, 2003. 218(1): p. 149-54.

187. Lawrenz, M.B., et al., Intubation-mediated Intratracheal (IMIT) Instillation: A Noninvasive, Lung-specific Delivery System. 2014(93): p. e52261.

188. Bagley, S.T., Habitat association of Klebsiella species. Infect Control, 1985. 6(2): p. 52-8.

189. Kesteman, A.S., et al., Emergence of resistant Klebsiella pneumoniae in the intestinal tract during successful treatment of Klebsiella pneumoniae lung infection in rats. Antimicrobial agents and chemotherapy, 2010. 54(7): p. 2960-4.

190. Highsmith, A.K. and W.R. Jarvis, Klebsiella pneumoniae: selected virulence factors that contribute to pathogenicity. Infect Control, 1985. 6(2): p. 75-7. 
191. Chung, D.R., et al., High prevalence of multidrug-resistant nonfermenters in hospital-acquired pneumonia in Asia. American journal of respiratory and critical care medicine, 2011. 184(12): p. 1409-17.

192. Yu, V.L., et al., Virulence characteristics of Klebsiella and clinical manifestations of K. pneumoniae bloodstream infections. Emerging infectious diseases, 2007. 13(7): p. 986-93.

193. Marrie, T.J., H. Durant, and L. Yates, Community-acquired pneumonia requiring hospitalization: 5-year prospective study. Reviews of infectious diseases, 1989. 11(4): p. 586-99.

194. Fang, G.D., et al., New and emerging etiologies for community-acquired pneumonia with implications for therapy. A prospective multicenter study of 359 cases. Medicine, 1990. 69(5): p. 307-16.

195. Marston, B.J., et al., Incidence of community-acquired pneumonia requiring hospitalization. Results of a population-based active surveillance Study in Ohio. The Community-Based Pneumonia Incidence Study Group. Archives of internal medicine, 1997. 157(15): p. 1709-18.

196. Hidron, A.I., et al., NHSN annual update: antimicrobial-resistant pathogens associated with healthcare-associated infections: annual summary of data reported to the National Healthcare Safety Network at the Centers for Disease Control and Prevention, 2006-2007. Infection control and hospital epidemiology : the official journal of the Society of Hospital Epidemiologists of America, 2008. 29(11): p. 996-1011. 
197. CDC, Guidance for control of infections with carbapenem-resistant or carbapenemase-producing Enterobacteriaceae in acute care facilities. MMWR. Morbidity and mortality weekly report, 2009. 58(10): p. 256-60.

198. Doyle, T. and R. Sanderson, Survey of hospital microbiology laboratories regarding Klebsiella pneumoniae carbapenemase (KPC)-producing organisms. Epi Update: Florida Department of Health, 2008: p. 9-12.

199. Marquez, P. and D. Terashita, Carbapenem-resistant Klebsiella pneumoniae (CRKP) surveillance Los Angeles County, June-December 2010. Special Studies Report: LA County Public Health, 2010: p. 25-26.

200. Lavender, H.F., J.R. Jagnow, and S. Clegg, Biofilm formation in vitro and virulence in vivo of mutants of Klebsiella pneumoniae. Infection and immunity, 2004. 72(8): p. 4888-90.

201. Padilla, E., et al., Klebsiella pneumoniae AcrAB efflux pump contributes to antimicrobial resistance and virulence. Antimicrobial agents and chemotherapy, 2010. 54(1): p. 177-83.

202. March, C., et al., Klebsiella pneumoniae outer membrane protein A is required to prevent the activation of airway epithelial cells. The Journal of biological chemistry, 2011. 286(12): p. 9956-67.

203. March, C., et al., Role of bacterial surface structures on the interaction of Klebsiella pneumoniae with phagocytes. PLoS ONE, 2013. 8(2): p. e56847. 
204. Insua, J.L., et al., Modeling Klebsiella pneumoniae pathogenesis by infection of the wax moth Galleria mellonella. Infection and immunity, 2013. 81(10): p. 3552-65.

205. Wu, K.M., et al., Genome sequencing and comparative analysis of Klebsiella pneumoniae NTUH-K2044, a strain causing liver abscess and meningitis. J Bacteriol, 2009. 191(14): p. 4492-501.

206. Liu, P., et al., Complete genome sequence of Klebsiella pneumoniae subsp. pneumoniae HS11286, a multidrug-resistant strain isolated from human sputum. Journal of bacteriology, 2012. 194(7): p. 1841-2.

207. Shin, S.H., et al., Complete genome sequence of the 2,3-butanediolproducing Klebsiella pneumoniae strain KCTC 2242. Journal of bacteriology, 2012. 194(10): p. 2736-7.

208. Cano, V., et al., Klebsiella pneumoniae triggers a cytotoxic effect on airway epithelial cells. BMC Microbiol, 2009. 9: p. 156.

209. Hsieh, P.F., et al., Lipopolysaccharide 01 antigen contributes to the virulence in Klebsiella pneumoniae causing pyogenic liver abscess. PLoS ONE, 2012. 7(3): p. e33155.

210. Liao, C.H., et al., Klebsiella pneumoniae bacteremia and capsular serotypes, Taiwan. Emerging infectious diseases, 2011. 17(6): p. 1113-5.

211. Pan, Y.J., et al., Capsular polysaccharide synthesis regions in Klebsiella pneumoniae serotype K57 and a new capsular serotype. J Clin Microbiol, 2008. 46(7): p. 2231-40. 
212. Srinivasan, V.B., et al., Role of the two component signal transduction system CpxAR in conferring cefepime and chloramphenicol resistance in Klebsiella pneumoniae NTUH-K2044. PLoS ONE, 2012. 7(4): p. e33777.

213. Keynan, Y. and E. Rubinstein, The changing face of Klebsiella pneumoniae infections in the community. Int J Antimicrob Agents, 2007. 30(5): p. 385-9.

214. Brisse, S., et al., Virulent clones of Klebsiella pneumoniae: identification and evolutionary scenario based on genomic and phenotypic characterization. PLoS One, 2009. 4(3): p. e4982.

215. Fouts, D.E., et al., Complete genome sequence of the N2-fixing broad host range endophyte Klebsiella pneumoniae 342 and virulence predictions verified in mice. PLoS Genet, 2008. 4(7): p. e1000141.

216. Fumagalli, O., et al., $\mathrm{N}$-glycosylated proteins are involved in efficient internalization of Klebsiella pneumoniae by cultured human epithelial cells. Infection and immunity, 1997. 65(11): p. 4445-51.

217. Oelschlaeger, T.A. and B.D. Tall, Invasion of cultured human epithelial cells by Klebsiella pneumoniae isolated from the urinary tract. Infection and immunity, 1997. 65(7): p. 2950-8.

218. Sahly, H., et al., Capsule impedes adhesion to and invasion of epithelial cells by Klebsiella pneumoniae. Infection and immunity, 2000. 68(12): $p$. $6744-9$. 
219. Pan, P.C., et al., Mutation in fucose synthesis gene of Klebsiella pneumoniae affects capsule composition and virulence in mice. Experimental biology and medicine, 2011. 236(2): p. 219-26.

220. Pan, Y.J., et al., Use of a Dictyostelium model for isolation of genetic loci associated with phagocytosis and virulence in Klebsiella pneumoniae. Infection and immunity, 2011. 79(3): p. 997-1006.

221. Warawa, J.M., et al., Bioluminescent diagnostic imaging to characterize altered respiratory tract colonization by the Burkholderia pseudomallei capsule mutant. Frontiers in microbiology, 2011. 2: p. 133.

222. Yadav, V., et al., Induction \& resolution of lobar pneumonia following intranasal instillation with Klebsiella pneumoniae in mice. The Indian journal of medical research, 2003. 118: p. 47-52.

223. Rock, C., et al., Frequency of Klebsiella pneumoniae carbapenemase (KPC)-producing and non-KPC-producing Klebsiella species contamination of healthcare workers and the environment. Infect Control Hosp Epidemiol, 2014. 35(4): p. 426-9.

224. Ahmad, T.A., et al., Development of immunization trials against Klebsiella pneumoniae. Vaccine, 2012. 30(14): p. 2411-20.

225. Gupta, A., Hospital-acquired infections in the neonatal intensive care unit-Klebsiella pneumoniae. Semin Perinatol, 2002. 26(5): p. 340-5.

226. Matsen, J.M., The sources of hospital infection. Medicine (Baltimore), 1973. 52(4): p. 271-7. 
227. Jarvis, W.R., et al., The epidemiology of nosocomial infections caused by Klebsiella pneumoniae. Infect Control, 1985. 6(2): p. 68-74.

228. Yankov, I.V. and T.I. Shmilev, Ventilator-associated pneumonias in children (I)--diagnostic criteria, etiology and pathogenesis. Folia Med (Plovdiv), 2012. 54(1): p. 5-11.

229. Doern, G.V., Trends in antimicrobial susceptibility of bacterial pathogens of the respiratory tract. Am J Med, 1995. 99(6b): p. 3s-7s.

230. Jay, S.J., Nosocomial pneumonia. The challenge of a changing clinical spectrum. Postgrad Med, 1983. 74(2): p. 221-5, 228-9, 232-5.

231. Carpenter, J.L., Klebsiella pulmonary infections: occurrence at one medical center and review. Rev Infect Dis, 1990. 12(4): p. 672-82.

232. Karaolis, D.K., et al., Cyclic di-GMP stimulates protective innate immunity in bacterial pneumonia. Infect Immun, 2007. 75(10): p. 4942-50.

233. Romero, E.D., et al., Prevalence of clinical isolates of Escherichia coli and Klebsiella spp. producing multiple extended-spectrum beta-lactamases. Diagn Microbiol Infect Dis, 2007. 59(4): p. 433-7.

234. Nishi, T. and K. Tsuchiya, Therapeutic effects of cefotiam and cefazolin on experimental pneumonia caused by Klebsiella pneumoniae DT-S in mice. Antimicrob Agents Chemother, 1980. 18(4): p. 549-56.

235. Hervas, J.A., et al., Neonatal sepsis and meningitis in Mallorca, Spain, 1977-1991. Clin Infect Dis, 1993. 16(5): p. 719-24.

236. Graybill, J.R., et al., Nosocomial pneumonia. A continuing major problem. Am Rev Respir Dis, 1973. 108(5): p. 1130-40. 
237. Leowski, J., Mortality from acute respiratory infections in children under 5 years of age: global estimates. World Health Stat Q, 1986. 39(2): p. 13844.

238. Arnold, R.S., et al., Emergence of Klebsiella pneumoniae carbapenemase-producing bacteria. South Med J, 2011. 104(1): p. 40-5.

239. Rapp, R.P. and C. Urban, Klebsiella pneumoniae carbapenemases in Enterobacteriaceae: history, evolution, and microbiology concerns. Pharmacotherapy, 2012. 32(5): p. 399-407.

240. da Silva, R.M., J. Traebert, and D. Galato, Klebsiella pneumoniae carbapenemase (KPC)-producing Klebsiella pneumoniae: a review of epidemiological and clinical aspects. Expert Opin Biol Ther, 2012. 12(6): p. 663-71.

241. Chong, Y., Y. Ito, and T. Kamimura, Genetic evolution and clinical impact in extended-spectrum beta-lactamase-producing Escherichia coli and Klebsiella pneumoniae. Infect Genet Evol, 2011. 11(7): p. 1499-504.

242. Nordmann, P., G. Cuzon, and T. Naas, The real threat of Klebsiella pneumoniae carbapenemase-producing bacteria. Lancet Infect Dis, 2009. 9(4): p. 228-36.

243. Massoud, T.F. and S.S. Gambhir, Molecular imaging in living subjects: seeing fundamental biological processes in a new light. Genes Dev, 2003. 17(5): p. $545-80$.

244. Contag, C.H. and M.H. Bachmann, Advances in in vivo bioluminescence imaging of gene expression. Annu Rev Biomed Eng, 2002. 4: p. 235-60. 
245. Close, D.M., et al., In vivo bioluminescent imaging (BLI): noninvasive visualization and interrogation of biological processes in living animals. Sensors (Basel), 2011. 11(1): p. 180-206.

246. Sun, Y., et al., Development of Bioluminescent Bioreporters for In Vitro and In Vivo Tracking of Yersinia pestis. PLoS ONE, 2012. 7(10): p. e47123.

247. Gonzalez, R.J., et al., Bioluminescence imaging to track bacterial dissemination of Yersinia pestis using different routes of infection in mice. BMC Microbiology, 2012. 12(1): p. 1-12.

248. Trcek, J., K. Berschl, and K. Trulzsch, In vivo analysis of Yersinia enterocolitica infection using luxCDABE. FEMS Microbiol Lett, 2010. 307(2): p. 201-6.

249. Mott, T., et al., Monitoring Therapeutic Treatments against Burkholderia Infections Using Imaging Techniques. Pathogens, 2013. 2(2): p. 383.

250. Slate, A.R., et al., Efficacy of enrofloxacin in a mouse model of sepsis. J Am Assoc Lab Anim Sci, 2014. 53(4): p. 381-6.

251. Sha, J., et al., A non-invasive in vivo imaging system to study dissemination of bioluminescent Yersinia pestis CO92 in a mouse model of pneumonic plague. Microb Pathog, 2013. 55: p. 39-50.

252. Zhang, T., S.-Y. Li, and E.L. Nuermberger, Autoluminescent Mycobacterium tuberculosis for Rapid, Real-Time, Non-Invasive Assessment of Drug and Vaccine Efficacy. PLoS ONE, 2012. 7(1): p. e29774. 
253. Francis, K.P., et al., Visualizing pneumococcal infections in the lungs of live mice using bioluminescent Streptococcus pneumoniae transformed with a novel gram-positive lux transposon. Infect Immun, 2001. 69(5): p. $3350-8$.

254. Colardyn, F. and K.L. Faulkner, Intravenous meropenem versus imipenem/cilastatin in the treatment of serious bacterial infections in hospitalized patients. Meropenem Serious Infection Study Group. J Antimicrob Chemother, 1996. 38(3): p. 523-37.

255. Mouton, Y.J. and C. Beuscart, Empirical monotherapy with meropenem in serious bacterial infections. Meropenem Study Group. J Antimicrob Chemother, 1995. 36 Suppl A: p. 145-56.

256. Sieger, B., et al., Empiric treatment of hospital-acquired lower respiratory tract infections with meropenem or ceftazidime with tobramycin: a randomized study. Meropenem Lower Respiratory Infection Group. Crit Care Med, 1997. 25(10): p. 1663-70.

257. Daikos, G.L. and A. Markogiannakis, Carbapenemase-producing Klebsiella pneumoniae: (when) might we still consider treating with carbapenems? Clin Microbiol Infect, 2011. 17(8): p. 1135-41.

258. Tumbarello, M., et al., Predictors of mortality in bloodstream infections caused by Klebsiella pneumoniae carbapenemase-producing $K$. pneumoniae: importance of combination therapy. Clin Infect Dis, 2012. 55(7): p. 943-50. 
259. Robilotti, E. and S. Deresinski, Carbapenemase-producing Klebsiella pneumoniae. F1000Prime Rep, 2014. 6: p. 80.

260. Sarubbi, F., B. Franzus, and A. Verghese, Comparative activity of meropenem (SM-7338) against major respiratory pathogens and amikacin-resistant nosocomial isolates. Eur J Clin Microbiol Infect Dis, 1992. 11(1): p. 65-8.

261. Edwards, J.R., Meropenem: a microbiological overview. J Antimicrob Chemother, 1995. 36 Suppl A: p. 1-17.

262. Birnbaum, J., et al., Carbapenems, a new class of beta-lactam antibiotics. Discovery and development of imipenem/cilastatin. Am J Med, 1985. 78(6a): p. 3-21.

263. Bax, R.P., et al., The pharmacokinetics of meropenem in volunteers. J Antimicrob Chemother, 1989. 24 Suppl A: p. 311-20.

264. Hutchison, M., et al., A compilation of meropenem tissue distribution data. J Antimicrob Chemother, 1995. 36 Suppl A: p. 43-56.

265. Guidelines for the management of adults with hospital-acquired, ventilatorassociated, and healthcare-associated pneumonia. Am J Respir Crit Care Med, 2005. 171(4): p. 388-416.

266. DeRyke, C.A., et al., Bactericidal activities of meropenem and ertapenem against extended-spectrum-beta-lactamase-producing Escherichia coli and Klebsiella pneumoniae in a neutropenic mouse thigh model. Antimicrob Agents Chemother, 2007. 51(4): p. 1481-6. 
267. Salloum, N.A., et al., Assessment of combination therapy in BALB/c mice injected with carbapenem-resistant Enterobacteriaceae strains. Front Microbiol, 2015. 6: p. 999.

268. Lawrenz, M.B., et al., Development and evaluation of murine lung-specific disease models for Pseudomonas aeruginosa applicable to therapeutic testing. Pathog Dis, 2015. 73(5).

269. Shu, H.Y., et al., Genetic diversity of capsular polysaccharide biosynthesis in Klebsiella pneumoniae clinical isolates. Microbiology, 2009. 155(Pt 12): p. $4170-83$.

270. Flores-Mireles, A.L., et al., Urinary tract infections: epidemiology, mechanisms of infection and treatment options. Nat Rev Microbiol, 2015. 13(5): p. 269-84.

271. Siu, L.K., et al., Klebsiella pneumoniae liver abscess: a new invasive syndrome. Lancet Infect Dis, 2012. 12(11): p. 881-7.

272. Girometti, N., et al., Klebsiella pneumoniae bloodstream infection: epidemiology and impact of inappropriate empirical therapy. Medicine (Baltimore), 2014. 93(17): p. 298-309.

273. Craig, A., et al., Neutrophil recruitment to the lungs during bacterial pneumonia. Infect Immun, 2009. 77(2): p. 568-75.

274. Harada, A., et al., Essential involvement of interleukin-8 (IL-8) in acute inflammation. J Leukoc Biol, 1994. 56(5): p. 559-64. 
275. Mikerov, A.N., et al., Sex differences in the impact of ozone on survival and alveolar macrophage function of mice after Klebsiella pneumoniae infection. Respiratory Research, 2008. 9(1): p. 24-24.

276. Liu, T., et al., Differences in expression of toll-like receptors and their reactivities in dendritic cells in BALB/C and C57BL/6 mice. Infect Immun, 2002. 70(12): p. 6638-45.

277. Chen, X., J.J. Oppenheim, and O.M. Howard, BALB/c mice have more $C D 4+C D 25+T$ regulatory cells and show greater susceptibility to suppression of their CD4+CD25- responder T cells than C57BL/6 mice. J Leukoc Biol, 2005. 78(1): p. 114-21.

278. Watanabe, $\mathrm{H}$., et al., Innate immune response in Th1- and Th2-dominant mouse strains. Shock, 2004. 22(5): p. 460-6.

279. Karupiah, G., Type 1 and type 2 cytokines in antiviral defense. Vet Immunol Immunopathol, 1998. 63(1-2): p. 105-9.

280. Okano, M., et al., Strain-dependent induction of allergic rhinitis without adjuvant in mice. Allergy, 1999. 54(6): p. 593-601.

281. Autenrieth, I.B., et al., Role of T-helper-cell subtypes and cytokines in immunity to Yersinia enterocolitica in susceptible and resistant strains of mice. Contrib Microbiol Immunol, 1995. 13: p. 203-6.

282. Autenrieth, I.B., et al., Immune responses to Yersinia enterocolitica in susceptible BALB/c and resistant C57BL/6 mice: an essential role for gamma interferon. Infect Immun, 1994. 62(6): p. 2590-9. 
283. Ellermann-Eriksen, S., J. Justesen, and S.C. Mogensen, Genetically determined difference in the antiviral action of alpha/beta interferon in cells from mice resistant or susceptible to herpes simplex virus type 2. J Gen Virol, 1986. 67 ( Pt 9): p. 1859-66.

284. Pepose, J.S. and J.A. Whittum-Hudson, An immunogenetic analysis of resistance to herpes simplex virus retinitis in inbred strains of mice. Invest Ophthalmol Vis Sci, 1987. 28(9): p. 1549-52.

285. Reiner, S.L. and R.M. Locksley, The regulation of immunity to Leishmania major. Annu Rev Immunol, 1995. 13: p. 151-77.

286. Kirchner, H., et al., Immunological Studies of HSV-Infection of Resistant and Susceptible Inbred Strains of Mice. Zeitschrift für Immunitätsforschung: Immunobiology, 1978. 154(2): p. 147-154.

287. Price, C.T. and Y. Abu Kwaik, The transcriptome of Legionella pneumophila-infected human monocyte-derived macrophages. PLoS One, 2014. 9(12): p. e114914.

288. Kadioglu, A., et al., The role of Streptococcus pneumoniae virulence factors in host respiratory colonization and disease. Nat Rev Micro, 2008. 6(4): p. 288-301.

289. Kostyanev, T.S. and L.P. Sechanova, Virulence factors and mechanisms of antibiotic resistance of haemophilus influenzae. Folia Med (Plovdiv), 2012. 54(1): p. 19-23.

290. King, P., Haemophilus influenzae and the lung (Haemophilus and the lung). Clinical and Translational Medicine, 2012. 1(1): p. 1-9. 
291. Powers, M.E. and J.B. Wardenburg, Igniting the Fire: Staphylococcus aureus Virulence Factors in the Pathogenesis of Sepsis. PLoS Pathogens, 2014. 10(2): p. e1003871.

292. Zecconi, A. and F. Scali, Staphylococcus aureus virulence factors in evasion from innate immune defenses in human and animal diseases. Immunol Lett, 2013. 150(1-2): p. 12-22.

293. Revell, P.A. and V.L. Miller, Yersinia virulence: more than a plasmid. FEMS Microbiol Lett, 2001. 205(2): p. 159-64.

294. van Opijnen, T., K.L. Bodi, and A. Camilli, Tn-seq; high-throughput parallel sequencing for fitness and genetic interaction studies in microorganisms. Nature methods, 2009. 6(10): p. 767-772.

295. van Opijnen, T. and A. Camilli, Transposon insertion sequencing: a new tool for systems-level analysis of microorganisms. Nat Rev Micro, 2013. 11(7): p. 435-442.

296. Gutierrez, M.G., D.R. Yoder-Himes, and J.M. Warawa, Comprehensive identification of virulence factors required for respiratory melioidosis using Tn-seq mutagenesis. Front Cell Infect Microbiol, 2015. 5: p. 78.

297. Bonemann, G., A. Pietrosiuk, and A. Mogk, Tubules and donuts: a type VI secretion story. Mol Microbiol, 2010. 76(4): p. 815-21.

298. Coulthurst, S.J., The Type VI secretion system - a widespread and versatile cell targeting system. Res Microbiol, 2013. 164(6): p. 640-54. 
299. Zhang, L., et al., TssB is essential for virulence and required for type VI secretion system in Ralstonia solanacearum. Microb Pathog, 2014. 74: p. $1-7$.

300. de Pace, F., et al., The type VI secretion system plays a role in type 1 fimbria expression and pathogenesis of an avian pathogenic Escherichia coli strain. Infect Immun, 2010. 78(12): p. 4990-8.

301. Miyata, S.T., et al., Vibrio cholerae requires the type VI secretion system virulence factor VasX to kill Dictyostelium discoideum. Infect Immun, 2011. 79(7): p. 2941-9.

302. Weyrich, L.S., et al., A Type VI secretion system encoding locus is required for Bordetella bronchiseptica immunomodulation and persistence in vivo. PLoS One, 2012. 7(10): p. e45892.

303. Sarris, P.F., et al., Distribution of the putative type VI secretion system core genes in Klebsiella spp. Infect Genet Evol, 2011. 11(1): p. 157-66.

304. Broberg, C.A., et al., Complete Genome Sequence of Klebsiella pneumoniae Strain ATCC 43816 KPPR1, a Rifampin-Resistant Mutant Commonly Used in Animal, Genetic, and Molecular Biology Studies. Genome Announc, 2014. 2(5).

305. Basler, M., B.T. Ho, and J.J. Mekalanos, Tit-for-tat: type VI secretion system counterattack during bacterial cell-cell interactions. Cell, 2013. 152(4): p. 884-94. 
306. Gutierrez, M.G., T.L. Pfeffer, and J.M. Warawa, Type 3 secretion system cluster 3 is a critical virulence determinant for lung-specific melioidosis. PLoS Negl Trop Dis, 2015. 9(1): p. e34 


\section{CURRICULUM VITAE}

Ramy Fodah

1830 S $3^{\text {rd }}$ ST., Louisville, KY 40208

$216-258-1492$

\section{EDUCATION}

- Ph.D., Microbiology and Immunology, University of Louisville, KY 2016

- M.S., Microbiology and Immunology, University of Louisville, KY 2012

- B.S., Clinical Laboratory Sciences, King Saudi University, KSA 2004

\section{RESEARCH EXPERIENCE}

Graduate Research Assistant

2011 - Present

Department of Microbiology and Immunology, University of Louisville Dissertation Chair: Jonathan M. Warawa, Ph.D.

Project: Identifying virulence factors required by Klebsiella pneumoniae to establish a respiratory infection using lung-specific mouse disease model.

- Planned, carried out and analyzed experimental results to determine attenuation level, differential phenotype and trafficking pattern associated with distinct $K$. pneumoniae mutant strains in in vitro and in vivo assays. 
- Followed safety precautions and handling of K. pneumoniae in BSL-2 laboratory space in compliance with UofL OSHA and DEHS guidelines.

- Resolved technical problems associated with scientific protocols, procedures, and equipment.

- Prepared study protocols, spreadsheets, charts, graphs, presentations and manuscripts to communicate experimental work flow and results to both scientists and the general public.

\section{TECHNICAL RESEARCH EXPERTISE}

Mouse model of respiratory disease

Polyacrylamide gel electrophoresis

Cell culture

Western blotting

Molecular cloning

Bioinformatics

Sequencing

Statistics

Primer design

Purification
In vivo imaging

Microbiology

DNA extraction

Scientific Writing

Sequence analysis

Mutagenesis

GraphPad Prism

Microscopy
Agarose gel electrophoresis

Polymerase chain reaction

High-throughput screening

Molecular biology

Bacterial transformation

Animal models

Enzyme Assays

Experimental design

Antibodies generation

Spectrophotometry 


\section{WORK EXPERIENCE}

- Teaching assistant

$2008-2009$

King Saud bin Abdulaziz University for health Sciences (KSAU-HS), Saudi Arabia, Riyadh.

- Medical Technologist II $2006-2008$ National Guard Health Affairs (NGHA), Saudi Arabia, Riyadh Department of Pathology and Laboratory Medicine, Microbiology section: Performed Medical Technologist II (MedTech-II) duties which include sample receiving, processing, identification of microbial pathogens in clinical samples, and antibiotic susceptibility testing.

\section{PUBLICATIONS}

Fodah, R. A., Scott, J. B., Tam, H. H., Yan, P., Pfeffer, T. L., Bundschuh, R., \& Warawa, J. M. (2014). Correlation of Klebsiella pneumoniae comparative genetic analyses with virulence profiles in a murine respiratory disease model. PLoS One, 9(9), e107394. doi:10.1371/journal.pone.0107394

Lawrenz, M. B., Fodah, R. A., Gutierrez, M. G., \& Warawa, J. (2014). Intubationmediated Intratracheal (IMIT) Instillation: A Noninvasive, Lung-specific Delivery System. (93), e52261. doi:doi:10.3791/52261

Mo, Y., Chen, J., Humphrey, D. M., Jr., Fodah, R. A., Warawa, J. M., \& Hoyle, G. W. (2015). Abnormal epithelial structure and chronic lung inflammation after repair of chlorine-induced airway injury. Am J Physiol Lung Cell Mol Physiol, 308(2), L168-178. doi:10.1152/ajplung.00226.2014 


\section{PRESENTATIONS}

Fodah RA, Pfeffer TL, Warawa JM. The development of bioluminescent Klebsiella pneumoniae to study pulmonary disease in a murine infection model. Research!Louisville, 2013. Poster presentation.

Fodah RA, Scott JB, Warawa JM. Klebsiella pneumoniae is capable of intracellular persistence in phagocytes. ICAAC conference at Washington, DC, 2014. Poster presentation.

Fodah RA, Scott JB, Warawa JM. Improved monitoring of bacterial viability using bioluminescent Klebsiella pneumoniae. Research!Louisville, 2015. Poster presentation.

\section{HONORS AND AWARDS}

- Norton Healthcare Medical student award for Research!Louisville, 2013 poster presentation. 